\title{
Kritik und Revision des Qualia-Begriffs
}

In meiner Analyse des in der Forschungsliteratur verwendeten Qualia-Begriffs werde ich erstens aufzeigen, dass aufgrund einer viel zu engen Auffassung von Qualia das Desiderat phänomenaler Adäquatheit oft verfehlt wird und deshalb das hard problem nicht gebührend erfasst wird. In diesem Zusammenhang soll eine, mit engen Qualia-Begriffen einhergehende Tendenz sichtbar gemacht werden, die darin besteht, auf der Basis einer Kritik von bestimmten, angeblichen Qualia-Eigenschaften, die jedoch nicht notwendigerweise Eigenschaften von Qualia sind, phänomenales Erleben in toto anzuzweifeln oder zu leugnen (Abschnitt 8.1).

In einem zweiten Schritt (Abschnitt 8.2) werde ich einen phänomenal adäquaten Qualia-Begriff ausarbeiten, welcher der Ubiquität, Vielfalt, Komplexität und Feinkörnigkeit phänomenalen Erlebens gerechter werden soll und begründen, warum bestimmte emotionale und sogar kognitiv-gedankliche sowie intentionale Zustände auch als Qualia verstanden werden sollten.

\subsection{Phänomenale Inadäquatheit des herkömmlichen Qualia-Begriffs}

Ein phänomenal adäquater Qualia-Begriff stellt den Ausgangs- und Angelpunkt eines Lösungsversuches des hard problem dar, der auch hinreichend dessen Explanandum berücksichtigen will. ${ }^{1}$ Trotzdem gibt es keinen klaren Konsens, was Qualia sein sollen; ${ }^{2}$ auch nicht hinsichtlich dessen, was (noch) als Quale gilt, und was nicht. Infolgedessen kommen Heilinger und Jung zum Schluss, dass die Debatte über Qualia unüberschaubar geworden ist. ${ }^{3}$

1 Dementsprechend stellt Metzinger fest, dass Qualia das »Kardinalproblem« der Philosophie des Geistes darstellen. Vgl. Metzinger, Thomas: Subjekt und Selbstmodell. Die Perspektivität phänomenalen Bewusstseins vor dem Hintergrund einer naturalistischen Theorie mentaler Repräsentation, Paderborn: Mentis 1999, S. 89. Vgl. dazu auch Stubenberg, Leopold: Consciousness and qualia, Amsterdam: John Benjamins 1998, S. 13.

2 Vgl. dazu Crane, Tim: »The origins of qualia«, 200o, Online: http://sas-space.sas.ac.uk/220/1/ The\%20origins\%20of\%2oqualia.pdf [zuletzt geprüft am 24.09.2018], S. 2 und 21.

3 Vgl. Jung, Matthias / Heilinger, Jan-Christoph [Hrsg.]: Funktionen des Erlebens. Neue Perspektiven des qualitativen Bewusstseins. Band 5: Humanprojekt, Berlin: De Gruyter 2009, S. 6. 
Dennoch lässt sich feststellen, dass der in der Forschungsliteratur zumeist verwendete Qualia-Begriff sich auf vornehmlich visuelle Sinneserfahrungen und Wahrnehmungen des eigenen Körpers beschränkt.. ${ }^{4}$ Bei den visuellen Sinneserfahrungen sind Beispiele von homogenen, statischen Farbwahrnehmungen ${ }^{5}$ bzw. Farb-Qualia ${ }^{6}$ besonders dominant und bei den Körperwahrnehmungen wird insbesondere das Standardbeispiel $>$ Schmerz ${ }^{7}$ herangezogen. Der Bedeutungsumfang von Qualia wird demnach oft eingeschränkt auf monosensuale Schemata von Erleben. ${ }^{8}$ Aber ein solch enger, statischer Qualia-Begriff mit welchem man sich zumeist nur auf wenige Beispiele einfachster phänomenaler Gehalte bezieht, vernachlässigt einerseits den diachronen Charakter phänomenalen Erlebens und andererseits die Vielfalt und Reichhaltigkeit heterogener bzw. komplexer Erlebnis-Nuancen. ${ }^{9}$

Sonach stellt die, mit einem monadisch-statischen Qualia-Begriff zusammenhängende, »substantivistische Redeweise ${ }^{10}$ von Qualia (im Sinn von

4 Vgl. dazu auch Staudacher, Alexander: Phänomenales Bewusstsein als Problem für den Materialismus, Berlin: De Gruyter 2002, S. 5. Eher selten werden auditiven Formen von Sinnes-Qualia ausführlich als Beispiele geschildert. So z.B. bei Chalmers, David J.: The conscious mind. In search of a fundamental theory, New York: Oxford University Press 1996, S. 7. Ebenfalls werden bei körperlichen Empfindungen seltener bspw. deren taktile Formen berücksichtigt. Eine Ausnahme bildet bspw. a.a.O., S. 8.

5 Vgl. z.B. Lycan, William: »Representational Theories of Consciousness «, in: The Stanford Encyclopedia of Philosophy, 2008, Online: http://plato.stanford.edu/archives/fall20o8/ entries/consciousness-representational/ [zuletzt geprüft am 28.09.2018], Abschnitt 2.3. Vgl. ebenfalls Lycan, William G. [Hrsg.]: Mind and cognition. An anthology, Malden, Massachusetts: Blackwell 1999, S. 450.

6 Vgl. Chalmers, David J.: The conscious mind. In search of a fundamental theory, New York: Oxford University Press 1996, S. 6. Vgl. dazu auch Slaby, Jan: Gefühl und Weltbezug. Die menschliche Affektivität im Kontext einer neo-existentialistischen Konzeption von Personalität, Paderborn: Mentis 2008, S. 316.

$7 \quad$ Vgl. Z.B. Pitt, David: »The Phenomenology of Cognition or what is it Like to Think that P? «, Philosophy and Phenomenological Research, [2004] Vol. 69, No. 1, S. 3o. Oder: Metzinger, Thomas [Hrsg.]: Grundkurs Philosophie des Geistes. Band 1: Phänomenales Bewusstsein, Paderborn: Mentis 2007, S. 203. Oder: Staudacher, Alexander: Phänomenales Bewusstsein als Problem für den Materialismus, Berlin: De Gruyter 2002, S. 339. Oder: Lanz, Peter: Das phänomenale Bewusstsein. Eine Verteidigung, Frankfurt am Main: Klostermann 1996, S. 201 und 228.

8 Lorenz, Kuno: »Qualia«, in: Enzyklopädie Philosophie und Wissenschaftstheorie. Band 3: $P$-So, Mittelstrass, Jürgen [Hrsg.], Stuttgart: Metzler 2004, S. 426-427.

9 Vgl. Jung, Matthias / Heilinger, Jan-Christoph [Hrsg.]: Funktionen des Erlebens. Neue Perspektiven des qualitativen Bewusstseins. Band 5: Humanprojekt, Berlin: De Gruyter 20o9, S. 9-11. Vgl. dazu auch Chalmers, David J.: The conscious mind. In search of a fundamental theory, New York: Oxford University Press 1996, S. 7 .

10 Jung, Matthias / Heilinger, Jan-Christoph [Hrsg.]: Funktionen des Erlebens. Neue Perspektiven des qualitativen Bewusstseins. Band 5: Humanprojekt, Berlin: De Gruyter 20og, S. 9. 
>diskreten< mentalen Entitäten bzw. Zuständen wie einer >Rotimpression< oder >einem Schmerz $<$ ) eine folgenreiche Vorentscheidung dar, die dem phänomenalen Befund des Verlaufscharakters und dem Aspekt der Gesamtheit komplexer Erlebnis-Nuancen nicht im Ansatz gerecht wird." So verwendet beispielsweise Dennett einen Qualia-Begriff, bei dem er von »essentials $«^{12}$ bzw. von »special properties $«^{13}$ wie $»[\ldots]$ subjective tastes, looks, aromas, sounds $[. ..] \ll^{14}$ ausgeht. Auch Metzinger beschreibt eine Auffassung von Qualia nach der diese eine »essentialistische « ${ }^{15}$ Intuition darstellen sollen. Dazu passend, geht Dennett davon aus, dass Befürworter von Qualia glauben würden, dass sie solche >Qualia< für Definitionszwecke isolieren könnten. ${ }^{16}$ An anderer Stelle spricht er von der »Illusion der Homogenität« (»doctrinal illusion of >homogeneity «), ${ }^{17}$ die das Qualia-Verständnis der philosophischen Tradition charakterisieren soll, was ebenfalls diesem sehr engen, statischen und monosensualen Verständnis ${ }^{18}$ von Qualia entspricht. Mit diesem eigenartigen und monadischen Qualia-Begriff steht er nicht alleine da. Nach Van Gulick geht man generell beim traditionellen Qualia-Verständnis davon aus, dass diese intrinsisch, privat, unaussprechlich und wie Monaden $^{19} \mathrm{zu}$

\footnotetext{
11 Vgl. dazu a.a.O., S. 10.

12 Dennett, Daniel C.: »Quining Qualia«, in: Philosophy of mind. Classical and contemporary readings, Chalmers, David J. [Hrsg.], New York: Oxford University Press [zuerst 1988]/2002, S. 228.

13 Ebd.

14 Ebd.

15 Metzinger, Thomas: Subjekt und Selbstmodell. Die Perspektivität phänomenalen Bewusstseins vor dem Hintergrund einer naturalistischen Theorie mentaler Repräsentation, Paderborn: Mentis 1999, S. 36.

16 Vgl. Dennett, Daniel C.: »Quining Qualia«, in: Philosophy of mind. Classical and contemporary readings, Chalmers, David J. [Hrsg.], New York: Oxford University Press [zuerst 1988]/2002, S. 228.

17 A.a.O., S. 243. Zur Konzeption von Qualia als »homogene« Entitäten vgl. auch Metzinger, Thomas: Subjekt und Selbstmodell. Die Perspektivität phänomenalen Bewusstseins vor dem Hintergrund einer naturalistischen Theorie mentaler Repräsentation, Paderborn: Mentis 1999, S. 36.

18 Zum monosensualen Verständnis von Qualia vgl.: Lorenz, Kuno: Artikel »Qualia« in: Enzyklopädie Philosophie und Wissenschaftstheorie, [Hrsg.: Mittelstrass, Jürgen], Stuttgart / Weimar: J. B. Metzler Verlag 2004, Band 3, S. 426-427.

19 Zur monadischen Konzeption von Qualia vgl. auch Lycan, William: »Representational Theories of Consciousness«, in: The Stanford Encyclopedia of Philosophy, 2008, Online: http://plato.stanford.edu/archives/fall20o8/entries/consciousness-representational/ [zuletzt geprüft am 28.o9.2018], Abschnitt 1.o. Vgl. ebenfalls Metzinger, Thomas: Subjekt und Selbstmodell. Die Perspektivität phänomenalen Bewusstseins vor dem Hintergrund einer naturalistischen Theorie mentaler Repräsentation, Paderborn: Mentis 1999, S. 33.
} 
verstehen seien. ${ }^{20}$ Diesbezüglich ist auch von $»$ Atomen des Bewusstseins $«,{ }^{21}$ einer $»$ Unteilbarkeits-Intuition «, 22 »basale[n] Bewusstseinsatome[n] «, ${ }^{23}$ oder »phänomenale[n] Atome[n] $\ll^{24}$ die Rede; unter einem >Atom< versteht man bei dieser Konzeption ein Quale eines »einfachen phänomenalen Inhalt $[\mathrm{s}] \ll{ }^{25}$ Eine solche Qualia-Konzeption gründet auf problematischen ontologische Entitäten, die Qualia-Gegner ${ }^{26}$ zu Recht als Atomismus ${ }^{27}$ des Bewusstseins

20 Vgl. Van Gulick, Robert: »Consciousness«, in: The Stanford Encyclopedia of Philosophy, 2014, Online: http://plato.stanford.edu/archives/spr2014/entries/consciousness/ [zuletzt geprüft am 17.09.2018], Abschnitt 2.2.

21 Metzinger, Thomas [Hrsg.]: Grundkurs Philosophie des Geistes. Band 1: Phänomenales Bewusstsein, Paderborn: Mentis 2007, S. 58. Metzinger führt jedoch selber auch Gegenargumente zu diesem atomistischen Qualia-Begriff auf: Vgl. dazu Metzinger, Thomas: Subjekt und Selbstmodell. Die Perspektivität phänomenalen Bewusstseins vor dem Hintergrund einer naturalistischen Theorie mentaler Repräsentation, Paderborn: Mentis 1999, S. 36. Zu Metzingers Kritik an »phänomenalen Individuen« vgl. auch Metzinger, Thomas: »Präsentationaler Gehalt«, in: Bewusstsein und Repräsentation, Esken, Frank / Heckmann, Heinz-Dieter [Hrsg.], Paderborn: Mentis 1999, S. 3.

22 Metzinger, Thomas: Subjekt und Selbstmodell. Die Perspektivität phänomenalen Bewusstseins vor dem Hintergrund einer naturalistischen Theorie mentaler Repräsentation, Paderborn: Mentis 1999, S. 36 .

23 Kemmerling, Andreas: »Eine Handvoll Bemerkungen zur begrifflichen Unübersichtlichkeit von >Bewusstsein««, in: Bewusstsein und Repräsentation, Esken, Frank / Heckmann, Heinz-Dieter [Hrsg.], Paderborn: Mentis 1999.

24 Metzinger, Thomas: Subjekt und Selbstmodell. Die Perspektivität phänomenalen Bewusstseins vor dem Hintergrund einer naturalistischen Theorie mentaler Repräsentation, Paderborn: Mentis 1999, S. 36.

25 Metzinger, Thomas [Hrsg.]: Grundkurs Philosophie des Geistes. Band 1: Phänomenales Bewusstsein, Paderborn: Mentis 2007, S. 203. Nach diesem Verständnis sind Qualia die einfachsten Erscheinungsformen des phänomenalen Erlebens. Vgl. a.a.O., S. 57. Weitere Beispiele einer atomistisch-monosensualen Verwendungsweise des Qualia-Begriffs finden sich bei Robinson, William S.: »Qualia realism«, in: A Field Guide to the Philosophy of Mind, SIFA, Società Italiana Filosofia Analitica, 2002, Online: http://host.uniroma3. it/progetti/kant/field/qr.htm [zuletzt geprüft am 25.09.2018], Abschnitt einleitender Erläuterungen.

26 Selbstverständlich kritisieren nicht nur Qualia-Gegner das atomistische Verständnis von Qualia. So ist Fuchs gegen die Idee einer atomistischen Teilbarkeit leiblicher Subjektivität, weil diese nach ihm unteilbar ist. Sind wir bspw. missgestimmt, so betrifft dies das ganze leiblich-subjektive Erleben. Vgl. Fuchs, Thomas: »Hirnwelt oder Lebenswelt? Zur Kritik des Neurokonstruktivismus«, Deutsche Zeitschrift für Philosophie, [2011] Vol. 59, No. 3 , S. 355 .

27 Dennett spricht von einem »Atomismus« (»atomicity to analysis«) welches das QualiaVerständnis der philosophischen Tradition charakterisieren soll. Vgl. Dennett, Daniel C.: »Quining Qualia«, in: Philosophy of mind. Classical and contemporary readings, Chalmers, David J. [Hrsg.], New York: Oxford University Press [zuerst 1988]/2002, S. 243. 
kritisieren. ${ }^{28}$ So zweifelt bspw. Metzinger - auf der Grundlage einer derart verengten Qualia-Konzeption - an der Existenz von Qualia:»[...] gibt es eigentlich $>$ den< einfachen phänomenalen Inhalt? «. ${ }^{29}$

Für Katalin Balog besteht die Gemeinsamkeit bei Ansätzen mit einem »Qualia-Realismus «0 darin, dass Qualia insofern existieren, als es irgendwie ist, ein Erlebnis zu haben - als etwas, das man normalerweise »introspektiv «31 wahrnehmen könne. Die damit verbundene Konzeption eines »introspektiven $\ll^{32}$ Charakters von Qualia wird bspw. von Armstrong als ein >Scannen sscannenden< Teil interpretiert. ${ }^{33}$ So wird der Qualia-Begriff oft auch im

28 Dennett kritisiert ebenfalls jenes atomistische Qualia-Verständnis; scheint diesbezüglich aber zu glauben, damit Qualia widerlegen zu können: »Qualia seem atomic to introspection, unanalyzable simples - the smell of violets, the shade of blue, the sound of an oboe - but this is clearly an effect of something like the resolution of our discernment machinery . Dennett, Daniel C.: »Why and How Does Consciousness Seem the Way it Seems? «, 2015, Online: http://open-mind.net/papers/why-and-how-does-consciousnessseem-the-way-it-seems [zuletzt geprüft am 24.09.2018], S. 8. So kommt er zum Schluss: $»[\ldots]$ color experiences are no more atomic than scale tone experiences [...] «. A.a.O., S. 10.

29 Metzinger, Thomas [Hrsg.]: Grundkurs Philosophie des Geistes. Band 1: Phänomenales Bewusstsein, Paderborn: Mentis 2007, S. 203 [Hervorhebung im Original]. Vgl. ebenfalls a.a.O., S. $57-58$.

30 Balog, Katalin: »Phenomenal Concepts«, in: The Oxford Handbook of Philosophy of Mind, McLaughlin, Brian P. / Beckermann, Ansgar / Walter, Sven [Hrsg.], Oxford: Oxford University Press 2009, S. 293.

31 Ebd. Balog zufolge unterscheiden diese Ansätze jedoch im Hinblick darauf, was es genau heisse, Qualia »introspektiv« wahrzunehmen. Vgl. dazu ebd.

32 Zur Konzeption von Qualia als etwas »Introspektives« vgl. z.B. Feser, Edward: »Qualia. Irreducibly Subjective but not Intrinsic«, Journal of Consciousness Studies, [2001] Vol. 8, No. 8, S. 5. Oder: Lycan, William: »Representational Theories of Consciousness«, in: The Stanford Encyclopedia of Philosophy, 2008, Online: http://plato.stanford.edu/archives/ fall20o8/entries/consciousness-representational/ [zuletzt geprüft am 28.og.2018], Abschnitt 1.o. Oder: Beaton, Michael: »Qualia and Introspection«, Journal of Consciousness Studies, [2009] Vol. 16, No. 5, S. 105. Oder: Churchland, Paul M.: »The Rediscovery of Light«, The Journal of Philosophy, [1996] Vol. 93, No. 5, S. 227.

33 Vgl. Güzeldere, Güven: »Ist Bewusstsein die Wahrnehmung dessen, was im eigenen Geist vorgeht?«, in: Grundkurs Philosophie des Geistes. Band 1: Phänomenales Bewusstsein, Metzinger, Thomas [Hrsg.], Paderborn: Mentis [zuerst 1995, engl.]/2007, S. 362. Vgl. dazu auch Levin, Janet: »Functionalism «, in: The Stanford Encyclopedia of Philosophy, 2013, Online: http://plato.stanford.edu/archives/fall2o13/entries/functionalism/ [zuletzt geprüft am 28.og.2018], Abschnitt 5.3. Demgemäss weist bspw. McDonald darauf hin, dass mit dem Begriff >Introspektion< ein >innerlicher< epistemischer Prozess bezeichnet werde, durch welchen mentale Zustände bewusst würden: »Introspection< is a term used by philosophers to refer to a special method or means by which one comes to know certain of one's own mental states; specifically, one's current conscious states. It derives from the

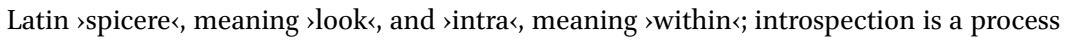


Sinne »intrinsischer « ${ }^{34}$ Erlebnisqualitäten, die nur dem Subjekt zugänglich sind, definiert. ${ }^{35}$ Deshalb bemüht sich Dennett, die angebliche $>$ Intrinsitä̈ ${ }^{36}$ von Qualia mit mehreren >Intuitionspumpen` zu widerlegen. ${ }^{37}$ Er versucht dies beispielsweise mit dem Verweis auf »öffentliche Rotheit«, 38 die als relationale Eigenschaft fälschlicherweise in die Köpfe von Subjekten projiziert werde. ${ }^{39}$ Es handle sich bei ihr jedoch um eine relationale, Eigenschaft, weil »objektive Rotheit ${ }^{40}$ oder objektive Bitterkeit einen ganz bestimmten Effekt auf normale Subjekte habe. Diesen Effekt gelte es von intrinsischen Eigenschaften zu isolieren, denn es genüge nicht, einfach zu behaupten, dass es

of looking inward«. McDonald, Cynthia: »Introspection«, in: The Oxford Handbook of Philosophy of Mind, McLaughlin, Brian P. / Beckermann, Ansgar / Walter, Sven [Hrsg.], Oxford: Oxford University Press 20o9, S. 741.

34 So ist nach Harman das Erleben der Röte eines Apfels eine »intrinsische« Qualität unseres Erlebens: »[...] when you attend to a pain [...] or to your experience of the redness of an apple, you are aware of an intrinsic quality of your experience, where an intrinsic quality is a quality something has in itself, apart from its relations to other things«. Harman, Gilbert: »The intrinsic quality of experience «, in: Philosophy of mind. Critical concepts in philosophy. Volume 4: Consciousness, Crawford, Sean [Hrsg.], London: Routledge \& Kegan Paul [zuerst 1990]/2011, S. 105.

35 Auch nach Dennett besteht eine verbreitete und irrtümliche Ansicht über Erlebnisqualitäten darin, dass diese »intrinsisch« seien. Vgl. Dennett, Daniel C.: »Why and How Does Consciousness Seem the Way it Seems? «, 2015, Online: http://open-mind. net/papers/why-and-how-does-consciousness-seem-the-way-it-seems [zuletzt geprüft am 24.09.2018], S. 4. Zur Konzeption von Qualia als etwas >Intrinsisches« vgl. auch Lorenz, Kuno: »Qualia«, in: Enzyklopädie Philosophie und Wissenschaftstheorie. Band 3: $P$-So, Mittelstrass, Jürgen [Hrsg.], Stuttgart: Metzler 2004, S. 426-427. Vgl. ebenfalls Van Gulick, Robert: »Consciousness«, in: The Stanford Encyclopedia of Philosophy, 2014, Online: http://plato.stanford.edu/archives/spr2o14/entries/consciousness/ [zuletzt geprüft am 17.09.2018], Abschnitt 2.2. Nach Tye wird die Konzeption >intrinsischer Qualia< im Sinne nicht-repräsentationaler Eigenschaften von Vertretern der Sinnesdatentheorie verwendet. Vgl. dazu Tye, Michael: »Qualia«, in: The Stanford Encyclopedia of Philosophy, 2007, Online: http://plato.stanford.edu/archives/fall2007/entries/qualia/ [zuletzt geprüft am 25.09.2018], Abschnitt 1.o. Eine Begründung dafür, warum Qualia intrinsisch sein sollen, findet sich bei Michel, Jan G.: Der qualitative Charakter bewusster Erlebnisse. Physikalismus und phänomenale Eigenschaften in der analytischen Philosophie des Geistes, Paderborn: Mentis 2011, S. 278.

36 Vgl. Dennett, Daniel C.: »Quining Qualia«, in: Philosophy of mind. Classical and contemporary readings, Chalmers, David J. [Hrsg.], New York: Oxford University Press [zuerst 1988]/2002, S. 229.

37 Vgl. dazu a.a.O., S. $235^{-241 .}$

38 A.a.O., S. 241.

39 Vgl. ebd.

40 Ebd. 
»offensichtlich ${ }^{41}$ sei, dass es daneben noch intrinsische Eigenschaften gebe. ${ }^{42}$ Für ihn scheint es jedoch offensichtlich zu sein, dass phänomenales Erleben keine anderen Eigenschaften aufweist, ausser dispositionalen, reaktionserzeugenden Eigenschaften. ${ }^{43}$ Um dies zu untermauern, greift Dennett auf das weiter unten erwähnte Gedankenexperiment der beiden Kaffeetester Chase und Sanborn zurück, ${ }^{44}$ welches, wie ich noch darlegen werde, nur aufzeigt, dass phänomenales Erleben nicht notwendigerweise sunmittelbar<, d.h. unabhängig von kognitiven Prozessen stattfindet (so interagieren auch bei Emotionen kognitive Prozesse mit somatisch-emotionalem Erleben). ${ }^{45}$

Als Beispiel für die angebliche Qualia-Eigenschaft der Intrinsität greift Dennett auch auf Qualia-Konzeptionen mit dem oben erwähnten externalistischen Fehlschluss von bestimmten Formen des Repräsentationalismus zurück: »If challenged to explain the idea of an intrinsic property to a neophyte, many people would hit on the following sort of example: consider Tom's ball; it has many properties, such as its being made of rubber from India, its belonging to Tom [...] and its redness «. ${ }^{46}$ Natürlich fällt es ihm dann leicht, die Oberflächeneigenschaft des Balles (>Rot<) bzw. die angebliche Qualia-Eigenschaft einer »öffentlichen Rotheit«, 47 von der man glaube, sie sei >intrinsisch<, als relationale Eigenschaft zu definieren: »Redness - public redness - is a quintessentially relational property $[\ldots] \ll .48$

Auch Paul Churchland fährt auf dieser phänomenal inadäquaten Argumentationsschiene, indem er sich auf die vermeintlich notwendige Qualia-Eigenschaft der $»$ Introspektion $\aleph^{49}$ beruft, welche er mit deren $»$ Eigenheiten « (»quirks « $)^{50}$ in Verbindung bringt. Interessant ist dabei, dass er die Aufspaltung unseres epistemischen Zuganges zur Welt in >Introspektion $<$ und

\footnotetext{
41 Vgl. ebd.

42 Vgl. ebd.

43 Vgl. dazu a.a.O., S. 238.

44 Zu diesem Verweis von ihm, vgl. a.a.O., S. 241.

45 Vgl. bspw. Damasio, Antonio R.: Looking for Spinoza. Joy, sorrow and the feeling brain, London: Vintage 2004, S. 89. Vgl. ebenfalls Goldie, Peter: The emotions. A philosophical exploration, New York: Oxford University Press 2002, S. 4.

46 Dennett, Daniel C.: »Quining Qualia «, in: Philosophy of mind. Classical and contemporary readings, Chalmers, David J. [Hrsg.], New York: Oxford University Press [zuerst 1988]/2002, S. 240.

47 Ebd.

48 Ebd.

49 Churchland, Paul M.: »The Rediscovery of Light«, The Journal of Philosophy, [1996] Vol. 93, No. 5, S. 221, 226 und 227.

$50 \quad$ A.a.O., S. 221.
} 
anderen epistemischen Modalitäten zwar kritisiert, ${ }^{51}$ aber Qualia nur in Verbindung mit Introspektion bringt. So stellt er Qualia, implizit als angeblich blosse Fälle der Introspektion (»[...] the epistemic modalities that underwrite (what we loosely call) >introspection $[\ldots . . \ll)^{52}$ den Fällen exterozeptiver Sinneswahrnehmung gegenüber: »[...] the epistemic modalities of vision, taste, and audition ${ }^{53}$ Auf dieser Basis geht er dann von »inneren Qualia« aus, durch welche einige Reduktionsskeptiker hoffnungslos verführt worden seien: »And yet, philosophers have regularly been tempted here, some beyond redemption. [...] Nagel, Jackson, Searle, and Chalmers reject the proposed reduction of inner qualia to physical states of the brain «. ${ }^{4}$ Dementsprechend ist Churchlands Auswahl von Beispielen phänomenalen Erlebens insofern tendenziös, weil sie nur phänomenale Modalitäten der Introspektion ${ }^{55}$ und nicht einmal die einfachen und herkömmlichen Standardfälle phänomenaler Bewusstseinszustände der sinnlichen Exterozeption in Erwägung zieht:

Why should it be impossible a priori that the epistemic modality we call sintrospection < have some similar overlap with one or more of our other epistemic modalities? [...] One can tell by introspection that one's own bladder is full, but an ultrasound image will tell anyone the same thing. One can tell by introspection that and where one's retinal cells are photo-fatigued (we call it an safter image $<$ ), but that too is accessible by nonsubjective means. One can tell by introspection that the cochlear cells of one's inner ear are firing randomly (the condition is called stinnitus $<$ ), but others can access their behavior instrumentally. There are, of course, thousands more such examples. ${ }^{56}$

$5^{1}$ So kritisiert er: »None of our other epistemic modalities has any such distinction: they all access some aspect or other of the purely physical world. Why should introspection be any different? « Ebd.

52 A.a.O., S. 226.

53 Ebd. Der ganze Abschnitt, der aufzeigt, dass Churchland im Zusammenhang mit Qualia offenbar von einer Aufspaltung unseres epistemischen Zuganges zur Welt in >Introspektion< bzw. >inneren Qualia gegenüber anderen epistemischen Modalitäten auszugehen scheint, lautet folgendermassen: »Most importantly, there is no reason to expect that the current limits of the typical person's knowledge must mark the boundary of a distinct ontological domain. This is just as true, note, for the epistemic modalities that underwrite (what we loosely call) >introspection< as it is for the epistemic modalities of vision, taste, and audition. And yet, philosophers have regularly been tempted here, some beyond redemption. [...] Nagel, Jackson, Searle, and Chalmers reject the proposed reduction of inner qualia to physical states of the brain«. Ebd.

54 Ebd. [Hervorhebung E. E.].

55 Genauer handelt es sich bei Churchlands Beispielen des Empfindens einer vollen Blase bzw. eines Tinnitus Hörerlebens um Fälle der Interozeption bzw. um Wahrnehmungen von Reizen aus dem Körperinneren. Mehr zur Interozeption finden sich in Abschnitt 8.2.2.

$5^{6} \quad$ A.a.O., S. 227. 
Im Zusammenhang mit der Konzeption, dass Qualia intrinsisch sind, steht wohl auch die Idee von deren »Innerlichkeit «, ${ }^{57}$ mit der sie auch leicht in die >Schmuddelecke< des »Mysteriösen «,58 des »Obskurantismus,$^{59}$ des $»$ Ungewöhnlichen «, 60 eines bloss »idiosynkratischen Phänomens « (»idiosyncratic phenomenon « $)^{61}$ oder einfach als subjektive Innerlichkeit und Objekt unserer »introspizierende Alltagspsychologie ${ }^{62}$ abgedrängt werden können. Die Churchlands vergleichen in diesem Zusammenhang, die mit Qualia angeblich in Verbindung stehenden sintrospektiven Überzeugungen« sogar mit dem Sehen von dämonischen Geistern. ${ }^{63}$

Mit der damit verbundenen Optik einer angeblichen >Marginalitätく des Phänomenalen fällt es dann einfacher und scheint es legitim, die Aufmerksamkeit vom hard problem auf das easy problem zu lenken. Vielleicht geschieht das auch in der Hoffnung, dass das hard problem vielleicht nur aufgrund jener >mysteriösen Innerlichkeit< besteht. Doch die Konzeption,

57 Metzinger, Thomas: Subjekt und Selbstmodell. Die Perspektivität phänomenalen Bewusstseins vor dem Hintergrund einer naturalistischen Theorie mentaler Repräsentation, Paderborn: Mentis 1999, S. 37. Vgl. dazu auch Dennett: nach dem Qualia als letzte Bastion der »Innerlichkeit« des Mentalen (»Inwardness of our minds«) gedeutet werden. Dennett, Daniel C.: »Quining Qualia«, in: Philosophy of mind. Classical and contemporary readings, Chalmers, David J. [Hrsg.], New York: Oxford University Press [zuerst 1988]/2002, S. 229.

$5^{8} \quad$ Bzw. »mystery«, vgl. dazu Churchland, Paul M.: »The Rediscovery of Light«, The Journal of Philosophy, [1996] Vol. 93, No. 5, S. 226. Searle warnt diesbezüglich davor, dass wir uns nicht davon trügen lassen sollten, dass wir das »Problem der >Qualia«« einfach ignorieren können, indem wir uns vom Konstrukt leiten lassen, dass es ein informationsverarbeitendes Bewusstsein gebe, das für die Wissenschaft zugänglich sei und daneben noch ein mysteriöses phänomenales Bewusstsein (dem man wegen seiner angeblichen >Marginalität` getrost ausweisen kann). Vgl. dazu Searle, John R.: The mystery of consciousness, New York: New York Review Books 1997, S. 200.

59 Metzinger, Thomas: Subjekt und Selbstmodell. Die Perspektivität phänomenalen Bewusstseins vor dem Hintergrund einer naturalistischen Theorie mentaler Repräsentation, Paderborn: Mentis 1999, S. 239 .

6o So konstatiert Langsam, dass nach Peacock Qualia dafür benötigt werden, den subjektiven Charakter bestimmter ungewöhnlicher Erlebnisse auszuweisen. Vgl. Langsam, Harold L.: »Experiences, Thoughts, and Qualia«, Philosophical Studies, [200o] Vol. 99, No. 3, S. 269.

61 Churchland, Paul M.: »The Rediscovery of Light«, The Journal of Philosophy, [1996] Vol. 93, No. 5 , S. 223 .

62 Metzinger, Thomas: Subjekt und Selbstmodell. Die Perspektivität phänomenalen Bewusstseins vor dem Hintergrund einer naturalistischen Theorie mentaler Repräsentation, Paderborn: Mentis 1999, S. 37.

63 Vgl. Churchland, Paul M.: Matter and consciousness. A contemporary introduction to the philosophy of mind, Cambridge, Massachusetts: MIT Press 1988. Vgl. dazu auch Ramsey, William: »Eliminative Materialism«, in: The Stanford Encyclopedia of Philosophy, 2013, Online: http://plato.stanford.edu/archives/sum2o13/entries/materialism-eliminative/ [zuletzt geprüft am 25.09.2018], Abschnitt 3.1. 
dass Qualia nur »introspektive « ${ }^{64}$ Phänomene sind oder nur mit »Innerlichkeit« (»inwardness « $)^{65} \mathrm{zu}$ tun haben sollen, ist phänomenal inadäquat. Introspektivität ist keine notwendige Eigenschaft von phänomenalem Erleben, weil auch Sinneswahrnehmungen, die einen >extrospektiven bzw. exterozeptiven Bezug haben, Fälle von Qualia-Erleben sind: Nicht nur eine (introspektive) Imagination einer roten Wolke, sondern auch die (exterozeptive) Wahrnehmung einer weissen Wolke stellen Beispiele von phänomenalem Erleben dar:

$[\ldots]$ it is unnecessary to invoke $[\ldots]$ controversial claims as to the introspectible character of certain infrequently occurring perceptual experiences in order to argue for qualia, for it is certain obvious features of all perceptual experiences that provide us with reasons for believing in qualia. ${ }^{66}$

Die starren epistemische Bereiche von Intrinsität ${ }^{67}$ oder $»$ Innerlichkeit « 68 lassen sich, wenn wir dem Desiderat phänomenaler Adäquatheit treu bleiben wollen, nicht aufrechterhalten. Mit Landgrebe lässt sich die damit verbundene Alltagsintuition einer dualistischen Scheidung von >Introspektion< versus Wahrnehmung der Aussenwelt folgendermassen erklären: Wir sprechen im Hinblick auf die Momente eines Tagesverlaufes ganz selbstverständlich von $>$ Erlebnissen $<$. Wir finden uns durch das »Haben dieser Erlebnisse « ${ }^{69}$ bestimmt. Diese Erlebnisse scheinen abgrenzbare »Momente unseres Lebensverlaufes « ${ }^{70}$ zu sein. Deshalb glaubt man, es handle sich bei ihnen um »umgrenzbare $\mathrm{Zu}-$ stände unserer selbst « ${ }^{71}$ die uns bestimmen bzw. unsere Identität und unser >Innenleben< ausmachen würden: »Wir sind also geneigt, Erlebnis [sic!] als

64 Dennett, Daniel C.: »Quining Qualia«, in: Philosophy of mind. Classical and contemporary readings, Chalmers, David J. [Hrsg.], New York: Oxford University Press [zuerst 1988]/2002, S. 229 .

65 Ebd.

66 Langsam, Harold L.: »Experiences, Thoughts, and Qualia«, Philosophical Studies, [2000] Vol. 99, No. 3, S. 269 [Hervorhebung von E. E.]. Siehe auch Beaton, der bemerkt, dass Qualia nicht immer introspektiv sein müssen resp. dass es auch von der Konzeption von >Introspektion< abhängt, ob nicht-introspektive Qualia existieren. Vgl. Beaton, Michael: »Qualia and Introspection«, Journal of Consciousness Studies, [2009] Vol. 16, No. 5, S. 105.

67 Vgl. Dennett, Daniel C.: »Quining Qualia«, in: Philosophy of mind. Classical and contemporary readings, Chalmers, David J. [Hrsg.], New York: Oxford University Press [zuerst 1988]/2002, S. 229.

68 Ebd.

69 Landgrebe, Ludwig / [Hrsg.: Novotný, Karel]:Der Begriff des Erlebens. Ein Beitrag zur Kritik unseres Selbstverständnisses und zum Problem der seelischen Ganzheit, Würzburg: Königshausen \& Neumann [zuerst 1932]/2010, S. 2 O.

70 Ebd.

71 Ebd. 
etwas anzusehen, wodurch unser $>$ Inneres bestimmt ist $[\ldots] \ll .{ }^{72}$ Daraus entsteht eine Tendenz, qualitative Erlebnisse als etwas >Inneres< von einem $>$ Draussen $<$ zu scheiden, obschon das $>$ Draussen $<$ ja genau so durch sinnliches Erleben und darauf aufbauenden Interpretationen bzw. Urteilen erlebt wird. ${ }^{73}$ Landgrebe warnt vor dieser vorschnellen, intuitiven Spaltung von >Aussenwelt< versus >Innenwelt :

Wir dürfen nicht von vornherein gelten lassen, dass es sich [bei bewusstem Erleben] um die Sphäre der Immanenz in irgendeinem Sinne, um ein >Inneres dem gegenüber steht eine $>$ Aussenwelt $<$ [sic!], handle [...]. Vielmehr soll unter Bewusstsein und Sein bei Bewusstsein nichts verstanden werden als der rein deskriptiv erfassbare Tatbestand unseres Wachseins im Unterschied gegen das Schlafen und sonstige Modi der Bewusstlosigkeit [...]. ${ }^{74}$

Im Zusammenhang mit der Konzeption der >Privatheit von Gedanken und Empfindungen lässt sich mit Nagel dennoch feststellen, dass körperliche $\mathrm{Zu}$ stände oder objektive Dinge uns nicht immer bewusst sind, dagegen können bewusste psychische Zustände gar nicht anders als gewusst werden. ${ }^{75}$ Deshalb wird die so genannte »Autorität « ${ }^{76}$ der Ersten-Person-Perspektive bzw. der $»$ unmittelbare $\ll, 77 »$ privilegierte Zugang ${ }^{78}$ als weiteres zentrales Merkmal von Qualia aufgeführt. ${ }^{79}>$ Privilegiert heisst, nur ich kann erfahren, wie es

72 Ebd.

73 Vgl. dazu auch a.a.O., S. 5o.

74 Ebd. [Hervorhebung im Original und Ergänzung in Klammern von E.E.].

75 Vgl. dazu Nagel, Thomas: »Physikalismus «, in: Analytische Philosophie des Geistes, Bieri, Peter [Hrsg.], Weinheim: Beltz [zuerst 1965, engl.]/2007, S. 6o.

76 Vgl. McDonald, Cynthia: Introspection«, in: The Oxford Handbook of Philosophy of Mind, McLaughlin, Brian P. / Beckermann, Ansgar / Walter, Sven [Hrsg.], Oxford: Oxford University Press 2009, S. 741.

77 Z. B. Ebd.

78 So z.B. Robinson: »Mental states are characterised by two main properties, subjectivity, otherwise known as privileged access, and intentionality «. Robinson, Howard: »Dualism«, in: The Stanford Encyclopedia of Philosophy, 2013, Online: http://plato.stanford.edu/ archives/fall2013/entries/dualism/ [zuletzt geprüft am 25.09.2018], Abschnitt 5.1 [Hervorhebung von E. E.]. Vgl. dazu auch McDonald, Cynthia: »Introspection«, in: The Oxford Handbook of Philosophy of Mind, McLaughlin, Brian P. / Beckermann, Ansgar / Walter, Sven [Hrsg.], Oxford: Oxford University Press 2009, S. 741.

79 Vgl. dazu auch Pauen, der kritisiert, dass die Rede von einem »Zugang« in »hohem Masse metaphorisch« sei. Pauen, Michael: »Warum die Autorität der ersten Person das Erklärungslückenargument untergräbt«, in: Den eigenen Geist kennen. Selbstwissen, privilegierter Zugang und Autorität der ersten Person, Newen, Albert / Vosgerau, Gottfried [Hrsg.], Paderborn: Mentis 2005, S. 211. 
ist eine bestimmte Empfindung zu haben. ${ }^{80}$ Andere Personen können das Phänomenale meiner Empfindungen allenfalls nur erschliessen. ${ }^{81}$ Dieser $\mathrm{Zu}-$ gang, so wird kritisiert, sei aber nicht unvermittelt und auch nicht immun gegen Irrtümer. ${ }^{82}$ Die epistemische Bedeutung des privilegierten Zugangs wird vor allem in Verbindung mit der Konzeption der »Infallibilität «83 von Qualia in Frage gestellt. ${ }^{84}$ So bestreitet Pauen die Autorität der Ersten-PersonPerspektive, weil sich deren Irrtumsanfälligkeit nicht von unserem Zugang zu externen Tatsachen unterscheidet. ${ }^{85}$ Ebenfalls mit einer auf Urteile bezogenen Konzeption der »Infallibilität « ${ }^{86}$ von Qualia, versucht Dennett mit dem oben erwähnten Gedankenexperiment der hypothetischen Kaffeetester Chase und

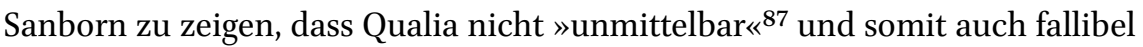
sind (und es deshalb keine Qualia geben soll). ${ }^{88}$

8o Dies mag wie ein Widerspruch gegenüber der oben kritisierten plakativen Unterscheidung einer reinen >Innerlichkeit< versus erlebten Eigenschaften der >Aussenwelt< anmuten. Die Konzeption der >Privilegiertheit bezieht sich jedoch sowohl auf Gedanken und Gefühlen und auch auf exterozeptiv, enterozeptive und auch introspektiv erlebte phänomenale Bewusstseinszustände: Ich weiss in einem privilegierten Sinne, wie es ist, wenn ich mir (introspektiv) einen roten Apfel vorstelle, nur ich weiss, wie es ist, wenn ich eine schwer zu definierende Körperempfindung habe (Enterozeption) und nur ich weiss mit Bestimmtheit, wie es ( für mich) ist, wenn ich meine Sinne nach >aussen $<$ richte (Exterozeption), und an einer Rose rieche oder den Geschmack von Ingwer schmecke, auch wenn sich von einer anderen Person erschliessen lässt, wie es für mich sein könnte.

81 Vgl. dazu a.a.O., S. 214.

82 Vgl. a.a.O., S. 211.

83 Metzinger, Thomas: Subjekt und Selbstmodell. Die Perspektivität phänomenalen Bewusstseins vor dem Hintergrund einer naturalistischen Theorie mentaler Repräsentation, Paderborn: Mentis 1999, S. 74. Vgl. ebenfalls McDonald, Cynthia:»Introspection«, in: The Oxford Handbook of Philosophy of Mind, McLaughlin, Brian P. / Beckermann, Ansgar / Walter, Sven [Hrsg.], Oxford: Oxford University Press 2009, S. 741.

84 So z.B. auch die von Metzinger erwähnte Gummihand-Illusion, die sich aufgrund einer Korrelation zwischen Inputs aus Gesichts- und Tastsinn erzeugen lässt und eine Fehlwahrnehmung im phänomenalen Selbstmodell darstellt. Vgl. dazu Metzinger, Thomas: Der Ego Tunnel. Eine neue Philosophie des Selbst: Von der Hirnforschung zur Bewusstseinsethik, Berlin: Berliner Taschenbuch Verlag [zuerst 20o9, engl.]/2011, S. 114.

85 Vgl. Pauen, Michael: »Warum die Autorität der ersten Person das Erklärungslückenargument untergräbt«, in: Den eigenen Geist kennen. Selbstwissen, privilegierter Zugang und Autorität der ersten Person, Newen, Albert / Vosgerau, Gottfried [Hrsg.], Paderborn: Mentis 2005, S. 212.

86 Dennett, Daniel C.: »Quining Qualia«, in: Philosophy of mind. Classical and contemporary readings, Chalmers, David J. [Hrsg.], New York: Oxford University Press [zuerst 1988]/2002, S. 233 .

87 Vgl. a.a.O., S. 244.

88 Vgl. a.a.O., S. 231-236. 
Genauer zeigen solche Beispiele über die Irrtumsanfälligkeit der ErstenPerson-Perspektive nur, dass unsere Urteile über phänomenale Zustände fallibel sind. ${ }^{89}$ An der Konzeption des privilegierten Zugangs zu unserem subjektiven Erleben ändert dies jedoch nichts. Denn der Begriff der >Infallibilität< muss nicht im Begriff des privilegierten Zugangs enthalten sein. ${ }^{90}$ Zur Erinnerung: >privilegiert < heisst lediglich, nur ich kann wissen, wie es ist, denjenigen phänomenalen Bewusstseinszustand zu erleben, den ich gerade erlebe. ${ }^{91}$ Die Konzeption des privilegierten Zugangs und die damit verbundene epistemische Asymmetrie zwischen der Ersten-Person- und der Dritte-PersonPerspektive hängt also eng mit dem epistemologischen problem of other minds zusammen. ${ }^{92}$ Das bedeutet, dass wir auch dann einen privilegierten Zugang zu einem phänomenalen Bewusstseinszustand haben, wenn wir über ihn ein falsches Urteil fällen, denn der qualitative Gehalt phänomenaler Bewusstseinszustände ist überzeugungsresistent. ${ }^{93}$ Auch mit meinem falschen Urteil über den phänomenalen Gehalt, den ich gerade erlebe, weiss nur ich, wie es ist, diesen (falsch beurteilten) Gehalt zu erleben. ${ }^{94}$ Dies trifft sogar dann zu, wenn wir (auf einer Urteilsebene) nicht von dem überzeugt sind, was wir erleben. Auch in diesem Fall können wir keine Revision des eigentlichen

89 Dennett zeigt in Quining Qualia in einem Beispiel mit den beiden Kaffeetestern nur auf, dass es auch aus der Erste-Person-Perspektive schwierig oder sogar unmöglich ist, eine $E r$ innerung an einen erlebten Geschmack exakt abzurufen. Daraus schliesst Dennett richtig, dass Urteile über (vergangene) Qualia fallibel sind. Vgl. a.a.O., S. 231. Ebenfalls ist es, wie Dennett feststellt, schwierig, bei der beschriebenen Veränderung des Geschmackerlebens von Chase und Sanborn zu bestimmen, ob sich nun das phänomenale Erleben des Geschmackes, ihre Urteile bzw. Reaktion über den Geschmack oder beides geändert hat. A.a.O., S. 232-233. Mehr zu dieser Kritik findet sich in den Abschnitten 9.2.1 und 9.2.3.

9o Vgl. dazu auch Schröder, Jürgen: »Qualia und Physikalismus«, Journal for General Philosophy of Science, [1997] Vol. 28, No. 1, S. 173.

91 Schröder beschreibt das so: »Alles, worum es hierbei geht, ist, dass das Wissen um ein Quale nichtinferentiell ist bei der Person, die das Quale hat und dass niemand anders (normalerweise) ein solches nichtinferentielles Wissen hat «. Ebd.

92 Hyslop beschreibt dies folgendermassen: »The epistemological problem is produced by the radical difference that holds between our access to our own experience and our access to the experience of all other human beings«. Hyslop, Alec: »Other Minds«, in: The Stanford Encyclopedia of Philosophy, 2013, Online: http://plato.stanford.edu/archives/ spr2013/entries/other-minds/ [zuletzt geprüft am 24.09.2018], Abschnitt 1.1.

93 Vgl. dazu Schildknecht, Christiane: »Phänomenales Erleben in Kunst und Literatur«, in: Kunst denken, Burri, Alex [Hrsg.], Paderborn: Mentis 2007, S. 92-93.

94 So besteht nach Schildknecht eines der drei strukturellen Merkmale phänomenaler Bewusstseinszustände in ihrem genannten »pour-soi-Charakter«. Qualia existieren nur je für mich bzw. je für dich. Sie sind untrennbar mit einer je eigenen subjektiven Perspektive und einem epistemischen Primat verknüpft. Vgl. ebd. 
Gehalts des Wahrgenommenen vollziehen. ${ }^{95}$ Das heisst, wir können den wahrgenommenen Gehalt selber, bspw. unsere Farbwahrnehmung beim Betrachten einer Benham-Scheibe, ${ }^{96}$ als etwas Farbiges erleben, auch wenn wir genau wissen, dass die Scheibe nicht farbig ist. Deshalb könnte man auch konstatieren, dass Qualia, im Hinblick auf den gerade erlebten (nicht erinnerten) phänomenalen Gehalt, >infallibel sind, denn auch sinnesphysiologische Fehlleistungen gehören zum phänomenalen Erleben. ${ }^{97}$ Urteile über erlebte oder erinnerte phänomenale Bewusstseinszustände sind jedoch fallibel. ${ }^{98}$

Qualia-Skeptiker/innen könnten hier unter Rückgriff auf das Privatsprachenargument Wittgensteins ${ }^{99}$ vielleicht noch einwenden, dass der private bzw. privilegierte Zugang zu phänomenalen Erleben trotzdem nicht vollständig gegeben sein könne, wenn doch mindestes unsere Urteile über sie fallibel sind.

95 Vgl. dazu ebd.

96 Zur Erinnerung: eine Benham-Scheibe ist eine schwarz-weisse Scheibe, die in Rotation gebracht, durch eine optische Täuschung als etwas >Farbiges< wahrgenommen wird. Vgl. dazu Staudacher, Alexander: Phänomenales Bewusstsein als Problem für den Materialismus, Berlin: De Gruyter 2002, S. 324.

97 Aus diesem Grund kann man mit Schildknecht feststellen, dass phänomenales Erleben ein nicht-propositionales Wissen über mentale Zustände ist, nämlich ein Wissen wie es ist. Diese Wissensform weist eine pour-soi-Struktur auf, d.h. sie ist insofern infallibel, dass das Wissen wie es ist nicht von der Richtigkeit der Urteile bzw. Propositionen, die über das Erleben gemacht werden, abhängt. Vgl. dazu auch Schildknecht, Christiane: Sense and self. Perspectives on nonpropositionality, Paderborn: Mentis 2002, S. 224-226. Phänomenale Bewusstseinszustände sind also, auch wenn sie epistemisch gehaltvoll sind, und somit eine Wissensform darstellen, nicht auf Wahrheit in einem propositionalen Sinne bezogen. Für ein phänomenales Wissen, wie es ist, bspw. eine Benham-Scheibe fälschlicherweise $>$ farbig zu sehen $»[\ldots]$ ist propositionales Wissen weder notwendig noch hinreichend [...]«. Schildknecht, Christiane: Aspekte des Nichtpropositionalen, Bonn: Bouvier 1999, S. 11. Vgl. auch a.a.O., S. 8-10.

98 Analog dazu kann unsere »Irrtumsanfälligkeit« gegenüber phänomenalem Erleben auch nicht mit unserer Irrtumsanfälligkeit im Hinblick auf Urteile über erlebte Gegenstände verglichen werden: One could have something that felt like water without it being water; but one could not have something that felt like pain without it being pain. Pain's feel is essential to it«. Chalmers, David J.: The conscious mind. In search of a fundamental theory, New York: Oxford University Press 1996, S. 147 [Hervorhebung im Original]. Vgl. dazu auch Schildknecht, Christiane: Sense and self. Perspectives on nonpropositionality, Paderborn: Mentis 2002, S. 209.

99 Weil wir »kein Kriterium für die Richtigkeit« einer hinweisende Definition auf ein inneres Erlebnis hätten. Wittgenstein, Ludwig / [Hrsg.: Schulte, Joachim]: Philosophische Untersuchungen, Frankfurt am Main: Suhrkamp [zuerst 1953]/2003, S. 877 [§258]. Vgl. auch Gebauer, Gunter / Stuhldreher, Anna: Wittgenstein: Das Sprachspiel der Emotionen«, in: Klassische Emotionstheorien. Von Platon bis Wittgenstein, Landweer, Hilge / Renz, Ursula [Hrsg.], Berlin: De Gruyter 2012, S. 633 . 
Denn das würde ja bedeuten, dass subjektives Erleben (z.B. im Falle erinnerter Qualia) unserer eigenen Kognition nicht unmittelbar zugänglich ist. ${ }^{100}$ Damit wären doch Qualia als etwas >Privates $<$ hinfällig?

Doch selbst wenn wir dieser Argumentation folgten (obschon sie den Unterschied zwischen Erleben und Urteilen über Erleben auslässt und sie sich nur auf Fälle erinnerter Qualia anwenden lässt) können wir festhalten, dass auch der Begriff der >Privatheit keine notwendige Bedingung für phänomenales Erleben ist. Um dies zu begründen, machen wir folgendes Gedankenexperiment: Wenn es einem Hirnforscher gelänge, bspw. durch ein Interface, der mit Ihrem visuellen Cortex verbunden wäre, die Farben, Blumen und Strände, die Sie sich vorstellen, auf einem Bildschirm, der auf Ihrem Kopf angebracht ist, sichtbar zu machen, so wären diese Vorstellungen nicht mehr ausschliesslich in Ihrem eigenen privaten, geschützten Raum des Subjektiven. Analog zu einem Comic, bei dem auch das >private〈Erleben einer Figur durch Denkblasen für die Leser/ innen sichtbar ist, würde im Gedankenexperiment ein Teil des Innenlebens von Ihnen als Versuchsperson zu intersubjektiv erlebbaren (Vorstellungs-) Gehalten. Wäre das Gedankenexperiment technisch realisierbar, so hätte dies das Ende der prinzipiellen >Privatheit unseres Innenlebens zur Folge. ${ }^{101} \mathrm{Im} \mathrm{Zu}$ sammenhang mit dem problem of other minds kann man hier natürlich noch einwenden, dass wir auf den Bildschirmen, welche das Innenleben von Ihnen (und anderen Versuchspersonen) zeigen würden, zwar sehen, was Sie sich vorstellen, aber nicht wie Sie jene Vorstellung genau erleben. Um also eine weitere Stufe von Privatheit zu durchbrechen, können wir uns vorstellen, dass das Interface welches auf den visuellen Cortex von Ihnen zugreift, statt mit einem Bildschirm, direkt mit dem visuellen Cortex der Versuchsleiterin verbunden wäre. Die Versuchsleiterin hätte dann, cum crano salis, (phänomenale) Einblicke in das >private< visuelle Vorstellen bzw. Erleben von Ihnen - auf jeden Fall wird der Aspekt der >Privatheit< bzw. der >Privilegiertheit< durch dieses Gedankenexperiment stark relativiert. Mindestens Sie und andere Versuchspersonen wären der Privatheit ihres >Innenlebens« zu einem beträchtlichen Mass beraubt. Aber hiesse das, dass Sie und diese Menschen dann gar nichts

100 Vgl. dazu z.B. Dennett, Daniel C.: »Quining Qualia«, in: Philosophy of mind. Classical and contemporary readings, Chalmers, David J. [Hrsg.], New York: Oxford University Press [zuerst 1988]/2002, S. 244.

101 Auch wenn dies bei komplexen Gedanken und Gefühlen vielleicht unmöglich ist, so könnte dies mindestens im Bereich einfacher visueller oder anderer introspektiver Vorstellungsinhalte prinzipiell möglich sein. Mit dem Fortschreiten der kognitiven Neurowissenschaften und der Neuropsychologie wäre es vielleicht auch denkbar, dass auch komplexe Gehalte von Versuchspersonen angezeigt werden könnten. Für das Gedankenexperiment ist die konkrete technische Realisierbarkeit ohnehin zweitrangig. 
mehr erleben würden? Würde sich, wegen der durch diesen technischen Eingriff zerstörten >Privatheitく des subjektiven Erlebens, phänomenales Erleben nur weil andere Zugriff darauf haben - wie durch einen Zauber auflösen? Nein, selbst wenn die Versuchsleiter alle visuellen Vorstellungen oder sogar alle Gefühle und Gedanken aller Versuchspersonen sehen könnten, so könnte man doch nicht behaupten, dass letztere deswegen zu einem Zombie mutieren würden. Der phänomenale Charakter ihrer Visualisierungen von Traumstränden und die Erlebnisqualität der dabei ausgelösten Emotionen, blieben nichtsdestotrotz erhalten. Das bedeutet aber, dass phänomenale Bewusstseinszustände auch nicht auf den angeblich zwingenden Qualia-Eigenschaften der $>$ Privatheit< oder der >Privilegiertheit< gründen müssen .

Weil phänomenales Erleben nicht mit unseren Urteilen über sie gleichzusetzen ist, werden Qualia oft auch als »unbeschreiblich« (bzw. »ineffable «),102 $»$ nicht-begrifflich $\ll, 103 »$ non-conceptual $\ll^{104} \gg$ oder nicht-epistemisch $\aleph^{105}$ charakterisiert. Wird Nicht-Begrifflichkeit jedoch als notwendige Qualia-Eigenschaft vorausgesetzt, so wird der Qualia-Begriff insofern eingeengt, als damit impliziert wird, unserer Denkvorgänge, hätten aufgrund ihres Bezuges zu

102 So sieht bspw. Robinson in der Unbeschreiblichkeit (»ineffability«) eine oft erwähnte Eigenschaft von Qualia: »A cannot convey to B what kind of qualia she has. That is, there is absolutely nothing she could do to satisfy B [...]. This property of qualia is often referred to as their ineffability«. Robinson, William S.: »Qualia realism «, in: A Field Guide to the Philosophy of Mind, SIFA, Società Italiana Filosofia Analitica, 2002, Online: http:// host.uniroma3.it/progetti/kant/field/qr.htm [zuletzt geprüft am 25.09.2018], Abschnitt »Three Further Properties«. Vgl. dazu auch Lycan, William: »Representational Theories of Consciousness«, in: The Stanford Encyclopedia of Philosophy, 2008, Online: http://plato. stanford.edu/archives/fall2oo8/entries/consciousness-representational/ [zuletzt geprüft am 28.09.2018], Abschnitt 5.o.

103 Z.B. Willaschek, Marcus: „Wahrnehmung VII (Analytische Philosophie)«, in: Historisches Wörterbuch der Philosophie. Band 12: W-Z, Ritter, Joachim / Kranz, Margarita [Hrsg.], Darmstadt: Wissenschaftliche Buchgesellschaft 1971-2007, S. 231.

104 So z.B. beim unter dem Kürzel PANIC bekannt geworden Qualia-Begriff von Tye: »[...] the phenomenal character of an experience is one and the same as its poised abstract non-conceptual intentional (or representational) content or its PANIC, for short [...]«. Tye, Michael: »Representationalist Theories of Consciousness «, in: The Oxford Handbook of Philosophy of Mind, McLaughlin, Brian P. / Beckermann, Ansgar / Walter, Sven [Hrsg.], Oxford: Oxford University Press 2009, S. 259. Vgl. ebenfalls Pitt, David: »Mental Representation«, in: The Stanford Encyclopedia of Philosophy, 2008, Online: http://plato. stanford.edu/archives/fall20o8/entries/mental-representation [zuletzt geprüft am 25.09.2018], Abschnitt 3.o.

105 Z.B. Lanz, Peter: Das phänomenale Bewusstsein. Eine Verteidigung, Frankfurt am Main: Klostermann 1996, S. 6o. 
Begriffen, keinerlei phänomenale Eigenschaften. ${ }^{106}$ Im nächsten Abschnitt werde ich ausweisen, dass sich diese Behauptung bei einem phänomenal adäquaten Qualia-Begriff ebenfalls nicht halten lässt. ${ }^{107}$

Wenn es bei Qualia um phänomenales Erleben gehen soll, und nicht nur um hypothetische isolierte Eigenschaften des Erlebens, dann sind solche Vereinfachungen besonders dann nicht zulässig, wenn auf einer phänomenal derart inadäquaten Grundlage Qualia geleugnet werden. Im Lichte der Problemoptik der phänomenalen Achse und dem damit verbundenen Desiderat phänomenaler Adäquatheit lässt sich also monieren, dass die bisherige Diskussion an einem viel zu eng gefassten und deswegen auch phänomenal inadäquaten Begriff qualitativen Erlebens leidet. ${ }^{108}$ Aus diesem Grund wird der

106 So stellt Pitt fest:»[...] thoughts, which are composed of concepts [...] have no phenomenal (`what-it's-like $<$ ) features (`qualia $<$ ) [...] «. Pitt, David: »Mental Representation«, in: The Stanford Encyclopedia of Philosophy, 2008, Online: http://plato.stanford.edu/archives/ fall2008/entries/mental-representation [zuletzt geprüft am 25.09.2018], Abschnitt 3.o.

107 An dieser Stelle sei nur schon mal darauf verwiesen, dass es doch auch irgendwie ist, ganz bestimmte Gedanken, oder überhaupt Denkvorgänge, zu haben. Diesbezüglich konstatiert Chalmers: »I am talking about the subjective quality of experience: what it is like to be a cognitive agent«. Chalmers, David J.: The conscious mind. In search of a fundamental theory, New York: Oxford University Press 1996, S. 6.

108 Weitere Beispiele zum klassischen, zumeist monosensualen Verständnis von Qualia finden sich bei Lewis, Clarence I.: Mind and the World-Order. Outline of a Theory of Knowledge, New York: Scribner 1929, S. 6o, 121 und 124. Oder: Levine, Joseph: »Qualia: Intrinsisch, relational - oder was? «, in: Bewusstsein. Beiträge aus der Gegenwartsphilosophie, Metzinger, Thomas [Hrsg.], Paderborn \& Zürich: Schöningh [zuerst 1995, engl.]/1995, S. 329. Oder: Slaby, Jan: Gefühl und Weltbezug. Die menschliche Affektivität im Kontext einer neo-existentialistischen Konzeption von Personalität, Paderborn: Mentis 2008, S. 316. Oder: Lycan, William: »Representational Theories of Consciousness«, in: The Stanford Encyclopedia of Philosophy, 2008, Online: http://plato.stanford.edu/archives/ fall2008/entries/consciousness-representational/ [zuletzt geprüft am 28.09.2018], Abschnitt 2.3. Oder: Metzinger, Thomas [Hrsg.]: Grundkurs Philosophie des Geistes. Band 1: Phänomenales Bewusstsein, Paderborn: Mentis 2007, S. 203. Oder: Metzinger, Thomas: Subjekt und Selbstmodell. Die Perspektivität phänomenalen Bewusstseins vor dem Hintergrund einer naturalistischen Theorie mentaler Repräsentation, Paderborn: Mentis 1999, S. 88. Oder: Shoemaker, Sydney: »The inverted spectrum«, in: The Journal of Philosophy, [1982] Vol. 79, No. 7, S. 367. Oder: Lanz, Peter: Das phänomenale Bewusstsein. Eine Verteidigung, Frankfurt am Main: Klostermann 1996, S. 98. Oder: Heckmann, HeinzDieter: »Qualia-basierte Argumente gegen den Materialismus«, in: Bewusstsein und Repräsentation, Esken, Frank / Heckmann, Heinz-Dieter [Hrsg.], Paderborn: Mentis 1999, S. 33o. Oder: Lorenz, Kuno: »Qualia«, in: Enzyklopädie Philosophie und Wissenschaftstheorie. Band 3: P-So, Mittelstrass, Jürgen [Hrsg.], Stuttgart: Metzler 2004, S. 426-427. Oder: Raffman, Diana: »Über die Beharrlichkeit der Phänomenologie«, in: Bewusstsein. Beiträge aus der Gegenwartsphilosophie, Metzinger, Thomas [Hrsg.], Paderborn \& Zürich: Schöningh 1995, S. 354. Oder: Searle, John R. / [Übers.: Gavagai, Harvey]: Die Wiederentdeckung des Geistes, München: Artemis-Verlag \& Winkler [zuerst 1992, engl.]/1993, S. 59 
herkömmliche Qualia-Begriff auch nur einem Bruchteil aller phänomenalen Tatsachen gerecht. So konstatiert Strawson, dass die Diskussion dazu neigt, sich auf einen extrem engen Begriff von Erleben zu beziehen:»[...] in certain contexts of discussion, we may still be inclined to appeal to an excessively restricted notion of what experience is «.109 Nach Slaby liegt der Grund für die künstliche Einengung des Phänomenalen auf vornehmlich monosensuale Schemata von Erleben in der Absicht, »[...] die sisolierte< Thematisierung des qualitativen Charakters von Erfahrung zu erleichtern «.10 Zudem hat die etablierte Qualia-Debatte zu einer Verengung des Fragehorizonts geführt und »[...] teilweise hochgradig selbstreferentielle Themenkreise erzeugt, in denen immer feingliedrigere Binnendifferenzierung vorgenommen werden, deren Relevanz für übergreifende Fragestellungen $[. .$.$] «111 oft nicht mehr erkennbar$ sind. Damit verbunden besteht die Tendenz, die »empirische Breite qualitativer Phänomene «112 einzuengen auf wenige immer wiederkehrende Beispiele, wie z.B. die Farbwahrnehmung im Gedankenexperiment des epistemischen Argumentes um »Mary«. ${ }^{13}$ Wenn die theoretische Analyse, wie es häufig der Fall

und 277. Oder: Staudacher, Alexander: Phänomenales Bewusstsein als Problem für den Materialismus, Berlin: De Gruyter 2002, S. 3 und 62-65. Oder: Walter, Sven: »Allgemeine Einleitung: Phänomenales Bewusstsein: Unlösbares Mysterium oder seriöses wissenschaftliches Problem?«, in: Qualia. Ausgewählte Beiträge, Heckmann, Heinz-Dieter / Walter, Sven [Hrsg.], Paderborn: Mentis 2001, S. 13 .

109 Strawson, Galen: Mental reality, Cambridge, Massachusetts: MIT Press 1994, S. 8. Vgl. dazu auch Soldati, der feststellt, dass phänomenales Bewusstsein gewöhnlich nur auf einen ziemlich begrenzten Bereich psychischer Phänomene angewandt wird und dass selbst Positionen des phänomenalen Realismus, kognitiven mentalen Zuständen, also z.B. Überzeugungen und Urteile, keinen spezifischen phänomenalen Charakter zuschreiben würden. Vgl. Soldati, Gianfranco: »Begriffliche Qualia. Zur Phänomenologie der Bedeutung «, in: Anatomie der Subjektivität. Bewusstsein, Selbstbewusstsein und Selbstgefühl, Grundmann, Thomas / Hofmann, Frank / Misselhorn, Catrin et al. [Hrsg.], Frankfurt am Main: Suhrkamp 2005, S. 144. Darauf, dass in der Diskussion zum Qualia-Begriff Wahrnehmungen und Empfindungen im Vordergrund stehen, verweist auch Staudacher, weshalb er die Frage, ob Gedanken, »spezifische `Gedankenqualia«« haben, offen lässt. Vgl. Staudacher, Alexander: Phänomenales Bewusstsein als Problem für den Materialismus, Berlin: De Gruyter 2002, S. 5 .

110 Slaby, Jan: Gefühl und Weltbezug. Die menschliche Affektivität im Kontext einer neoexistentialistischen Konzeption von Personalität, Paderborn: Mentis 2008, S. 316.

111 Jung, Matthias / Heilinger, Jan-Christoph [Hrsg.]: Funktionen des Erlebens. Neue Perspektiven des qualitativen Bewusstseins. Band 5: Humanprojekt, Berlin: De Gruyter 2009, S. 7 .

112 Ebd.

113 Jackson, Frank: »What Mary Didn't Know«, The Journal of Philosophy, [1986] Vol. 83, No. 5, S. 291-295. 
ist (z.B. bei Tye), ${ }^{114}$ bestenfalls kurz die Breite phänomenaler Erfahrung erwähnt, sich dann aber rasch auf wenige Beispieltypen, wie Schmerz und Farbwahrnehmung konzentriert, dann leidet die Debatte an einer phänomenal inadäquaten Konzeption, was das Explanandum »Qualia« ist - oder wie Heilinger und Jung es nennen: es stellt sich ein »bias«115 ein, worin das Phänomenale besteht. ${ }^{116}$ Mit einem alles Subjektive fürchtenden Scheuklappenblick solcher Bedeutungseinengungen wird phänomenales Bewusstsein nicht mehr in adäquater Weise als Explanandum des hard problem erkannt. Infolgedessen wird Phänomenales in verschiedenen Gradierungen vernachlässigt, bezweifelt ${ }^{117}$ oder sogar, wie bei Dennett, explizit ausgeklammert. Deshalb überrascht es nicht, dass Philosophen, die behaupten, Qualia würden nicht >existieren<, nicht selten einen besonders engen Qualia-Begriff verwenden. ${ }^{118}$ Genau aus diesem Grund sollten auch nach Tye eliminativistische QualiaAnalysen vorsichtig interpretiert werden. ${ }^{119}$

Es stellt sich nämlich die Frage, was mit solchen phänomenal inadäquaten Begriffskreationen eigentlich genau verneint wird. Beispielsweise versucht

114 Vgl. Tye, Michael: »Qualia«, in: The Stanford Encyclopedia of Philosophy, 2007, Online: http://plato.stanford.edu/archives/fall2007/entries/qualia/ [zuletzt geprüft am 25.09.2018], Abschnitt 2.0-7.0.

115 Jung, Matthias / Heilinger, Jan-Christoph [Hrsg.]: Funktionen des Erlebens. Neue Perspektiven des qualitativen Bewusstseins. Band 5: Humanprojekt, Berlin: De Gruyter 2009, S. 8.

116 Auch Van Gulick verweist darauf, dass Qualia oft mit basalen Empfindungen (»raw feels«) bzw. Geschmacks- oder Farbwahrnehmung gleichgesetzt werden. Van Gulick kritisiert diese enge Auffassung von Qualia: »The relevant sort of qualitative character is not restricted to sensory states, but is typically taken to be present as an aspect of experiential states in general [...]《. Van Gulick, Robert: »Consciousness«, in: The Stanford Encyclopedia of Philosophy, 2014, Online: http://plato.stanford.edu/archives/spr2o14/ entries/consciousness/ [zuletzt geprüft am 17.09.2018], Abschnitt 4.2.

117 Vgl. z.B. Tim Crane, der auf einen »unverfänglichen« (»innocuous«) Begriff von Qualia verweist, nach welchem ein mentaler Zustand bereits Qualia aufweist, wenn er ganz einfach bewusst ist. Bei einer solchen Verwendungsweise des Begriffes habe Ned Block recht, wenn er sage, dass die Existenz von Qualia gar nicht bestritten werden könne. Demgegenüber steht der engere, »demanding sense« von Qualia, wie von Dennett und Jackson bei welchem es nicht mehr sicher sei, dass Qualia existieren. Vgl. Crane, Tim: »The origins of qualia«, 20oo, Online: http://sas-space.sas.ac.uk/220/1/The\%20origins\%20of\%2oqualia.pdf [zuletzt geprüft am 24.09.2018], S. 21.

118 Vgl. dazu Tye, Michael: »Qualia«, in: The Stanford Encyclopedia of Philosophy, 2007, Online: http://plato.stanford.edu/archives/fall2007/entries/qualia/ [zuletzt geprüft am 25.09.2018], Abschnitt 1.o.

119 Vgl. ebd. 
Dennett mit Hilfe von »Intuitionspumpen« (»intuition pumps«) $)^{120}$ nachzuweisen, dass es - nach dem vom ihm eng definierten Sinne - kein phänomenales Erleben >gibt<, sondern nur »relationale, extrinsische Eigenschaften ${ }^{121}$ In diesem Zusammenhang kritisiert aber Johnsen, dass Dennett in seinem Aufsatz Quining Qualia ${ }^{122}$ die Unterscheidung zwischen zwei Zielen, die er verfolgt, nicht durchzieht: a.) zu zeigen, dass Qualia nicht >existieren<; b.) auszuweisen, dass das cartesianische Theater-Modell des Bewusstseins falsch sei. So bezieht sich Dennetts enge Qualia-Konzeption nur auf das cartesianische Theater-Modell des Bewusstseins. ${ }^{123}$ Folglich lässt sich mit Johnsen die im Zusammenhang mit dem Desiderat phänomenaler Adäquatheit zentrale Frage stellen, warum »Dennett's four properties «124 überhaupt beibehalten werden sollen. ${ }^{125}$ Johnsen kritisiert weiter, dass Dennetts Charakterisierung von Qualia wenig innovativ sei, ja eher eine Beschreibung sei, die dem » common sense« «26 entspringe. Selbst wenn Dennett seinen eigenen vierfältigen Qualia-Begriff und das cartesianische Theater-Modell des Bewusstseins widerlegt hätte, so steht sein darauf aufbauender induktiver Schluss, dass es deshalb keine, wie auch immer konzipierte Qualia bzw. kein phänomenales Erleben >gibt<, auf sehr wackeligen Beinen: Warum soll die (geschenkte) Widerlegung eines essentialistischen, engen, wenig innovativen und erst noch phänomenal inadäquaten »vierfältigen «127 Qualia-Begriffs, zur Folge haben, dass damit phänomenales Erleben bzw. Qualia in toto >widerlegt` sind?

Dennetts Behauptung, dass die Widerlegung seines eignen »vierfältigen $«^{128}$ Qualia-Begriffs (bzw. des cartesianischen Theater-Modell des Bewusstseins) den Nachweis dafür erbringe, dass es keine Qualia `gibt‘, kann sogar mit dem seltsamen Schluss verglichen werden, dass die Widerlegung eines élan vital belege, dass es keine lebenden Organismen gibt: »But refuting the latter [das cartesianische Theater-Modell des Bewusstseins] could no more establish that

120 Dennett, Daniel C.: »Quining Qualia «, in: Philosophy of mind. Classical and contemporary readings, Chalmers, David J. [Hrsg.], New York: Oxford University Press [zuerst 1988]/2002, S. 229 .

121 Ebd.

122 Vgl. a.a.O., S. 226-246.

123 Vgl. Johnsen, Bredo: »Dennett on Qualia and Consciousness: A Critique«, Canadian Journal of Philosophy, [1997] Vol. 27, No. 1, S. 54.

124 Ebd. Johnsen bezieht sich hier auf die vier Eigenschaften des Qualia-Begriffs von Dennett.

125 Vgl. ebd.

126 Ebd.

127 Dennett nennt diesen $»[\ldots]$ my suggested fourfold essence of qualia [...] «. Dennett, Daniel C.: »Quining Qualia«, in: Philosophy of mind. Classical and contemporary readings, Chalmers, David J. [Hrsg.], New York: Oxford University Press [zuerst 1988]/2002, S. 229.

128 Ebd. 
there are no qualia than the refutation of elan vital theory established that there are no living things «. ${ }^{129}$ Dennett geht zwar sehr kurz auf dieses Problem ein, indem er sich überlegt, dass seine >Widerlegung $<$ von Qualia, auf der Basis jenes engen Qualia-Begriffs mit der >Unterwerfung eines Strohmannes< bzw. mit einem Strawman-Argument verglichen werden könnte: »They think I am setting up and knocking down a strawman, and ask in effect: > who said qualia are ineffable, intrinsic, private, directly apprehensible ways things seem to one? «.130 Dennett weiss also, dass sein Versuch, Qualia zu widerlegen, auch als sStrohmann-Argument< gesehen werden können. Ein >Strohmann-Argument< zeichnet sich bekanntlich dadurch aus, dass mit ihm, anstatt auf die Argumente des Gegners gebührend einzugehen, gegen einen fiktiven Gegner argumentiert wird. Im Gegensatz zu einer realistischen und differenzierten Darstellung der gegnerischen Position, wird bspw. dem Gegner ein ganz bestimmter, phänomenal inadäquater und enger Qualia-Begriff unterstellt. Der fiktive Gegner wird metaphorisch deshalb als `Strohmann< bezeichnet, weil ein solcher (da es ihn, als realen Gegner, gar nicht gibt), sich nicht zur Wehr setzen kann und es deshalb besonders leicht ist, ihn zu >widerlegen<. So gesteht Dennett zwar kurz ein, dass sein »vierfältiger «131 Qualia-Begriff als tendenziös aufgefasst werden könnte: »[...] my suggested fourfold essence of qualia may strike many readers as tendentious [...] «. ${ }^{132}$ Aus diesem Grund geht er schnell auf den, seiner Meinung nach $»$ milderen $\aleph^{133}$ Qualia-Begriff von Shoemaker ein. Bezeichnenderweise wird Dennett mit seiner Eliminationsstrategie schon bei diesem, nur leicht erweiterten und immer noch auf Sinneserlebnissen beschränkten Qualia-Begriff ${ }^{134}$ um einiges vorsichtiger: »Surely I do not mean to

129 Johnsen, Bredo: »Dennett on Qualia and Consciousness: A Critique«, Canadian Journal of Philosophy, [1997] Vol. 27, No. 1, S. 54 [Ergänzung in Klammern von E.E.].

130 Dennett, Daniel C.: »Quining Qualia «, in: Philosophy of mind. Classical and contemporary readings, Chalmers, David J. [Hrsg.], New York: Oxford University Press [zuerst 1988]/2002, S. 229 .

131 Ebd.

132 Ebd.

133 Ebd.

134 Dennett zitiert jenen »scheinbar milderen«, alternativen Qualia-Begriff von Shoemaker folgendermassen: »It may be instructive to consider, briefly, an apparently milder alternative: qualia are simply sthe qualitative or phenomenal features of sense experience[s], in virtue of having which they resemble and differ from each other, qualitatively, in the ways they do [...] «. Ebd. Vgl. dazu Shoemaker, Sydney: »The inverted spectrum«, The Journal of Philosophy, [1982] Vol. 79, No. 7, S. 367. [Zitiert nach Dennett Dennett, Daniel C.: »Quining Qualia«, in: Philosophy of mind. Classical and contemporary readings, Chalmers, David J. [Hrsg.], New York: Oxford University Press [zuerst 1988]/2002, S. 229.] 
deny those features! [...] it all depends on what >qualitative or phenomenal comes to ${ }^{135}$

Was nun aber besonders bemerkenswert ist, ist der Umstand, wie marginal Dennett auf das Desiderat phänomenaler Adäquatheit bzw. die Frage, wie Phänomenalität definiert wird, eingeht. Er widmet dieser Frage, die für seine Argumentation zu einem schwerwiegenden Problem werden könnte, bzw. wird, gerade nur knapp eine halbe Seite. ${ }^{136}$ Unter dem »scheinbar milderen «137 Qualia-Begriff von Shoemaker kann sich Dennett nämlich nichts vorstellen: Shoemakers Unterscheidung zwischen qualitativer Differenz/Ähnlichkeit und intentionaler Differenz/Ähnlichkeit ist für Dennett nur im Hinblick auf Intentionalität verständlich, da es sich bei Letzterem um die Differenzen bzw. Ähnlichkeiten von Eigenschaften handle, die ein Erlebnis repräsentieren. ${ }^{138}$ Shoemakers Begriff des Qualitativen bzw. Phänomenalen ist für Dennett offenbar ein Rätsel: »[...] but what then of sphänomenal<? Among the nonintentional (and hence qualitative?) properties of my visual states are their physiological properties. Might these very properties be the qualia Shoemaker speaks of? «.139 In der Folge kommt Dennett aber selbstverständlich auf die Idee, dass Shoemaker mit der Begrifflichkeit >phänomenaler Eigenschaften< wohl nicht auf physiologische Zustände verweisen will. Das einzige, was sich aber Dennett schlussendlich unter »nicht-intentionalen«bzw. phänomenalen Eigenschaften vorstellen kann, ist, dass sie ein Verhalten beeinflussen ${ }^{140}$ können und dass sie der »Introspektion zugänglich « (»accessible to introspection«) ${ }^{141}$ seien. Diese Charakterisierung phänomenaler Eigenschaften sind für ihn jedoch nur ein »vortheoretisches Konstrukt « (»pretheoretical construal «). ${ }^{142}$ Nun zeichnet sich definitiv aus, dass Dennett sich gar nichts - ausser seinem eigenen vierfältigen Begriff - unter Qualia bzw. phänomenalem Erleben vorstellen kann:

\footnotetext{
135 Ebd. [Hervorhebung im Original].

136 Vgl. ebd.

137 Ebd.

138 Vgl. ebd.

139 Ebd.

140 So konstatiert er: »[...] another non-intentional similarity [neben ihren physiologischen Eigenschaften] some of my visual states share is that they tend to make me think about going to bed «. Ebd.

141 Ebd.

142 Ebd.
} 
The term phenomenal means nothing obvious and untendentious to me and looks suspiciously like a gesture in the direction leading back to ineffable, private, directly apprehensible ways things seem to one. ${ }^{143}$

Auf dieser Grundlage, eines nicht weiter ausgearbeiteten Qualia-Begriffs und einer weitgehenden Ignorierung der Frage, was unter Phänomenalität verstanden werden soll, geht er im weiteren Verlauf seines ausführlichen Argumentationsstranges mit fünfzehn »Intuitionspumpen« natürlich ausschliesslich von seinem inadäquaten und engen Qualia-Begriff aus. So ist es auch nicht besonders erstaunlich, dass er zu folgendem Fazit gelangt:

So when we look one last time at our original characterization of qualia, as ineffable, intrinsic, private, directly apprehensible properties of experience, we find that there is nothing to fill the bill. ${ }^{144}$

\subsection{Skizzierung eines phänomenal adäquaten Qualia-Begriffs}

Der in der bisherigen Kontroverse um das hard problem verwendete QualiaBegriff wird dem Desiderat phänomenal Adäquatheit nicht gerecht. Zumeist übersieht man allein schon innerhalb der für Qualia noch üblichen und akzeptierten Kategorie sensorischer Wahrnehmungen der Außenwelt (Exterozeption) wichtige Bereiche phänomenalen Erlebens (vgl. Abschnitt 8.2.1). Auf diese Lücke in der Qualia-Diskussion soll jedoch nur kurz eingegangen werden, da die mit ihr verbundene phänomenale Inadäquatheit eher >quantitativer Natur ist und ein weniger schwerwiegendes Problem darstellt, als ein Ignorieren von fundamentalen Bereichen menschlichen Erlebens.

In diesem Kapitel soll nämlich gezeigt werden, dass bestimmte zentrale und wichtige Kategorien menschlichen Erlebens in der herkömmlichen QualiaDiskussion fast vollständig oder sogar gänzlich ausser Acht gelassen werden. Denn neben den herkömmlichen Beispielen von Sinneswahrnehmung und Schmerzempfindungen lassen sich weitere fundamentale Formen des Erlebens, wie emotionale, gedankliche und intentionale Zustände ausweisen. So muss ein phänomenal adäquater Qualia-Begriff auch das bewusste Erleben von Emotionen, die emotiven Modi des Denkens sowie phänomenale Aspekte des Denkens und des Intentionalen berücksichtigen. In Anbetracht seiner Relevanz im Rahmen des hard problem bedarf der Qualia-Begriff deshalb

\footnotetext{
143 Ebd.

144 A.a.O., S. 244 [Hervorhebung E.E.].
} 
dringend einer Revision, die der Vielfalt menschlichen Erlebens und dem damit verbundenen Desiderat phänomenaler Adäquatheit gerecht wird.

\subsubsection{Reichhaltigkeit und Heterogenität der Exterozeption}

Bereits auf der in der Qualia-Diskussion noch berücksichtigten Ebene exterozeptiver Sinneswahrnehmung, d.h. der sensorischen Wahrnehmung der Außenwelt, lässt sich eine Lücke verschiedenster Bereiche phänomenalen Erlebens feststellen. Allein von den zahlreichen Modalitäten der phänomenalen Kategorie der Exterozeption, werden meistens nur sehr wenige Standardbeispiele wie Schmerz ${ }^{145}$ oder »monosensuale Wahrnehmungsschemata «146, meistens anhand von Farbwahrnehmungen oder im Sinne einfachster Erscheinungsformen ${ }^{147}$ phänomenalen Erlebens erörtert. ${ }^{148}$

Nur sehr selten werden auditive, ${ }^{149}$ olfaktorische, ${ }^{150}$ oder gustatorische ${ }^{151}$ Sinnesmodalitäten als Fallbeispiele in Erwägung gezogen. Auch findet man fast

145 Vgl. z.B. Pitt, David:»The Phenomenology of Cognition or what is it Like to Think that P?«, Philosophy and Phenomenological Research, [2004] Vol. 69, No. 1, S. 30. Vgl. ebenfalls Lanz, Peter: Das phänomenale Bewusstsein. Eine Verteidigung, Frankfurt am Main: Klostermann 1996, S. 201.

146 Lorenz, Kuno: »Qualia«, in: Enzyklopädie Philosophie und Wissenschaftstheorie. Band 3: $P$-So, Mittelstrass, Jürgen [Hrsg.], Stuttgart: Metzler 2004, S. 426.

147 Vgl. Metzinger, Thomas [Hrsg.]: Grundkurs Philosophie des Geistes. Band 1: Phänomenales Bewusstsein, Paderborn: Mentis 2007, S. 57.

148 Beispiele zum herkömmlichen, zumeist monosensualen Verständnis von Qualia finden sich bereits in Abschnitt 8.1.

149 Obwohl auditive, analog zu visuellen Erlebnisqualitäten, als relativ einfache monosensuale Qualia angesehen werden können, weist Chalmers darauf hin, dass auditives für ihn noch seltsamer und rätselhafter ist als visuelles Erleben: »In some ways, sounds are even stranger than visual images. The structure of images usually corresponds to the structure of the world [...] but sounds can seem quite independent. My telephone receives an incoming call, an internal device vibrates, a complex wave is set up in the air and eventually reaches my eardrum, and somehow, almost magically, I hear a ring. Nothing about the quality of the ring seems to correspond directly to any structure in the world, although I certainly know that it originated with the speaker [...]. But why should that waveform, or even these neural firings, have given rise to a sound quality like that?«. Chalmers, David J: The conscious mind. In search of a fundamental theory, New York: Oxford University Press 1996, S. 7 [Hervorhebung im Original].

$15^{\circ}$ Vgl. Horgan, Terence: »Jackson on Physical Information and Qualia«, The Philosophical Quarterly, [1984] Vol. 34, No. 135, S. 147. Oder: Lorenz, Kuno: »Qualia«, in: Enzyklopädie Philosophie und Wissenschaftstheorie. Band 3: P-So, Mittelstrass, Jürgen [Hrsg.], Stuttgart: Metzler 2004, S. 426. Oder: Roth, Gerhard: Das Gehirn und seine Wirklichkeit. Kognitive Neurobiologie und ihre philosophischen Konsequenzen, Frankfurt am Main: Suhrkamp 1994, S. 22 und 104.

151 Vgl. bspw. Nagel, Thomas: Was bedeutet das alles? Eine ganz kurze Einführung in die Philosophie, Stuttgart: Reclam 2012, S. 33-34. 
nirgends Analysen, die sich mit dem Gleichgewichtssinn, dem Temperatursinn oder dem Tastsinn, ${ }^{152}$ beschäftigen, obwohl diese Sinne, genauso wie der Sehsinn, zum Katalog unserer sinnesphysiologischen Ausstattung und den mit ihnen verbundenen qualitativen Sinneserlebnissen gehören. ${ }^{153}$

Der Gleichgewichts- bzw. Raumorientierungs- und Bewegungssinn leistet einen wichtigen Beitrag zur Wahrnehmung der Position und Bewegungen unseres Körpers. Ein mit ihm verbundenes qualitatives Erleben kann auch in physiologisch oder psychologisch bedingten Schwindelgefühlen bestehen. Der Gleichgewichtssinn besteht aus insgesamt zehn Vestibularorganen. Die fünf wichtigsten Vestibularorgane befinden sich im Labyrinth des Innenohrs. ${ }^{154}$ Der Temperatursinn gliedert sich physiologisch in Warm- und Kaltsensoren, die an unterschiedlichen Hautpunkten wahrgenommen werden. Die wichtigste Region des Temperatursinnes ist die periorale Region, d.i. die Haut der Mundund Wangenregion. In der Haut der übrigen Körperregionen sind Warm- und Kaltpunkte bzw. -Sensoren jedoch nur spärlich vorhanden. Aus diesem Grund können wir kleinflächige Temperaturreize viel weniger gut differenzieren als grossflächige. ${ }^{155}$ Die phänomenale Modalität von Wärmeempfindungen kann auch, wie uns die eigene Erfahrung zeigt, auch im Zusammenhang mit Emotionen, wie Wut, Ärger oder Stress auftreten.

Sowohl der Tastsinn als auch die Mechanorezeption (resp. Mechanozeption) der Körperoberfläche bestehen in der Wahrnehmung von mechanischen

$15^{2}$ Chalmers bildet hier eine seltene Ausnahme: »Textures provide another of the richest quality spaces that we experience: think of the feel of velvet, and contrast it to the texture of cold metal, or a clammy hand, or a stubbly chin. All of these have their own unique quality. The tactile experiences of water, of cotton candy, or of another person's lips are different again«. Chalmers, David J.: The conscious mind. In search of a fundamental theory, New York: Oxford University Press 1996, S. 8.

153 Vgl. Schmidt, Robert F. [Hrsg.]: Neuro- und Sinnesphysiologie, Berlin: Springer 1998. Vgl. ebenfalls Birbaumer, Niels / Schmidt, Robert F.: Biologische Psychologie, Berlin: Springer 2003 .

154 Das Vestibular-Organ wird zwar als interozeptiver Sinn bezeichnet, weil es als Bewegungssinn einen wichtigen Beitrag zur Propriozeption, der Sensorik des eigenen Bewegungsapparates und der Wahrnehmung der Position unseres Körpers leistet. Da der Gleichgewichtssinn eine Empfindung über die Position des Körpers im Verhältnis zur Umwelt ist, könnte er m.E. aber mindestens in seiner Funktion als Raumorientierungsund Bewegungssinn auch der Exterozeption zugeordnet werden. Vgl. dazu Zenner, HansPeter: »Gleichgewicht«, in: Neuro- und Sinnesphysiologie, Schmidt, Robert F. [Hrsg.], Berlin: Springer 1998, S. 232, 329 und 33o. Vgl. auch Roth, Gerhard: Das Gehirn und seine Wirklichkeit. Kognitive Neurobiologie und ihre philosophischen Konsequenzen, Frankfurt am Main: Suhrkamp 1994, S. 322.

155 Vgl. Handwerker, Hermann:»Somatosensorik«, in: Neuro- und Sinnesphysiologie, Schmidt, Robert F. [Hrsg.], Berlin: Springer 1998, S. 233-234. 
Reizen, die auf die Haut einwirken. Das für den Tastsinn wichtigste Organ ist die Haut an der Innen- bzw. Palmarseite der Hand, wobei sich das stärkste Auflösungsvermögen des Tastsinnes an den Fingerspitzen findet. Daher trägt der Tastsinn im engeren Sinne bzw. der Tastsinn der Hände vor allem zur Gestaltwahrnehmung von Gegenständen bei (haptische Wahrnehmung). Dem Tastsinn liegen Mechanosensoren zu Grunde, mit welchen die Haut ausgestattet ist. ${ }^{156}$ Die Mechanorezeption der Körperoberfläche beschränkt sich jedoch weder auf den in der Diskussion schon kaum erwähnten Tastsinn der Hände noch auf einen Berührungssinn auf der ganzen Körperoberfläche. ${ }^{157}$ Nach Birbaumer lassen sich mit systematischen Untersuchungen allein bei der Mechanorezeption vier voneinander sich deutlich unterscheidende Qualitäten von Empfindungen nachweisen: Druck- Berührungs- Vibrations- und Kitzelempfindungen. ${ }^{158}$

Die hier erläuterten Beispiele kaum erwähnter Sinnesmodalitäten zeigen auf, dass die herkömmliche Verwendungsweise des Qualia-Begriffs allein schon in dieser Kategorie dem Desiderat phänomenaler Adäquatheit nicht standhält. Zudem erweist sich die häufig vorkommende Beschränkung auf monosensuale Schemata von Erleben als phänomenal inadäquat. Denn ein monosensualhomogenes und insofern auch momenthaftes Qualia-Verständnis widerspricht beispielsweise dem diachron-heterogenen phänomenalen Erleben einer Bach Kantate, ${ }^{159}$ die mit einer integrierten komplexen Fülle mannigfaltiger

156 Vgl. dazu a.a.O., S. 221 und 230.

157 Auch Körperregionen, die sich kaum zum Betasten von Objekten eignen, sind mit Mechanosensoren ausgestattet, die ganz ähnlich wie diejenigen der Handfläche sind. So liegen der Hautsensibilität der Körperoberfläche (auch Oberflächensensibilität genannt) ebenfalls Mechanosensoren der Haut zu Grunde. Die Mechanozeption dient infolgedessen nicht nur dem Tastsinn im engeren Sinne sondern bspw. auch der Wahrnehmung in den Füssen über die Beschaffenheit des Bodens oder das Empfinden eines bestimmten Kleidungsstückes am Rücken. Vgl. dazu ebd. Vgl. ebenfalls Boss, Norbert: Roche-Lexikon Medizin, München: Urban \& Schwarzenberg 1993, S. 1067.

$15^{8}$ Vgl. Birbaumer, Niels / Schmidt, Robert F.: Biologische Psychologie, Berlin: Springer 2003, S. 328.

159 Vgl. dazu Jung, Matthias / Heilinger, Jan-Christoph [Hrsg.]: Funktionen des Erlebens. Neue Perspektiven des qualitativen Bewusstseins. Band 5: Humanprojekt, Berlin: De Gruyter 2009, S. 9-11. So auch Chalmers: »Musical experience is perhaps the richest aspect of auditory experience, although the experience of speech must be close. Music is capable of washing over and completely absorbing us, surrounding us in a way that a visual field can surround us but in which auditory experiences usually do not. One can analyze aspects of musical experience by breaking the sounds we perceive into notes and tones with complex interrelationships, but the experience of music somehow goes beyond this. A unified qualitative experience arises [...] «. Chalmers, David J.: The conscious mind. In search of a fundamental theory, New York: Oxford University Press 1996, S. 7 . 
Erlebnisqualitäten, wie dem Spektrum reichhaltiger Klangerlebnisse, einer damit einhergehenden Stimmung, eines dabei vorherrschenden emotional gefärbten Denkmodus' und damit aufsteigenden visuellen Vorstellungen erlebt werden kann. ${ }^{160}$ Wenn also nur von isolierten, homogen-atomistischen (Farb-) Qualia gesprochen wird, so verfälscht dies schon auf der einfachen Ebene herkömmlicher Sinnesqualia mindestens in zweifacher Hinsicht phänomenales Erleben: 1.) Der diachrone Kontext von Erlebens wird nicht berücksichtigt; 2.) die Integration einer komplexen Fülle von Erlebnisqualitäten in Form von »multisensualen Schemata «161 und »intersensuale Schemata «162 wird ignoriert. ${ }^{163}$ Aus Platzgründen werde ich auf diese, im Vergleich zu weiteren Mängeln des traditionellen Qualia-Begriffs, weniger schwerwiegende Lücke aber nicht weiter eingehen.

\subsubsection{Interozeption, somatoviszerale Sensibilität und Emotion}

Im Zusammenhang mit meiner Arbeitshypothese über den phänomenalen Charakter von Emotionen lässt sich des Weiteren kritisieren, dass mit einem traditionellen Qualia-Begriff, neben den vernachlässigten Sinnesmodalitäten der Exterozeption, die komplexe Fülle interozeptiver ${ }^{164}$ Erlebnisqualitäten mit Ausnahme der üblichen Beispiele nozizeptiver Qualia (Schmerz) - noch viel weniger berücksichtigt werden. Doch ein phänomenal angemessenes Qualia-Verständnis hat körperlich basierte Wahrnehmungserlebnisse der Interozeption aufgrund ihres Zusammenhangs mit emotionalem Erleben unbedingt mit einzuschliessen. $\mathrm{Zu}$ den interozeptiven Erlebnisqualitäten lassen

16o Mehr über Emotionen und emotive Formen des Denkens, die ebenfalls eine Weise phänomenalen Erlebens darstellen, vgl. Abschnitte 8.2.3 und 8.2.4.

161 So verweist Lorenz darauf, dass zu den Qualia auch »multisensuale Schemata«, wie z.B. "gross«, »aufrecht«, »schwer « gehören (solange auf eine intersubjektive Schematisierung verzichtet werde). Oder mit Chalmers lässt sich beispielsweise auf eng verwobene Geruchs- und Geschmacksempfindungen verweisen, die, wenn wir noch Wärmeempfindungen und das Spüren der Konsistenz des Essens berücksichtigen, zu polydimensionalen Sinneserlebnissen werden können. Vgl. dazu a.a.O., S. 10.

162 »Intersensuale Schemata « werden Lorenz zufolge in Handlungen mit Gegenständen erlebt: z.B »auf Bäume Klettern« oder »Ballwerfen«. Vgl. Lorenz, Kuno: »Qualia«, in: Enzyklopädie Philosophie und Wissenschaftstheorie. Band 3: P-So, Mittelstrass, Jürgen [Hrsg.], Stuttgart: Metzler 2004, S. 426.

163 Vgl. dazu Jung, Matthias / Heilinger, Jan-Christoph [Hrsg.]: Funktionen des Erlebens. Neue Perspektiven des qualitativen Bewusstseins. Band 5: Humanprojekt, Berlin: De Gruyter 2009, S. 10-11.

164 Unter Interozeption versteht man die Wahrnehmung von Reizen aus dem Körperinneren, im Gegensatz zum exterozeptiven Wahrnehmen optischer, thermischer, akustischer, olfaktorischer etc. Reize der Aussenwelt. 
sich die meisten ${ }^{165}$ somatosensorischen bzw. alle Arten von propriozeptiven und viszeralen Empfindungen zählen.

Als somatosensorisch bezeichnet man alle Empfindungen, die durch die Reizung der verschiedensten Sensoren unseres Körpers hervorgerufen werden können. Davon ausgenommen sind aber die so genannten »spezifischen« in der Qualia-Diskussion zumeist berücksichtigten Sinnesorgane, die im Kopf lokalisiert sind. ${ }^{166}$ Auch der auf Nozizeption basierende und in der QualiaDiskussion oft erwähnte Schmerz wird zu Somatosensorik gerechnet. ${ }^{167}$ Die Gesamtheit der somatosensorischen Empfindungen wird auch als somatoviszerale Sensibilität bezeichnet. ${ }^{168}$

Unter Propriozeption versteht man alle somatischen Empfindungen, die durch die Reizung von Muskeln, Sehnen- und Gelenkmechanosensoren hervorgerufen werden. Mit dieser Sinnesmodalität nehmen wir die Stellung (Positionssinn) und Bewegungen (Kinästhesie) einzelner Körperteile bzw. des ganzen Körpers wahr (z.B. unser Körpergefühl beim Tanzen oder Klettern). ${ }^{169}$

Die Viszerozeption (bzw. eine viszerale Empfindung) basiert auf bewusst wahrgenommenen Afferenzen von inneren Organen. Die meisten dieser Afferenzen führen jedoch nicht zu bewussten Empfindungen, sondern dienen reflektorischen Regelungen. ${ }^{170}$ Dennoch führen Afferenzen aus inneren

165 Die Hautsensibilität (bzw. Oberflächensensibilität), die ebenfalls dem Bereich der somatosensorischen Empfindungen zugerechnet wird, lässt sich jedoch eher den exterozeptiven Sinnesempfindungen zurechnen, da mit ihr bspw. Form, Vibration oder Temperatur von Gegenständen erfahren werden. Vgl. dazu Birbaumer, Niels / Schmidt, Robert F.: Biologische Psychologie, Berlin: Springer 2003, S. 328-335. Grob lässt sich die Hautsensibilität in einen mechanischen und einen thermischen Sinn einteilen. Vgl. dazu Boss, Norbert: Roche-Lexikon Medizin, München: Urban \& Schwarzenberg 1993, S. 1211. Vgl. auch Handwerker, Hermann: »Somatosensorik«, in: Neuro- und Sinnesphysiologie, Schmidt, Robert F. [Hrsg.], Berlin: Springer 1998, S. 221-236.

166 Zu den »>spezifischen`Sinnesorganen« zählt Handwerker den Seh-, Hör-, Geschmacks-, Geruchs- und den Gleichgewichtssinn. Vgl. a.a.O., S. 221.

167 Vgl. a.a.O., S. 221 und 249-261.

168 Die Gesamtheit der Somatosensorik umfasst nach Hermann Handwerker drei Bereiche: die Sensorik der Körperoberfläche bzw. die Hautsensibilität, die Sensorik des Bewegungsapparates, bzw. die Propriozeption und die Sensorik der inneren Organe, auch Viszerozeption genannt. Vgl. a.a.O., S. 221.

Die Viszerozeption wird zuweilen auch, wie von Handwerker, Enterozeption genannt. Aufgrund der (verwirrenden) Nähe dieses Begriffs zum Begriff Interozeption, verzichte ich jedoch in dieser Arbeit auf diese Bezeichnung. Vgl. dazu a.a.O., S. 221 und 237.

169 Vgl. a.a.O., S. 232.

170 Ein Beispiel für solche unbewussten Afferenzen aus inneren Organen sind die Chemosensoren der Leber, die unter anderem den Glukosespiegel des Blutes registrieren. Vgl. a.a.O., S. 238. Auch tragen viszerale Empfindungen nach Handwerker in der Regel eher zu unbestimmten Gefühlen bei, als zu klar lokalisierbaren Empfindungen. Nach ihm hängt 
Organen, auch zu bewussten viszeralen Empfindungen, die charakteristisch bei emotionaler Aufgeregtheit sind. Viszerale Körpersignale können bspw. als positive oder negative so genannte "gut feelings «171 oder als Herzrasen auftreten. Körpersignale aus dem Magen, können auch als >Magenwürgen<, $>$ Kribbeln<, >Rumoren $<$ oder gar als $>$ Schmetterlinge im Bauch $<$ erlebt werden. Die Fülle interozeptiver Qualia-Kategorien ist für phänomenales Erleben von entscheidender Relevanz. Neben der Wichtigkeit für das Körpergefühl und Ekelempfindungen ${ }^{172}$ spielen interozeptive Körperwahrnehmungen, so die These physiologisch basierter Emotionstheorien nach James-Lange, auch beim Erleben von Emotionen eine entscheidende Rolle. ${ }^{173}$ Dass somatische Empfindungen eine entscheidende Rolle bei Emotionen spielen, wird auch durch neurobiologische Ansätzen abgestützt. ${ }^{174}$ Obwohl nicht unumstritten

das damit zusammen, dass von den inneren Organen viel weniger Afferenzen ins Zentralnervensystem gelangen als bspw. von der Haut. Ebd.

171 Jung, Matthias / Heilinger, Jan-Christoph [Hrsg.]: Funktionen des Erlebens. Neue Perspektiven des qualitativen Bewusstseins. Band 5: Humanprojekt, Berlin: De Gruyter 2009, S. 19. Ebenfalls Damasio, Antonio R.: Descartes' error. Emotion, reason and the human brain, London: Vintage [zuerst 1994]/2006, S. 173.

172 Selbstverständlich können Ekelempfindungen auch als Emotionen gesehen werden oder diese begleiten. Ekel wird trotz (oder gerade wegen) seiner starken somatischen Komponente von Emotionsforschern wie bspw. Paul Ekman als Grundemotion aufgefasst. So geht Ekman von folgenden sechs Grundemotionen aus, die angeblich kulturinvariant sind und auf der ganzen Welt mimisch gleich ausgedrückt werden: Angst, Ekel, Trauer, Ärger, Überraschung und Freude. Vgl. Wassmann, Claudia: Die Macht der Emotionen. Wie Gefühle unser Denken und Handeln beeinflussen, Darmstadt: Primus Verlag 2002, S. 35-37. Analog zu Ciompi, der Müdigkeit, Hunger und Durst als psycho-physische Befindlichkeiten betrachtet, die nicht identisch mit Emotionen sein müssen, betrachte ich Ekelempfindungen jedoch nicht per se als Emotionen. Vgl. dazu Ciompi, Luc: Die emotionalen Grundlagen des Denkens. Entwurf einer fraktalen Affektlogik, Göttingen: Vandenhoeck \& Ruprecht 1997, S. 68.

173 Vereinfacht gesagt, gehen Emotionstheorien nach James-Lange davon aus, dass Gefühle unauflöslich an körperliche Vorgänge gebunden sind. Vgl. dazu James, William: »What Is an Emotion?«, Mind, [1884] Vol. 9, No. 34. Vgl. auch Lange, Carl G. / Kurella, Hans: Ueber Gemüthsbewegungen. Eine psycho-physiologische Studie, Leipzig: T. Thomas 1887. Vgl. ebenfalls LeDoux, Joseph E.: Das Netz der Gefühle. Wie Emotionen entstehen, München \& Wien: Hanser 1998, S. 48 und 5o.

174 Vgl. z.B. Prinz, Jesse: »Are Emotions Feelings?«, Journal of Consciousness Studies, [2005] Vol. 12, No. 8-10, S. 13. Oder: Damasio, Antonio R.: Descartes' error. Emotion, reason and the human brain, London: Vintage [zuerst 1994]/2006. Oder: Critchley, Hugo D. / Wiens, Stefan / Rotshtein, Pia, et al.: »Neural systems supporting interoceptive awareness«, Nature Neuroscience, [2004] Vol. 7, No. 2, S. 189-195. Oder: Damasio, Antonio R.: Looking for Spinoza. Joy, sorrow and the feeling brain, London: Vintage 2004. Oder: Damasio, Antonio R.: The feeling of what happens. Body, emotion and the making of consciousness, London: Vintage 200o. Oder: LeDoux, Joseph E.: Das Netz der Gefühle. Wie Emotionen entstehen, München \& Wien: Hanser 1998. Oder: Shin, Lisa M. / Darin D. Dougherty / 
ist, inwieweit Körperempfindungen für Emotionen konstitutiv sind ${ }^{175}$ oder ob es nicht doch so etwas wie »entkörperte « ${ }^{176}$ Emotionen gibt, lässt sich feststellen, dass interozeptiven Wahrnehmungen, die mit physiologischen Veränderungen im Körper zusammenhängen, im Hinblick auf das phänomenale Erleben von Emotionen eine zentrale Bedeutung zukommen. ${ }^{177}$

Scott P. Orr, et al.: »Activation of Anterior Paralimbic Structures during Guilt-Related Script-Driven Imagery«, Biological Psychiatry, [200o] Vol. 48, No. 1, S.43-50. Oder: Antonio R. Damasio / Thomas J. Grabowski / Antoine Bechara, et al.: »Subcortical and cortical brain activity during the feeling of self-generated emotions «, Nature Neuroscience, [2000] Vol. 3, No. 10, S. 1049-1056. Oder: Joshua D. Greene / Sommerville, Brian R. / Leigh, Nystrom E., et al.: »An fMRI Investigation of Emotional Engagement in Moral Judgment«, Science (New Series), [2001] Vol. 293, No. 5537, S. 2105-2108.

175 Vgl. z.B. Leighton, Stephen R.: »A New View of Emotion«, American Philosophical Quarterly, [1985] Vol. 22, No. 2, S. 134. Oder: Pugmire, David: Rediscovering emotion, Edinburgh: Edinburgh University Press 1998, S. 8, 14 und 95. Oder: Solomon, Robert C.: The passions, Notre Dame, Indiana: University of Notre Dame Press [zuerst 1976]/1983. Oder: Goldie, Peter: »Emotionen und Gefühle«, in: Philosophie der Gefühle, Döring, Sabine [Hrsg.], Frankfurt am Main: Suhrkamp [zuerst 20o2, engl.]/20o9, S. 372-373. Oder: Stocker, Michael: »Psychic feelings their importance and irreducibility«, Australasian Journal of Philosophy, [1983] Vol. 61, No. 1, S. 5 und 19-20. Oder: Harré, Rom: The social construction of emotions, Oxford: Basil Blackwell 1986. Oder: Griffiths, Paul E.: What emotions really are. The problem of psychological categories, Chicago: University of Chicago Press 1997.Vgl. auch Engelen, die feststellt, dass bei Versuchspersonen physische Reaktionen ausgelöst werden können, die normalerweise mit Emotionen, z.B. mit Angst einhergehen, ohne dass die Personen jedoch Angst verspüren würden. Engelen, Eva-Maria: Gefühle, Stuttgart: Reclam 2007, S. 25. Zum Pro- und Kontra-Lager des James-Lange Ansatz, vgl. auch Slaby, Jan: »James: Von der Physiologie zur Phänomenologie«, in: Klassische Emotionstheorien. Von Platon bis Wittgenstein, Landweer, Hilge / Renz, Ursula [Hrsg.], Berlin: De Gruyter [zuerst 2008]/2012, S. 549 .

176 So postuliert Stocker im Zusammenhang mit seiner Konzeption der »psychic feelings« auch körperlose Gefühle (»nonbodily feelings«): »[...] various feelings do not seem to require any bodily feeling [...] «. Stocker, Michael / Hegeman, Elizabeth: Valuing emotions, Cambridge, Massachusetts: Cambridge University Press 20o1, S. 19. Doch selbst Stocker räumt ein, dass der Körper bei Emotionen eine zentrale Rolle spielen kann: »Some emotions and feelings are most naturally, and perhaps even necessarily, expressed by bodily means. Further, various feelings seem at once bodily and psychic [...]«. Ebd.

177 Dies scheint insbesondere bei intensivem emotionalen Erleben der Fall zu sein: es ist irgendwie ein ängstlich-beklemmendes Stechen oder wuterfüllten, schmerzenden Druck in der Brust zu empfinden. Trotz seiner Kritik an der Konzeption, dass alle Emotionen bzw. Gefühle von einer körperlichen Empfindungen begleitet sein müssen, stellt auch Stocker fest, dass wir, um bspw. eine Emotion der Wut zu haben, nicht nur bestimmte evaluative Gedanken und Wünsche haben müssen, sondern auch eine bestimmte mit diesen wertenden Gedanken einhergehende unangenehme Körperempfindung. A.a.O., S. 25 .

Im Zusammenhang mit dem Einwand von Kritikern der James-Lange-Theorien, dass nur bestimmte, vorwiegend basale bzw. phylogenetisch weniger entwickelte Emotionen 
Wie auch für William James ${ }^{178}$ sind für die neurobiologischen Ansätze von Damasio und LeDoux, im Gegensatz zu Stocker, ${ }^{179}$ keine »körperlosen Emotionen ${ }^{180}$ vorstellbar. ${ }^{181}$ Für LeDoux sind körperliche Reaktionen bzw. emotionsspezifische propriozeptive und viszerale Rückmeldungsmuster »[...] ein integraler Bestandteil des gesamten emotionalen Prozesses «. ${ }^{182}$ Nach ihm ergeben sich aus den einzelnen an emotionalem Erleben beteiligten Systemen ${ }^{183}$ »atemberaubende Möglichkeiten « ${ }^{184}$ für die Erzeugung emotionsspezifischer Rückmeldungsmuster; wobei den körperlichen Rückmeldungsmuster eine

mit körperlichen Veränderungen auftreten, dass es auch empirische Belege für körperliche Veränderungen bei subtileren Emotionen (»more advanced emotion[s] «) gibt. Vgl. Prinz, Jesse: »Are Emotions Feelings?«, Journal of Consciousness Studies, [2005] Vol. 12, No. 8-10, S. 13. Über die Frage, zur Rolle von körperlichen Empfindungen bei Emotionen vgl. auch Hartmann, Martin: Gefühle. Wie die Wissenschaften sie erklären, Frankfurt am Main: Campus 2010, S. 98-100.

178 So konstatiert W. James: »A purely disembodied human emotion is a nonentity«. James, William: »What Is an Emotion?«, Mind, [1884] Vol. 9, No. 34, S. 194. Vgl. dazu auch LeDoux, Joseph E.: Das Netz der Gefühle. Wie Emotionen entstehen, München \& Wien: Hanser 1998, S. 45-46. Zu James, vgl. auch Hartmann, Martin: Gefühle. Wie die Wissenschaften sie erklären, Frankfurt am Main: Campus 2010, S. 40.

179 Vgl. Stocker, Michael / Hegeman, Elizabeth: Valuing emotions, Cambridge, Massachusetts: Cambridge University Press 2001, S. 19.

180 LeDoux, Joseph E.: Das Netz der Gefühle. Wie Emotionen entstehen, München \& Wien: Hanser 1998, S. 317. Vgl. ebenfalls Damasio, Antonio R.: The feeling of what happens. Body, emotion and the making of consciousness, London: Vintage 200o, S. 293.

181 Vgl. dazu auch a.a.O., S. 284. Vgl. ebenfalls Damasio, Antonio R.: Descartes' error. Emotion, reason and the human brain, London: Vintage [zuerst 1994]/2006, S. 250-252.

182 LeDoux, Joseph E.: Das Netz der Gefühle. Wie Emotionen entstehen, München \& Wien: Hanser 1998, S. 46.

183 Nach LeDoux konstituieren sich emotionale Erlebnisse durch die Aktivität folgender Systemen: 1.) Ein spezialisiertes Emotionssystem welches Reaktionen des autonomen Nervensystems und des Verhaltens hervorruft, wie Veränderungen des Blutdrucks, Herzfrequenz, Schwitzen, Piloarrektion sowie hormonale Reaktionen wie z.B. die Ausschüttung von Adrenalin oder Nebennierensteroiden. 2.) Kortikale sensorische Puffer, welche bestimmte Informationen über einen aktuell gegebenen Sinnesreiz festhalten. 3.) Das Arbeitsgedächtnis, welches Informationen, die für die Emotion relevant sind aus dem Langzeitgedächtnis abruft. 4.) Eine kortikale Erregung. 5.) Somatische (propriozeptorische) und viszerale Informationen, die während einer emotionalen Reaktion auftreten. Wenn all diese Systeme aktiviert sind, sei ein bewusstes emotionales Erleben unvermeidlich. Wenn nur einige dieser Systemen aktiviert sind kann es jedoch, je nachdem um welche Systeme es sich handelt, zu einem emotionalen Erlebnis kommen (so gibt es z.B. ohne die Aktivierung der Amygdala für LeDoux kein vollständiges Gefühl der Furcht, jedoch kann man ein emotionales Erlebnis haben ohne dass im Arbeitsgedächtnis der Reiz festgehalten ist bzw. ohne dass man sich des auslösenden Reizes bewusst sei). A.a.O., S. 313-321.

184 A.a.O., S. 315 . 
zentrale Rolle zukommt: »[...] ich sehe nicht viele Möglichkeiten, wie ein vollblütiges emotionales Gefühl existieren könnte ohne einen Körper, der an dem Gehirn hängt $[\ldots] \ll .185$ Demnach spielen die propriozeptiven Rückmeldungen der Gesichtsmuskulatur oder das gesamte Empfindungsmuster somatischer und viszeraler Rückmeldungen des Körpers im Zusammenhang mit emotionalen Erlebnissen eine entscheidende Rolle. ${ }^{186}$ Natürlich spielen auch spezifische Muster chemischer Rückmeldungen von inneren Organen, wie die Ausschüttung von Steroid- oder Peptidhormonen, bei emotionaler Erregung eine Rolle. Diese Muster von Hormonausschüttungen gelangen mit dem Blutstrom zum Gehirn. Die Übergänge von einer Emotion zu einer anderen könnten nach LeDoux durch die Aktivierung verschiedener Emotionssysteme, insbesondere durch diese spezifischen chemischen Rückmeldungsmuster der inneren Organe hervorgerufen werden. ${ }^{187}$ So können weder die Rückmeldungen der viszeralen Organe noch die von den inneren Organen ausgeschütteten Hormone der einzige Faktor sein, der die Spezifität und Dynamik des emotionalen Erlebens ausmacht. Denn sowohl die Rückmeldungen der »glatten «, träge reagierenden Muskeln der viszeralen Organe als auch die Rückmeldungsmuster von Hormonen, bei welchen es Stunden dauern kann, bis sie sich im Gehirn auswirken, sind zu langsam. ${ }^{188}$

Folglich sind nicht nur viszerale, sondern auch propriozeptive Rückmeldungsmuster für das emotionale Erleben wichtig. Denn die gestreiften Muskeln der Gesichtsmuskulatur könnten demgegenüber die nötige Geschwindigkeit und Spezifität besitzen: es dauert weniger als eine Sekunde bis sie auf einen Reiz reagiert haben. ${ }^{189}$ Die meisten Emotionstheoretiker, die von einem Zusammenhang zwischen emotionalem Erleben und somatischen

185 A.a.O., S. 318. Allerdings sollten hier nach LeDoux die von Damasio bezeichneten »Alsob-Schleifen« nicht unerwähnt bleiben: Unter bestimmten Umständen ist es möglich, sich nur vorzustellen, wie sich eine körperliche Rückmeldung anfühlen würde. Allein die Vorstellung einer körperlichen Rückmeldung kann Emotionen beeinflussen. Beispielsweise haben LeDoux zufolge die blossen Vorstellungen von Versuchspersonen, dass sich ihre Herzfrequenz angeblich geändert habe, sie dazu veranlasst, zu empfinden, sie seien emotional erregt. Hier muss jedoch eingewendet werden, dass eine solche vorgestellte körperliche Rückmeldung wiederum nur möglich ist, wenn jemand schon viele reale körperliche Rückmeldungen erlebt hat. Das Phänomen der »Als-ob-Schleifen« spricht also eher für die wichtige Rolle körperlicher Empfindungen bzw. Rückmeldungsmuster, als dass es dagegen spricht. A.a.O., S. 317-318.

186 A.a.O., S. 315 .

187 A.a.O., S. 314-315.

188 Ebd.

189 A.a.O., S. 315 . 
Rückmeldungsmustern ausgehen, verorten diesen nach LeDoux deshalb in den Rückmeldungsmustern der Gesichtsmuskulatur. ${ }^{190}$

Nach Goldie besteht die somatische Basis für Emotionen wiederum nicht nur in viszeralen bzw. propriozeptiven Körpergefühlen, sondern auch in »erweiterten «191 Körperempfindungen der Oberflächensensibilität. Goldie schliesst auf derartige erweiterte Körpergefühle (»extension of bodily feelings «), ${ }^{192}$ weil die Hautsensibilität der Körperoberfläche durch den Kontakt mit bestimmten Objekten insofern erweitert ist, als wir, je nach emotionaler Verfassung, auch die von uns berührten Objekte anders erleben. So fühlen wir bspw. dass der Abzug eines Revolvers glitschig ist, was uns vielleicht erst dann bewusst macht, dass wir aufgeregt oder ängstlich sind. ${ }^{193}$

Auch Prinz zufolge spricht vieles dafür, dass emotionales Erleben auf der Wahrnehmung von sich ändernden körperlichen Zuständen beruht. ${ }^{194}$ Diese somatischen Veränderungsprozesse sind nach ihm nicht nur ein Effekt von Emotionen, sondern grundlegend für letztere. Unter Rückgriff auf James plädiert er dafür, dass das bewusste Erleben jener somatischen Veränderungen nicht nur ein Teil des Emotionalen ausmacht, sondern Emotionen konstituieren: »Most people assume that these changes are the effects of our emotions, but James argues that this is backwards. Our bodies change, and an emotion >just is the feeling of that change $[\ldots] \ll{ }^{195}$ Wenn wir uns vorstellen, dass wir alle somatischen Erscheinungen von einer Emotion nicht fühlen

190 Ebd. Für diese These spricht zwar der Umstand, dass Personen mit Rückenmarkverletzungen, trotz schwerer Schädigung des Informationsflusses zwischen somatischen Erregungsmustern mit dem Zentralnervensystem, keine Defizite im emotionalen Erleben zeigen. Die Empfindungen der Bewegungen der Gesichtsmuskulatur verlaufen direkt vom Gesicht zum Gehirn und sind folglich bei Rückenmarksverletzungen nicht beeinträchtigt. Allerdings unterbrechen Rückenmarksverletzungen ohnehin nicht vollständig den Informationsfluss zwischen dem Gehirn und dem Restkörper. A.a.O., S. 316. LeDoux erwähnt auch, dass Ekman Versuchspersonen dazu brachte, bestimmte Gesichtsausdrücke zu zeigen, die für bestimmte Emotionen charakteristisch sind, ohne dass ihnen aber bewusst war um welche Emotionen es sich bei den Gesichtsausdrücken handelte. Anschliessend stellte sich durch Befragungen heraus, dass die Gefühle der Versuchspersonen erheblich von diesen Gesichtsbewegungen beeinflusst wurden. A.a.O., S. 317 .

191 Goldie, Peter: »Emotions, feelings and intentionality«, Phenomenology and the Cognitive Sciences, [2002] Vol. 1, No. 3, S. 236.

192 Ebd.

193 Vgl. dazu ebd. Für Goldie besteht die Erweiterung des Körpergefühls also darin, dass sich in diesem Beispiel nicht nur die Eigenschaft unserer Hautoberfläche sondern auch die Eigenschaft eines von uns berührten Objektes unserer Aufmerksamkeit durch den Schweiss verändert hat.

194 Vgl. Prinz, Jesse: »Are Emotions Feelings?«, Journal of Consciousness Studies, [2005] Vol. 12, No. 8-10, S. 12.

195 Ebd. 
würden, so wäre Prinz zufolge auch keine Emotion mehr spürbar. ${ }^{196}$ An diesem Argument, das auf William James ${ }^{197}$ zurückgeht, wird jedoch kritisiert, dass es auf einem Rückgriff auf Introspektion beruhe: »I think James's argument is compelling, but some critics are uncomfortable with arguments that depend on introspection «.198 Prinz stütz sich deshalb auf neurowissenschaftliche Studien im Zusammenhang mit interozeptiven Wahrnehmungssystemen, die darauf Hinweisen, dass Emotionen mit dem Erleben somatischer Veränderungsprozesse zusammenhängen. ${ }^{199}$

Schliesslich geht Damasio, im Zusammenhang mit seiner Hypothese der somatischen Marker ${ }^{200}$ davon aus, dass zwar sowohl die Rückmeldungsmuster viszeraler als auch propriozeptiver Empfindungen eine zentrale Rolle bei emotionalen Zuständen spielen, ${ }^{201}$ die viszeralen aber vermutlich wichtiger seien:

[...] the idea of the somatic marker encompasses an integral change of body state, which includes modifications in both the viscera and the musculoskeletal system, induced by both neural signals and chemical signals, although the visceral component seems somewhat more critical than the musculoskeletal in the construction of background and emotional states. ${ }^{202}$

Im Hinblick auf das Erleben emotionaler Zustände bzw. Gefühlen, ${ }^{203}$ sind die somatischen Rückmeldungsmuster der Viszerozeption und des

196 Vgl. ebd. Prinz räumt aber ein, dass es auch unbewusste mit Emotionen zusammenhängende somatische Veränderungen gebe: »Unconscious emotions are also possible, so not all emotions are feelings. Some emotions aren't felt«. A.a.O., S. 23.

197 Vgl. James, William:»What Is an Emotion?«, Mind, [1884] Vol. 9, No. 34, S. 190.

198 Prinz, Jesse: »Are Emotions Feelings?«, Journal of Consciousness Studies, [2005] Vol. 12, No. 8-10, S. 12.

199 Vgl. a.a.O., S. 13. Vgl. dazu auch die Studie von Critchley, Hugo D. / Wiens, Stefan / Rotshtein, Pia, et al.: »Neural systems supporting interoceptive awareness«, Nature Neuroscience, [2004] Vol. 7, No. 2, S. 189-195. Vgl. ebenfalls Antonio R. Damasio / Thomas J. Grabowski / Antoine Bechara, et al.: »Subcortical and cortical brain activity during the feeling of selfgenerated emotions«, Nature Neuroscience, [2000] Vol. 3, No. 10, S. 1049-1056.

200 Vgl. Damasio, Antonio R.: Descartes' error. Emotion, reason and the human brain, London: Vintage [zuerst 1994]/2006, S. 165-204. Über empirisch-experimentelle Indizien zur Stützung der Hypothese der somatischen Marker vgl. a.a.O., S. 205-222.

201 Vgl. a.a.O., S. 129-201. Vgl. dazu auch Damasio, Antonio R.: Looking for Spinoza. Joy, sorrow and the feeling brain, London: Vintage 2004, S. 89.

$2 \mathrm{O} 2$ Damasio, Antonio R.: Descartes' error. Emotion, reason and the human brain, London: Vintage [zuerst 1994]/2006, S. 206.

203 Wie in den Begriffsbestimmungen erwähnt, bezeichnen Damasio und Prinz das Erleben emotionaler Zustände als »feelings«, im Gegensatz zu den physiologischen bzw. öffentlich beobachtbaren affektiv-emotionalen Vorgängen, die sie »Emotions« 
musculoskeletalen Systems (Propriozeption) Damasio zufolge auch wichtig, weil sie eine Vorstellung oder Erinnerung »somatisch markieren $\ll^{204}$ Aus diesen Rückmeldungsmustern entsteht, so die Hypothese der somatischen Marker, ein »Bauchgefühl « (»gut feeling «) ${ }^{205}$ welches bei Entscheidungs- bzw. Bewertungsprozessen ein enorm wichtiger Faktor darstelle. ${ }^{206}$

In Situationen, in welchen man zuwenig Zeit habe, über eine Emotion, die man gerade erlebt, zu reflektieren, konstituiert sich, Damasio zufolge, emotionales Erleben sogar ausschliesslich aus der Wahrnehmung eines bestimmten somatoviszeralen Zustandes. ${ }^{207}$ Ansonsten ist nach ihm aber der ganze Komplex emotionalen Erlebens nicht nur mit somatoviszeralen Empfindungen verbunden, sondern auch mit Situationsbewertungen, bestimmten mit Emotionen korrelierenden Modi des Denkens und emotionsgeladenen

nennen. Vgl. Damasio, Antonio R.: Looking for Spinoza. Joy, sorrow and the feeling brain, London: Vintage 2004, S. 86. Vgl. auch Prinz, Jesse: »Are Emotions Feelings?«, Journal of Consciousness Studies, [2005] Vol. 12, No. 8-10, S. 9 und 23.

204 »Because the feeling is about the body, I gave the phenomenon the technical term somatic state [...] and because it >marks« an image, I called it a marker«. Damasio, Antonio R.: Descartes' error. Emotion, reason and the human brain, London: Vintage [zuerst 1994]/2006, S. 173 [Hervorhebung im Original].

205 Ebd.

206 In seiner Hypothese der somatischen Marker geht Damasio davon aus, dass diese bei bedeutenden persönlichen Entscheidungsprozessen eine zentrale Rolle spielen: noch bevor wir fähig seien, eine bestimmte Kosten-Nutzen-Analyse zu machen, entstünden neben Vorstellungen, die mit bestimmten Konsequenzen einer Handlungsoption zusammenhängen, auch »somatische« Zustände. Diese somatischen Zustände »markieren« eine solche Vorstellung. Insbesondere zusammen mit einer Vorstellung von ungünstigen Konsequenzen entstehe aus ihnen ein »Bauchgefühl« (»gut feeling«) welches unsere Aufmerksamkeit auf diese möglichen negativen Konsequenzen lenke und so die Anzahl der Möglichkeiten, die in Erwägung gezogen werden müssen, drastisch reduzieren. Die »somatischen Marker« erhöhen deshalb nach Damasio die Effizienz von Entscheidungsprozessen und besitzen auch die Funktion eines automatischen Warnsystems, welches uns sage: »Beware of danger ahead if you choose the option which leads to this outcome«. Ebd.

Zur Stützung dieser These führt Damasio Beispiele von Patienten mit Frontallappenschädigungen auf, die trotz ihrem unbeeinträchtigtem faktischen Wissen über eine gefahrenvolle oder kritische Situation keinen Zugang zu jenen »somatischen Marker« haben, die bei Gesunden als warnendes >Bauchgefühl die damit verbundenen Vorstellungen >markieren<. Aus diesem Grund haben Patienten, die diese »somatischen Marker« nicht erleben können, offenbar Mühe, negative Konsequenzen ihres Verhaltens abzuschätzen und danach zu handeln. A.a.O., S. 211 und 216-217.

207 So konstatiert Damasio: »In many circumstances, especially when there is little or no time to examine feelings, feelings are solely the perception of a certain body state «. Damasio, Antonio R.: Looking for Spinoza. Joy, sorrow and the feeling brain, London: Vintage 2004, S. 89 . 
Vorstellungen bzw. Erinnerungen. ${ }^{208}$ Auf diese Interdependenz von emotionalem Erleben und kognitiven Verarbeitungsprozessen gehe ich weiter unten ein (vgl. Abschnitt 8.2.4).

Trotz aller Kritik am James-Lange-Ansatz zeigen die hier aufgeführten Positionen auf, dass somatische Empfindungen eine entscheidende Rolle bei emotionalem Erleben spielen und dass ein fundamentaler Zusammenhang zwischen emotionalem Erleben und bewusst wahrgenommenen somatoviszeralen Zuständen besteht. ${ }^{209}$ Dies gilt auch dann, wenn Emotionen, wie ich weiter unten ausführen werde, mit kognitiven Prozessen stark zusammenhängen. Schmerz als typisches Beispiel einer körperbasierten bzw. somatoviszeralen Empfindungsqualität bildet einen Standardfall von Qualia. Somit müssen auch die, bei emotionalem Erleben zentralen somatoviszeralen Empfindungen, im Katalog phänomenalen Erlebens mitberücksichtig werden, wenn wir mit einem phänomenal adäquaten Qualia-Begriff arbeiten wollen. Auf dieser Grundlage werde ich im nächsten Abschnitt näher darauf eingehen, warum das bewusste Erleben von Emotionen als phänomenales Erleben bzw. als eine weitere, noch kaum diskutierte Kategorie von Qualia verstanden werden sollte.

\subsubsection{E-Qualia: Emotiv-phänomenales Erleben}

Emotionen wie Wut, Liebe, Neid, Eifersucht, Trauer, Euphorie, Angst etc. können allein schon aufgrund ihres wie-es-ist-Charakters als »Zustände der phänomenalen Erfahrung « ${ }^{210}$ oder als phänomenale Qualitäten ${ }^{211}$ bezeichnet werden. So führt Chalmers in einer Aufzählung bewusster Erlebnisse, die für ihn mehr oder weniger synonym (»roughly synonymous«) $)^{212}$ mit Qualia

208 Vgl. Damasio, Antonio R.: Descartes' error. Emotion, reason and the human brain, London: Vintage [zuerst 1994]/2006, S. 146 und 224-225. Vgl. ebenfalls Damasio, Antonio R.: Looking for Spinoza. Joy, sorrow and the feeling brain, London: Vintage 2004, S. 89.

209 Selbst wenn es sich, wie beim Phänomen der »Als-ob-Schleifen«, nur um vorgestellte körperliche Rückmeldung handelt, so kann sich jemand im Zusammenhang mit dem Erleben einer Emotion diese Rückmeldungen nur vorstellen, wenn er oder sie zuvor schon einmal jene somatischen Empfindungen erlebt hat, die mit besagter Emotion einhergingen. Vgl. dazu auch LeDoux, Joseph E.: Das Netz der Gefühle. Wie Emotionen entstehen, München \& Wien: Hanser 1998, S. 317-318.

210 Döring, Sabine / Müller, Jean M.: »Einleitung: Phänomenologie der Emotionen«, in: Philosophie der Gefühle, Döring, Sabine [Hrsg.], Frankfurt am Main: Suhrkamp 2009, S. 363.

211 Vgl. Heckmann, Heinz-Dieter: »Qualia-basierte Argumente gegen den Materialismus«, in: Bewusstsein und Repräsentation, Esken, Frank / Heckmann, Heinz-Dieter [Hrsg.], Paderborn: Mentis 1999, S. 331.

212 Chalmers, David J.: The conscious mind. In search of a fundamental theory, New York: Oxford University Press 1996, S. 10. 
sind, neben den üblichen Standardbeispielen (Schmerz, monosensuale Erlebnisse) auch Vorstellungen, Gedanken, Selbstbewusstsein und Emotionen auf. Obwohl letztere weniger spezifisch als Sinneseindrücke seien, durchdringen und »färben « (»pervade and color «) ${ }^{213}$ Emotionen auf ihre je eigene charakteristische Weise unser gesamtes phänomenales Erleben, solange sie gegenwärtig sind:

Emotions often have distinctive experiences associated with them. The sparkle of a happy mood, the weariness of a deep depression, the red-hot glow of a rush of anger, the melancholy of regret: all of these can affect conscious experience profoundly [...]. These emotions pervade and color all of our conscious experiences while they last. ${ }^{214}$

Dementsprechend konstatiert auch Goldie, dass jede Emotion eine charakteristische qualitative Natur (»characteristic qualitative nature $)^{215}$ habe. Pugmire spricht von Gefühlen als »subjektiven Qualitäten« (»subjective qualities«) bzw. »Erlebnisqualitäten« (»experiential quality«), die sich, als phänomenale Kategorie sui generis, nicht in Urteilen bzw. propositionalen Gehalten erschöpfen: »One can be in a certain state of feeling without thinking of oneself as being in it or reflecting on what it is like. « ${ }^{216}$ Auch de Sousa spricht von einer »Emergenz der besonderen Qualia von Gefühlen«.217

Nach Stocker beinhalten nicht alle Emotionen zwingend somatische Empfindungen. ${ }^{218}$ Dennoch geht er davon aus, dass wir, wenn wir bspw. Wut erleben, nicht nur bestimmte evaluative Gedanken und Wünsche haben, sondern auch einen damit verbundenen »Schmerz« empfinden müssen: $» I$ must be pained by the thought of a conspicuous slight $[\ldots] \ll{ }^{219}$ Diesbezüglich stimmt Stocker also mit James überein, denn das bedeutet, dass es möglich ist, dass wir Bewertungen oder Wünsche über eine Situation haben können, die zwar identisch sind mit Bewertungen von Situationen, die uns emotional aufwühlen, aber dennoch keine mit dieser Situation verbundene Emotion

\footnotetext{
213 Ebd.

214 Ebd. [Hervorhebung E. E.].

215 Goldie, Peter: The emotions. A philosophical exploration, New York: Oxford University Press 2002, S. 19.

216 Vgl. Pugmire, David: Rediscovering emotion, Edinburgh: Edinburgh University Press 1998, S. 9o. Das heisst für Pugmire jedoch nicht, dass Gefühle unaussprechlich sind. Vgl. ebd.

217 Sousa, Ronald de / [Übers.: Pape, Helmut]: Die Rationalität des Gefühls, Frankfurt am Main: Suhrkamp [zuerst 1987, engl.]/2009, S. 71.

218 Vgl. Stocker, Michael / Hegeman, Elizabeth: Valuing emotions, Cambridge, Massachusetts: Cambridge University Press 2001, S. 25 und 26.

219 A.a.O., S. 25 .
} 
erleben. Demgemäss ist eine reine Situationsbewertung an sich, nur ein nüchternes Urteil ohne emotionales Erleben:»[...] without feelings, there is no outrage or even anger, but only some >cold-blooded and dispassionate judicial sentence confined entirely to the intellectual realm $[\ldots]<{ }^{220}$ Mit Vogelsang können wir das Fehlen dieser emotionalen Aspekte des Erlebens, bei blossen, nüchternen Urteilen damit begründen, dass sich Emotionen in einer ganz bestimmten Weise anfühlen, ${ }^{221}$ und deshalb auch gegenüber semantischen Gehalten eine »überschiessende Qualität «222 besitzen.

Für Lanz lassen sich die verschiedenen Typen qualitativen Erlebens auch mit Wahrnehmungsverben und im Zusammenhang mit bewusstem Erleben definieren. Visuelles Bewusstsein ist demgemäss ein bestimmtes Sinnesbewusstsein, welches ein Erfahrungssubjekt hat, wenn es etwas Farbiges wahrnimmt, auditives Bewusstsein ist das Sinnesbewusstsein, das wir haben, wenn wir einen Ton hören etc. In Analogie zu den üblicherweise aufgeführten Kategorien von Sinnesbewusstsein kann das Erleben von Emotionen ebenfalls als bewusste Wahrnehmung gesehen werden: »Bewusstsein von Gefühlen, Emotionen, Affekten, Stimmungen ist das Bewusstsein, das man hat, wenn man die Stimmung etc. spürt, in der man sich befindet $[\ldots] \ll{ }^{223}$

Der Umstand, dass sich die üblichen Beispiele für phänomenales Erleben fast ausschliesslich auf die gängigsten Arten von Sinneserlebnissen der Exterozeption beziehen, kann nur schon aufgrund des oft vorkommenden Beispiels interozeptiver Schmerzwahrnehmung kein plausibler Grund dafür sein, dass nur exterozeptive Sinneswahrnehmungen als Qualia gelten sollen. Es gibt auch sonst keinen Grund, warum die bewusste Wahrnehmung von somatoviszeralen Zustandsveränderungen (also von interozeptiven Körperwahrnehmungen) nicht in Analogie zu anderen Körper- und Sinneswahrnehmungen, wie Schmerz oder Farbwahrnehmung, einen weiteren Aspekt eines phänomenal adäquaten Qualia-Begriffs darstellen sollen. Schliesslich stellt die Phänomenologie bzw. der qualitative Aspekt (»phenomenology or the qualitative nature « $)^{224}$ erlebter somatoviszeraler Zustandsveränderungen eine zentrale Eigenschaft von emotionalem Erleben dar, die Goldie zufolge

\footnotetext{
220 A.a.O., S. 26.

221 Vgl. Vogelsang, Frank: Offene Wirklichkeit. Ansatz eines phänomenologischen Realismus nach Merleau-Ponty, Freiburg: Alber 2011, S. 217.

222 Ebd.

223 Lanz, Peter: Das phänomenale Bewusstsein. Eine Verteidigung, Frankfurt am Main: Klostermann 1996, S. 41.

224 Goldie, Peter: The emotions. A philosophical exploration, New York: Oxford University Press 2002, S. 5 2.
} 
zusammen mit der Intentionalität einer Emotion auftritt. ${ }^{225}$ Gefühle bzw. das bewusste Erleben von Emotionen ${ }^{226}$ sind wesentlich ${ }^{227}$ durch solche interozeptiven Körperwahrnehmungen geprägt, die auch in typischen, einer Emotion entsprechenden Wahrnehmungsqualität auftreten:

Fear doesn't feel like a disconnected assortment of bodily symptoms. The bodily components are bound together into a distinctive pattern. [...] The phenomenal character of a freezing episode is qualitatively different from the phenomenal character of a fleeing episode. [...] Conscious experiences of emotion also require attention. Ordinarily emotions grab our attention and are consequently conscious. [...] Emotions are perceptions of bodily changes, and when those perceptions are conscious, emotions are feelings. ${ }^{228}$

Emotionen können deshalb auch Prinz zufolge als eine weitere, neben den Sinneswahrnehmungen bestehende Form der Wahrnehmung betrachtet werden: »If emotions become conscious in just the same way that visual episodes become conscious, then we have a further reason for thinking that emotion is a form of perception $\ll^{229}$

Nach Pugmire weisen Emotionen insofern phänomenale Qualitäten auf, als das erlebte Gefühl dadurch bestimmt ist, wie es sich anfühlt: »How a feeling feels is what makes it the feeling it is «. ${ }^{230}$ Nicht zuletzt lässt sich deshalb mit Engelen ganz allgemein feststellen, dass sich $»[\ldots]$ alle bewussten Vorgänge in einer bestimmten Weise anfühlen [... $\ll^{231}$ und mit phänomenalen Eigenschaften einhergehen: »Angst fühlt sich in einer bestimmen Weise wie Angst an, Freude in einer anderen bestimmten Weise wie Freude, Schmerz wie Schmerz etc. «. ${ }^{232}$ Weil es sich auf eine bestimmte Weise anfühlt, Emotionen zu

225 Vgl dazu auch a.a.O., S. 5o und 57.

226 Nach Prinz gibt es auch unbewusste Emotionen: »Unconscious emotions are also possible, so not all emotions are feelings. Some emotions aren't felt«. Prinz, Jesse: »Are Emotions Feelings? «, Journal of Consciousness Studies, [2005] Vol. 12, No. 8-10, S. 23.

227 Das Erleben von Emotionen wird ebenfalls durch die oben erwähnten, mit ihnen korrelierenden kognitiven Bewertungsprozesse und Denkmodi geprägt. Vgl. dazu z.B. Damasio, Antonio R.: Descartes' error. Emotion, reason and the human brain, London: Vintage [zuerst 1994]/20o6, S. 146. Vgl. ebenfalls Damasio, Antonio R.: Looking for Spinoza. Joy, sorrow and the feeling brain, London: Vintage 2004, S. 89.

228 Prinz, Jesse: »Are Emotions Feelings?«, Journal of Consciousness Studies, [2005] Vol. 12, No. 8-10, S. 23 .

229 Ebd.

230 Pugmire, David: Rediscovering emotion, Edinburgh: Edinburgh University Press 1998, S. 92. Dennoch heisst das für ihn nicht, dass Emotionen ausschliesslich aus phänomenalen Eigenschaften bestehen oder dass sie nicht kommunizierbar sind.

231 Engelen, Eva-Maria: Gefühle, Stuttgart: Reclam 2007, S. 70.

232 Ebd. 
erleben, lässt sich auch von einer "phänomenalen Qualität von Emotionen und Gefühlen «233 oder von »Emotions-Qualia« ${ }^{234}$ sprechen: »[...] an emotional experience is characterized by a certain feeling or felt quality [...]. It is what I have labeled an >emotional quale $\ll{ }^{235}$

Fazit: Im Hinblick auf meine Arbeitsthese, dass phänomenales Erleben auch das Erleben von Emotionen umfasst, können wir abschliessend festhalten, dass der qualitative resp. phänomenale Gehalt eine Gemeinsamkeit emotiver und somatischer Empfindungen darstellt. ${ }^{236}$ Wenn körperlich basierte Wahrnehmungserlebnisse, wie z.B. Schmerz, einen Standardfall von Qualia bilden, muss das mit bewusst erlebten somatoviszeralen Empfindungen eng verbundene Erleben von Emotionen bei einem phänomenal adäquaten Qualia-Begriff mitberücksichtigt werden. Wie oben ausgeführt, umfasst die somatoviszerale Sensibilität die viszerale, somatische und propriozeptive Sensibilität. ${ }^{237}$ Deshalb können die aus diesen Systemen resultierenden psychologisch bzw. emotional relevanten Empfindungen auch als Teil der allgemeinen Sensibilität, die ihrerseits im Zusammenhang mit Emotionen bzw. Gefühlen steht, verstanden werden. Die hier postulierten phänomenalen Gehalte emotiver Erlebnisse werden im Rahmen dieser Arbeit als >emotive Qualia< bzw. als E-Qualia bezeichnet.

\subsubsection{EK-Qualia: Phänomenalität emotiv-kognitiven Erlebens}

Wie oben schon kurz bemerkt, ist Damasio zufolge das bewusste Erleben von Emotionen weder ausschliesslich auf Empfindungen somatoviszeraler Rückmeldungsmuster noch auf Situationsbewertungen beschränkt. ${ }^{238}$ Wenn wir bspw. um Mitternacht nach Hause laufen und plötzlich merken, dass uns jemand folgt, so löst das normalerweise nicht nur einen kühlen Prozess kognitiver Bewertungen und auch nicht nur die blosse körperliche Empfindung von Herzklopfen aus. Die Weise, wie wir auf derart beängstigende Wahrnehmungen reagieren, ist viel komplizierter:

233 A.a.O., S. 121.

234 Diesen Begriff verwendet Calabi, sie verwendet jedoch, aufgrund eines anderen Kontextes, den Singular »emotional quale«. Vgl. Calabi, Clotilde: »Emotional Qualia«, in: Emotions, qualia, and consciousness, Kaszniak, Alfred [Hrsg.], Singapore: World Scientific 2001, S. 77.

235 Ebd.

236 Vgl. Schildknecht, Christiane: »Phänomenales Erleben in Kunst und Literatur«, in: Kunst denken, Burri, Alex [Hrsg.], Paderborn: Mentis 2007, S. 92.

237 Vgl. Birbaumer, Niels / Schmidt, Robert F.: Biologische Psychologie, Berlin: Springer 2003, S. 329 .

238 Vgl. Damasio, Antonio R.: Descartes' error. Emotion, reason and the human brain, London: Vintage [zuerst 1994]/20o6, S. 146 und 224-225. Vgl. ebenfalls Damasio, Antonio R.: Looking for Spinoza. Joy, sorrow and the feeling brain, London: Vintage 2004, S. 89. 
The neural and chemical aspects of the brain's response cause a profound change in the way tissues and whole organ systems operate. [...] the overall biochemical profile of the organism fluctuates rapidly; the skeletal muscles [...] contract; and signals about all these changes are relayed back to the brain $[\ldots] .{ }^{239}$

Und auch diese komplizierten somatischen Prozesse sind nicht alles, was emotionales Erleben kennzeichnet. Emotionsrelevante Eindrücke rufen nicht nur somatische, sondern auch kognitive Prozesse hervor: »Sooner or later, the viscera are made to react to the images you are seeing, and to the images your memory is generating internally, relative to what you see «. ${ }^{240}$ Neben den hier beschriebenen assoziativen, emotionsgeladenen Erinnerungen evoziert bspw. die Situationsbewertung, dass uns jemand folgt wiederum Vorstellungen und somatische Prozesse. ${ }^{241}$ Damasio zufolge stellt also das bewusste Wahrnehmen von viszeralen und propriozeptiven Rückmeldungsmustern, zusammen mit damit einhergehenden Gedanken und einem bestimmten Modus des Gedankenprozesses, die »Essenz «242 emotionalen Erlebens dar:

The essence of sadness or happiness is the combined perception of certain body states with whatever thoughts they are juxtaposed to, complemented by a modification in the style and efficiency of the thought process. ${ }^{243}$

Emotionales Erleben weist, wenn wir die ebenfalls involvierten Situationsbewertungen mitberücksichtigen, folglich drei Elemente auf: ${ }^{244}$ a.) die

239 Damasio, Antonio R.: Descartes' error. Emotion, reason and the human brain, London: Vintage [zuerst 1994]/2006, S. 224.

240 A.a.O., S. 225.

241 So stellt Damasio fest: »The process begins with the conscious, deliberate considerations you entertain about a person or situation. These considerations are expressed as mental images organized in a thought process [...]. At a nonconscious level, networks in the prefrontal cortex automatically and involuntarily respond to signals arising from the processing of the above images«. A.a.O., S. 136. Vgl. dazu auch a.a.O., S. 224-225.

242 A.a.O., S. 146.

243 Ebd. [Hervorhebung von E. E.]. Damasio bemerkt jedoch, dass zu dieser »Essenz« noch weitere Merkmale zu den Emotionen gehören: »I see the essence of emotion as the collection of changes in body state that are induced in myriad organs by nerve cell terminals, under the control of a dedicated brain system, which is responding to the content of thoughts relative to a particular entity or event. [...] But there is more to emotion than its essence. In conclusion, emotion is the combination of a mental evaluative process, simple or complex, with dispositional responses to that process, mostly toward the body proper, resulting in an emotional body state, but also toward the brain itself $[. .$. resulting in additional mental changes«. A.a.O., S. 139 [Hervorhebung im Original].

244 Vgl. dazu Damasio, Antonio R.: Looking for Spinoza. Joy, sorrow and the feeling brain, London: Vintage 2004, S. 89 . 
Wahrnehmung eines bestimmten somatoviszeralen Zustandes; b.) kognitive Bewertungsprozesse, die mit der Emotion korrelieren; ${ }^{245} \mathrm{c}$.) eine Veränderung unseres »Denk-Modus' « (»changes in mode of thinking «). ${ }^{246}$ Die kognitiven Situationsbewertungen interagieren ihrerseits mit somatoviszeralen $\mathrm{Zu}-$ standen, die zudem mit positiven oder negativen Erinnerungen und/oder Zukunftsvorstellungen assoziiert werden. ${ }^{247}$

Die hier beschriebene Interdependenz somatischer, emotionaler und kognitiver Prozesse weist daher darauf hin, dass Gedanken nicht nur Begleiterscheinungen von Emotionen sind, sondern dass sie, wie Deigh feststellt, einen wesentlichen Bestandteil von Emotionen darstellen. ${ }^{248}$ Unsere emotionale Befindlichkeit steht in engem Zusammenhang zu unserem Denken - d.h. zu den jeweils vorherrschenden gedanklichen Inhalten und Bewertungen. Gemäss kognitivistischen Emotionstheorien gilt sogar: »An emotion is a judgment (or a set of judgments) $[\ldots] \ll{ }^{249}$ Spezifische Urteile und Bewertungen, die mit Emotionen oder Gefühlen zusammenhängen, bilden diesen Ansätzen zufolge nämlich die erforderliche Basis, um eine bestimmte Emotion von einer anderen überhaupt unterscheiden zu können. ${ }^{250}$

So sind gemäss Heckmann Hass, Liebe, Neid, Eifersucht, Trauer und Angst in der Regel an epistemische Zustände und kognitive Funktionen gebunden. ${ }^{251}$ Nach Pugmire beinhalten Emotionen deshalb nicht nur Gefühle sondern auch

245 In Damasios Worten:»[...] sort of thoughts that agree, in terms of theme, withe the kind of emotion being felt«. Ebd.

246 Ebd. Mehr über den Zusammenhang zwischen emotionalem Erleben und einem damit einhergehenden mentalen Grundzustand bzw. >Denk-Modus < vgl. weiter unten.

247 Vgl. dazu auch Jung, Matthias / Heilinger, Jan-Christoph [Hrsg.]: Funktionen des Erlebens. Neue Perspektiven des qualitativen Bewusstseins. Band 5: Humanprojekt, Berlin: De Gruyter 2009, S. 19.

248 Vgl. auch Deigh, John: »Cognitivism in the Theory of Emotions«, Ethics, [1994] Vol. 104, No. 4, S. 824 .

249 Solomon, Robert C.: The passions, Notre Dame Indiana: University of Notre Dame Press 1983, S. 185 .

250 Vgl. Hartmann, Martin: Gefühle. Wie die Wissenschaften sie erklären, Frankfurt am Main [u.a.]: Campus 2010, S. 53-54. Allerdings haben kognitivistische Ansätze die Schwäche, dass mit ihnen nicht hinreichend zwischen einem kühlen, nüchternen und einem emotional stark besetzten Urteil unterschieden werden kann, auch wenn beide Urteile auf einer semantischen Ebene identisch sein können: das heisst bspw., dass das Urteil a.) >er hat mich betrogen<, welches bloss eine sachlich-nüchterne Feststellung ist, mit einem strikt kognitivistischen Ansatz nicht vom Urteil b.) >er hat mich betrogen $<$ welches von starken Emotionen begleitet ist, unterschieden werden. Die nüchterne bzw. kaum vorhandene Emotion, die das Urteil a.) begleitet, unterscheidet sich aber von der starken Emotion, die das Urteil b.) begleitet.

251 Vgl. Heckmann, Heinz-Dieter [Hrsg.]: Bewusstsein und Repräsentation, Paderborn: Mentis Verlag 1999, S. 331. 
Gedanken, ${ }^{252}$ er postuliert sogar, dass Emotionen eine »begriffliche Struktur « 253 haben könnten und manche Emotionen auch »normative Aspekte «. ${ }^{254}$ Ellis zufolge rufen Gedanken mit ihnen korrelierende Gefühle hervor, indem wir uns bestimmte gedankliche Sätze innerlich »vorsagen ${ }^{255}$ Je nach der Qualität der Wertung dieser »verinnerlichten Sätze « ${ }^{256}$ empfinden wir gemäss Ellis ein bestimmtes Gefühl. ${ }^{257}$ Emotionen sind nach dieser Konzeption gar eine »stark wertende Art des Denkens «. ${ }^{258}$ Aus all diesen Ausführungen lässt sich ableiten, dass Emotion und Kognition miteinander korrelieren ${ }^{259}$ und dass kognitive Vorgänge äusserst relevant für emotive Zustände sind. Dazu nochmals Damasio:

In effect, normal human behaviour exhibits a continuity of emotions induced by a continuity of thoughts. The contents of those thoughts [...] include objects with which the organism is actually engaged or objects recalled form memory $[\ldots]$. In turn, many of these $>$ streams $\prec$ of thoughts $[\ldots]$ can induce emotions $[\ldots] .{ }^{260}$

252 Vgl. Pugmire, David: Rediscovering emotion, Edinburgh: Edinburgh University Press 1998, S. 104-105.

253 Pugmire, David: »Emotionen und ihre empirische Untersuchung«, in: Philosophie der Gefühle, Döring, Sabine [Hrsg.], Frankfurt am Main: Suhrkamp [zuerst 20o6, engl.]/20o9, S. $35^{8}$.

254 Ebd.

255 Ellis, Albert: Die rational-emotive Therapie. Das innere Selbstgespräch bei seelischen Problemen und seine Veränderung, München: J. Pfeiffer 1977, S. 33.

256 A.a.O., S. 55 .

257 Vgl. dazu auch LeDoux, der von Studien von Richard Lazarus berichtet, die nach LeDoux eindeutig bestätigen, dass die Interpretationen von Situationen stark auf die erlebte Emotion beeinflussen: LeDoux, Joseph E.: Das Netz der Gefühle. Wie Emotionen entstehen, München \& Wien: Hanser 1998, S. 56.

258 Ellis, Albert: Die rational-emotive Therapie. Das innere Selbstgespräch bei seelischen Problemen und seine Veränderung, München: J. Pfeiffer 1977, S. 45. Ellis geht sogar von einer Identität zwischen den meisten Emotion mit Bewertung aus: »Ein Grossteil dessen, [...] was wir als Emotionen bezeichnen, scheinen mithin Urteile bzw. Bewertungen zu sein [...]«. A.a.O., S. 51.

259 Vgl. dazu auch Ramsberger, Gail: »The Human Brain: Understanding the Physical Bases of Intrapersonal Communication«, in: Intrapersonal communication. Different voices, different minds, Vocate, Donna R. [Hrsg.], Hillsdale, New Jersey: Erlbaum 2009, S. 73. Vgl. ebenfalls Sousa, Ronald de / [Übers.: Pape, Helmut]: Die Rationalität des Gefühls, Frankfurt am Main: Suhrkamp [zuerst 1987, engl.]/2009, S. 29.

26o Damasio, Antonio R.: The feeling of what happens. Body, emotion and the making of consciousness, London: Vintage 2000, S. 93. 
Auch wenn sich Emotionen nicht auf propositionale Einstellungen ${ }^{261}$ reduzieren lassen, so kann man emotionsbezogene Gedanken, dass heisst Gedanken, die mit Emotionen korrelieren, als propositionale Einstellungen betrachten, die nicht nur semantisch strukturiert, sondern auch >emotiv gefärbt< sind. ${ }^{262}$ Emotionen sind, aufgrund dieses Bezuges zu Propositionen bzw. propositionalen Einstellungen, aussagenähnliche, strukturierte Gebilde. ${ }^{263}$ So sind nach Engelen Gefühle beim Menschen »fast immer konzeptualisiert oder semantisiert «, ${ }^{264}$ das bedeutet auch, dass die Semantik bzw. das Konzept, welches wir über eine Emotion haben, mit dem »Sich-Anfühlen $\ll^{265}$ der Emotion verbunden wird. Emotionen werden demzufolge einerseits »durch Begriffe geformt «, 266 andererseits werden unsere Begriffe von Emotionen wiederum durch ihre Phänomenologie >gefärbt ${ }^{267}$

Propositionale Einstellungen beschränken sich zudem nicht bloss auf die am meisten diskutierten Fälle wie z.B. »glauben, dass« (»belief«) oder »wünschen, dass« (»desire«), sondern umfassen auch Einstellungen des Hoffens, Befürchtens oder Bereuens. ${ }^{268}$ Infolgedessen stellt sich auch die Frage nach emotiven >Färbungen< bei propositionalen Einstellungen. Wenn Gedanken als kognitive Inhalte, in Form von solchen propositionalen Einstellungen aufgefasst werden, stellt sich die Frage, ob Gedanken emotive Aspekte bzw. Anteile haben. ${ }^{269}$ Denn die kognitiv-emotionalen Gehalte bzw. propositionalen

261 Zu propositionalen Einstellungen vgl. Baker, Lynne R.: »Propositional Attitudes«, in: $A$ companion to the philosophy of mind, Guttenplan, Samuel [Hrsg.], Malden, Massachusetts: Blackwell 2005, S. 488-492. Oder: Schiffer, Stephen: »Thought and Language«, in: $A$ companion to the philosophy of mind, Guttenplan, Samuel [Hrsg.], Malden, Massachusetts: Blackwell 2005, S. 589-591. Vgl. ebenfalls Bäuerle, Rainer: »Proposition«, in: Enzyklopädie Philosophie und Wissenschaftstheorie. Band 3: P-So, Mittelstrass, Jürgen [Hrsg.], Stuttgart: Metzler 2004, S. 364-365.

262 Zum Zusammenhang zwischen propositionalen Gehalten, somatischen Empfindungen, Gedanken und Gefühlen vgl. auch Pugmire, David: Rediscovering emotion, Edinburgh: Edinburgh University Press 1998, S. 94-105.

263 Vgl. dazu auch Demmerling, Christoph / Landweer, Hilge: Philosophie der Gefühle. Von Achtung bis Zorn, Stuttgart: Metzler 2007, S. 6.

264 Engelen, Eva-Maria: Gefühle, Stuttgart: Reclam 2007, S. 33.

265 Ebd.

266 So werden nach Engelen Emotionen, die zwar auch auf angeborenen somatischen Prozessen und Reaktionen beruhen, durch unsere Sozialisation immer auch »semantisiert« und »durch Begriffe geformt«. A.a.O., S. 86.

267 Vgl. a.a.O., S. 33.

268 Vgl. dazu Oppy, Graham: »Propositional attitudes«, 1998, Online: https://www. rep.routledge.com/articles/thematic/propositional-attitudes/v-1 [zuletzt geprüft am 25.09.2018].

269 Vgl. dazu auch Guttenplan, Samuel [Hrsg.]: A companion to the philosophy of mind, Malden, Massachusetts: Blackwell 2005, S. 487. 
Einstellungen wie >ich fürchte, dass dieses Geräusch an der Turbine ein Defekt ist!< oder >ich glaube, dass sie mich tatsächlich liebt<, stehen nicht nur, wie Guttenplan bemerkt, in einem engen Zusammenhang mit Propositionen. ${ }^{270}$ Der Gedanke »ich befürchte stark, dass x passiert« besitzt, neben seinem propositionalen Gehalt, auch einen qualitativen Gehalt, gleichsam eine semotive Färbung<.

Demgemäss hat nach Ellis jeder mentale Zustand sowohl kognitive als auch emotionale Komponenten. ${ }^{271}$ Die dualistische Intuition, ${ }^{272}$ die Gedanken als etwas vollständig Getrenntes von Emotionen behandeln möchte, kritisiert Johnson als einen »dream of pure thought «, 273 der auf der Illusion eines »entkörperten Geistes« (»disembodied mind «) ${ }^{274}$ beruhe. Wir können »mit Gefühl an etwas denken «, 275 in unserem Denken können sich Anteilnahme und Besorgnis breitmachen. ${ }^{276}$ Mithin lässt sich auch ein Einfluss emotiver Zustände auf das Denken für Fälle konstatieren, in denen Emotionen Gedanken hervorrufen, die dieser Emotion qualitativ entsprechen. Pugmire gemäss können Emotionen die Art unserer Reflektion (»one's train of reflection «) ${ }^{277}$ bestimmen und auf unsere Überzeugung einwirken, was Vorurteile und Wunschdenken auch zu implizieren scheinen. Bestimmte Emotionen oder Gefühle können folglich einen kognitiven Prozess erst veranlassen ${ }^{278}$ oder ganz bestimmte Gedanken im Kognitionsfeld einer Person »aufwühlen«. ${ }^{279}$ Je nach Grundstimmung und Emotion denken wir anders, »wählen« ${ }^{280}$ andere

270 Vgl. ebd.

271 Vgl. Ellis, Albert: Die rational-emotive Therapie. Das innere Selbstgespräch bei seelischen Problemen und seine Veränderung, München: J. Pfeiffer 1977, S. 45-46.

272 Vgl. dazu Johnson, Mark: The meaning of the body. Aesthetics of human understanding, Chicago: University of Chicago Press 2007, S. 2-3 und 7.

273 A.a.O., S. 2.

274 Ebd. Diesbezüglich stellt Johnson sogar fest, dass wir mit dem Körper >denken< könnten: »This pervasive illusion of disembodied mind, thought, and meaning is beautifully explored and criticized by the American poet Billy Collins, who unsmasks our dream of pure thought by showing that we can think and imagine only through our bodies«.

275 Goldie, Peter: »Emotionen und Gefühle«, in: Philosophie der Gefühle, Döring, Sabine [Hrsg.], Frankfurt am Main: Suhrkamp [zuerst 2002, engl.]/2009, S. 38 o.

276 Vgl. dazu Stocker, Michael: »Einige Betrachtungen zu intellektuellen Wünschen und Emotionen«, in: Philosophie der Gefühle, Döring, Sabine [Hrsg.], Frankfurt am Main: Suhrkamp [zuerst 2004, engl.]/2009, S. 203.

277 Pugmire, David: Rediscovering emotion, Edinburgh: Edinburgh University Press 1998, S. 7. Vgl. auch a.a.O., S. 35 .

278 Vgl. a.a.O., S. 55 .

279 Vgl. auch Hartmann, Martin: Gefühle. Wie die Wissenschaften sie erklären, Frankfurt am Main: Campus 2010, S. 104.

280 Diesbezüglich konstatiert Ciompi: »Laufend wählen wir aus dem Feld der möglichen kognitiven Wahrnehmungen und Denkverbindungen vorwiegend diejenigen aus, die zu 
»Denkverbindungen $\ll^{281}$ aus, was unsere Wahrnehmung und Kognition in affektspezifischer Weise »färbt « . $^{282}$

Das, was wir metaphorisch unsere >geistige Verfassung « nennen, ist eng mit emotiv >gefärbtem< Denken verknüpft und wird von Ciompi als »Affektlogik«283 beschrieben, die sich als »Freude- und Lustlogik«, ${ }^{284} \gg$ Trauerlogik $\ll^{285}$ oder »Wutlogik«286 manifestieren kann. ${ }^{287}$ So kann nach Schumann »[...] ein Gefühl nicht nur ein Wohlbefinden, sondern auch ein `Wohldenken< repräsentieren «. 288 Wenn Personen dagegen wütend sind, besonders wenn sie in dieser Wut gefangen sind, denken, fühlen und handeln sie Stocker gemäss zumeist in »wütenden Arten und Weisen «; ${ }^{289}$ ihre Gedanken sind dann gewissermassen $>$ wutgefärbte Gedanken. Emotionen sind deshalb, mit Pugmire anders ausgedrückt, »ways we have of minding things «. ${ }^{290}$ Dementsprechend findet sich bei Pugmire die ungewöhnliche Konzeption von so genannten »emotive thoughts«:»[...] thoughts lie at the heart of emotional arousal. In other words, perhaps emotional feelings themselves are thoughts, emotive thoughts $[\ldots] \ll{ }^{291}$ Einen anderen Hinweis auf emotive Aspekte von Gedanken findet man bei den von Pugmire an anderer Stelle erwähnten »embodied appraisals «. ${ }^{292}$

Eine analoge Thematisierung emotiver Aspekte des Denkens findet sich darüber hinaus in Stockers Konzeption der so genannten »emotional thoughts « ${ }^{293}$ Stocker vergleicht diese mit einem emotionalen Fokus, ${ }^{294}$ durch

unserer Grundstimmung passen«. Ciompi, Luc: Die emotionalen Grundlagen des Denkens. Entwurf einer fraktalen Affektlogik, Göttingen: Vandenhoeck \& Ruprecht 1997, S. 96.

281 Ebd.

282 Ebd.

283 A.a.O., S. 45 .

284 A.a.O., S. 192.

285 A.a.O., S. 187-19o.

286 A.a.O., S. 183.

287 Vgl. a.a.O., S. $183^{-192 .}$

288 Schumann, Nadine: Gefühl und Rationalität. Eine philosophische Untersuchung zur Theorie Antonio Damasios, Marburg: Tectum 2010, S. 33.

289 Stocker, Michael: »Einige Betrachtungen zu intellektuellen Wünschen und Emotionen«, in: Philosophie der Gefühle, Döring, Sabine [Hrsg.], Frankfurt am Main: Suhrkamp [zuerst 2004, engl.]/2009, S. 214.

290 Pugmire, David: Rediscovering emotion, Edinburgh: Edinburgh University Press 1998, S. 4 [Hervorhebung im Original].

291 A.a.O., S. 63 [Hervorhebung von E. E.]. Vgl. dazu auch a.a.O., S. 29 und $30-32$.

292 Pugmire, David: »Emotionen und ihre empirische Untersuchung «, in: Philosophie der Gefühle, Döring, Sabine [Hrsg.], Frankfurt am Main: Suhrkamp [zuerst 20o6, engl.]/20o9, S. 332. Vgl. ebenfalls a.a.O., S. 346.

293 Stocker, Michael: Emotional Thoughts«, American Philosophical Quarterly, [1987] Vol. 24, No. 1, S. 6o.

294 Vgl. a.a.O., S. 62. 
den ein »Gedanke sernster genommen « wird als ein anderer, und grenzt den damit verbundenen »emotionalen Ernst« (»emotional seriousness«) $)^{295}$ eines Gedankens von der >Ernsthaftigkeit< einer Überzeugung (»evidential seriousness « $)^{296} \mathrm{ab}$. So kann ein »emotionaler Gedanke«, 297 im Sinne Stockers, von uns >ernster genommen werden als eine von uns gehegte, evidente Überzeugung - selbst wenn sie diesem emotionalen Gedanken widerspricht:

When afraid I focus on the possibility of a crash [...]. Further, when afraid I may focus my attention, straining every mental nerve, on the safety [...]. But even though [...] I focus on, hold fast to, that reassuring evidential statistical belief, what seizes my emotional attention and holds sway emotionally is the thought that there might be a crash. ${ }^{298}$

Auch nach Damasio treten während emotionalem Erleben und damit einhergehenden körperlichen Veränderungen vorwiegend Gedanken auf, die mit dieser Emotion korrelieren. ${ }^{299}$ Dabei bilden die erlebten, somatoviszeralen Zustände des Körpers sogar eine Basisreferenz für die kognitiven Bewertungsprozesse. ${ }^{300}$ Auch der Modus des Denkens bzw. dessen Effizienz korreliert mit einem bei einer Emotion gefühlten somatoviszeralen Zustand: »In general, because both the signal of the body state $[. .$.$] and the style and efficiency of$ cognition were triggered from the same system, they tend to be concordant «. 301 Die Veränderungen des Modus' kognitiver Prozesse werden wiederum durch spezifische Muster chemischer Rückmeldungen verursacht: »The substrate of a feeling is completed by the changes in cognitive processes that are simultaneously induced by neurochemical substances «. ${ }^{302}$ Die mit dem Erleben von Freude oder Traurigkeit einhergehenden Denk-Modi, lassen sich mit Damasio folgendermassen beschreiben:

As an example, the cognitive mode which accompanies a feeling of elation permits the rapid generation of multiple images such that the associative process is richer and associations are made to a larger variety of cues available in the

295 Ebd. Vgl. dazu auch Pugmire, David: Rediscovering emotion, Edinburgh: Edinburgh University Press 1998, S. 22.

296 Stocker, Michael: »Emotional Thoughts«, American Philosophical Quarterly, [1987] Vol. 24, No. 1, S. 62.

297 A.a.O., S. 6o.

298 A.a.O., S. 62 [Hervorhebung im Original].

299 Vgl. Damasio, Antonio R.: Descartes' error. Emotion, reason and the human brain, London: Vintage [zuerst 1994]/2006, S. 145.

300 Vgl. a.a.O., S. 223.

301 A.a.O., S. 147.

302 A.a.O., S. 146. 
images under scrutiny. The images are not attended for long. [...] The extreme of this cognitive mode can be found in manic states. By contrast the cognitive mode which accompanies sadness is characterized by slowness of image evocation, poor association in response to fewer clues, narrower and less efficient inferences, overconcentration on the same image, usually those which maintain the negative emotional response. [...] The extreme of this cognitive mode can be found in depression. ${ }^{303}$

Emotionale Glücksgefühle bewirken nicht nur nach Damasio, ${ }^{304}$ sondern auch nach Ciompi einen schnelleren Modus des Denkens bzw. der Kognition:

So steigen zum Beispiel in euphorischen Zuständen Beweglichkeit und Tempo von kognitiven Prozessen stark and; das Denken wird schliesslich sprunghaft, ausufernd, ideenflüchtig. ${ }^{305}$

Ebenfalls beschreibt Ciompi, dass es etwas Wesentliches der »Freude- und Lustlogik «306 sei, dass ein »Und « möglich werde, weil das »freude- oder lustgeleitete Denken ${ }^{307} \mathrm{im} »[\ldots]$ Erleben einer inneren Öffnung und Weitung $[\ldots] «^{308}$ bestehe. ${ }^{309}$ Analog dazu verwendet auch Pugmire die Wendung von »hedonisch gefärbten Gedanken« (»hedonically toned thought «), ${ }^{310}$ was noch etwas stärker auf den phänomenalen Charakter von emotiven Gedankenformen hinweist. Traurig-depressives emotionales Erleben zeichnet sich demgegenüber dadurch aus, dass sich das Denken auf »[...] wenige rigid immer wieder um die gleichen Themen kreisende Inhalte [... «311 einengt. Auch hier sieht Ciompi, analog zu Damasio, ${ }^{312}$ einen Zusammenhang zwischen der Emotion und einem bestimmten Denk-Modus'. Das Denken engt sich beim Erleben traurig-depressiver Emotionsformen nicht nur ein, sondern es »[...] wird stereotyp und zähflüssig, langsam $[. .$.$] «. ^{313}$ Insgesamt definiert Damasio die

303 A.a.O., S. $163^{-164 .}$

304 Vgl. dazu ebd.

305 Ciompi, Luc: Die emotionalen Grundlagen des Denkens. Entwurf einer fraktalen Affektlogik, Göttingen: Vandenhoeck \& Ruprecht 1997, S. 105.

306 A.a.O., S. 192.

307 Ebd.

308 Ebd. [Hervorhebung E. E.].

309 Vgl. auch a.a.O., S. 193-200.

310 Pugmire, David: Rediscovering emotion, Edinburgh: Edinburgh University Press 1998, S. 75.

311 Ciompi, Luc: Die emotionalen Grundlagen des Denkens. Entwurf einer fraktalen Affektlogik, Göttingen: Vandenhoeck \& Ruprecht 1997, S. 105.

312 Vgl. dazu Damasio, Antonio R.: Descartes' error. Emotion, reason and the human brain, London: Vintage [zuerst 1994]/2006, S. 163-164.

313 Ciompi, Luc: Die emotionalen Grundlagen des Denkens. Entwurf einer fraktalen Affektlogik, Göttingen: Vandenhoeck \& Ruprecht 1997, S. 105. 
Art und Weise, wie emotionales Erleben mit dem Erleben unserer Kognition zusammenhängt, einerseits als »the appearance of thoughts with themes consonant with the emotion $[\ldots] \ll{ }^{314}$ Andererseits stellt er fest, dass wir, zusammen mit einer bestimmten Emotion, auch einen bestimmten $\gg$ Denkmodus « (»mode of thinking «) bzw. einen dafür charakteristischen »mentalen Verarbeitungsstil «(»style of mental processing «) $)^{315}$ erleben.

Wenn nun, wie in Abschnitt 8.2.3 dargelegt, emotive Zustände phänomenales Erleben aufweisen, dann ist es naheliegend, dass auch emotive Formen des Denkenswie»thinking of withfeeling «316 oderaffektspezifische»Färbungen« ${ }^{317}$ unserer Kognition einer bestimmten Klasse phänomenaler Erfahrung zuzurechnen sind. Anders gesagt: Wenn emotiv-phänomenales Erleben unsere Kognition färbt, dann müssen auch »emotional thoughts «, $318 »$ Affektlogik« ${ }^{319}$ und emotive Modi des Denkens phänomenale Aspekte aufweisen. Das Erleben eines emotionsdurchdrungenen Denkmodus' weist phänomenale Qualitäten auf, weil es sich irgendwie >anfühlt,${ }^{320}$ wenn unser Denken bei einer bestimmten Emotion primär mit semotionskonsonanten Themen ${ }^{321}$ beschäftigt ist und die charakteristische Qualität eines Denkmodus' aufweist. ${ }^{322}$

314 Vgl. Damasio, Antonio R.: Looking for Spinoza. Joy, sorrow and the feeling brain, London: Vintage 2004, S. 84.

315 Vgl. ebd.

316 Goldie, Peter: The emotions. A philosophical exploration, New York: Oxford University Press 2002, S. 58. Vgl. auch a.a.O., 59, 71 und 77 .

317 Vgl. dazu auch David Chalmers, der in einer Auflistung von bewussten qualitativen Erfahrungen bei den Emotionen feststellt, dass diese unser gesamtes bewusstes Erleben »färben«. Chalmers, David J:: The conscious mind. In search of a fundamental theory, New York: Oxford University Press 1996, S. 10.

318 Stocker, Michael: »Emotional Thoughts«, American Philosophical Quarterly, [1987] Vol. 24, No. 1, S. 6o.

319 Ciompi, Luc: Die emotionalen Grundlagen des Denkens. Entwurf einer fraktalen Affektlogik, Göttingen: Vandenhoeck \& Ruprecht 1997, S. 45 .

320 Diese ungewohnte Formulierung soll klar machen, dass kognitive Zustände neben intentionalen Gehalten auch phänomenale oder qualitative Gehalte besitzen können. Das heisst, dass es irgendwie ist ein Subjekt mit kognitiven Zuständen zu sein. Vgl. Metzinger, Thomas: Subjekt und Selbstmodell. Die Perspektivität phänomenalen Bewusstseins vor dem Hintergrund einer naturalistischen Theorie mentaler Repräsentation, Paderborn: Mentis 1999, S. 211-212.

321 Vgl. z.B. das Erleben von »emotional thoughts«. Vgl. Stocker, Michael: »Emotional Thoughts«, American Philosophical Quarterly, [1987] Vol. 24, No. 1, S. 6o.

322 Es fühlt sich auch irgendwie an, wenn unser Denken bei einem Glücksgefühl schneller als bei einem Trauergefühl ist. Vgl. dazu Damasio, Antonio R.: Looking for Spinoza. Joy, sorrow and the feeling brain, London: Vintage 2004, S. 84. Vgl. ebenfalls Ciompi, Luc: Die emotionalen Grundlagen des Denkens. Entwurf einer fraktalen Affektlogik, Göttingen: Vandenhoeck \& Ruprecht 1997, S. 105. 
Mit Thomas Nagel ${ }^{323}$ können wir sagen, dass es irgendwie ist, ein Subjekt mit einem bestimmten »Denkmodus ${ }^{324}$ oder einer $»$ Affektlogik ${ }^{325}$ wie z.B. der »Trauerlogik «326 oder der »Wutlogik «327 $\mathrm{zu}$ sein und >trauer- oder wutgefärbte $<$ Gedanken zu haben. ${ }^{328}$ So beschreibt Damasio das kognitive Erleben bei einem Glücksgefühl (»feeling well«) folgendermassen:

[...] You found that your mind was filled with thoughts whose themes created a new wave of pleasurable feeling. [...] You had adopted a mode of thinking in which images had a sharp focus and flowed abundantly and effortlessly. [...] A mode of thinking, a style of mental processing, which increased the speed of image generation $[\ldots] \cdot 329$

Die emotiven Formen des Denkens und die Qualitäten von Denk-Modi bzw. Affektlogiken, die mit unserem emotionalen Erleben einhergehen, zeigen auf, dass Emotionen das »Ganze des Bewusstseins $«^{330}$ ausmachen und nicht einfach nur ein marginales Element seltener, irrationaler Episoden darstellen. Emotionen haben einen privilegierten Status innerhalb des Spektrums unseres phänomenalen Erlebens; nicht nur aufgrund ihrer unauflöslichen Verbindungen zu den körperlichen Erlebnisformen, sondern weil sie (auch aus ontogenetischen Gründen) ${ }^{331}$ unsere Kognition und unser Erleben auf eine fundamentale Weise durchdringen und beeinflussen. So zieht Damasio über die Wichtigkeit emotionalen Erlebens ${ }^{332}$ folgendes Fazit: »And since what

323 Vgl. dazu Nagel, Thomas: »What is it like to be a bat?«, Philosophical Review, [1974] Vol. 83, No. 4.

324 Damasio, Antonio R.: Looking for Spinoza. Joy, sorrow and the feeling brain, London: Vintage 2004, S. 84.

325 Ciompi, Luc: Die emotionalen Grundlagen des Denkens. Entwurf einer fraktalen Affektlogik, Göttingen: Vandenhoeck \& Ruprecht 1997, S. 45.

326 A.a.O., S. 187-19o.

327 A.a.O., S. 183 .

$328 \mathrm{Zu}$ >wutgefärbten< Gedanken bzw. zum Fühlen, Denken und Handeln aus und aufgrund von Wut vgl. auch Stocker, Michael: »Einige Betrachtungen zu intellektuellen Wünschen und Emotionen«, in: Philosophie der Gefühle, Döring, Sabine [Hrsg.], Frankfurt am Main: Suhrkamp [zuerst 2004, engl.]/2009, S. 214.

329 Damasio, Antonio R.: Looking for Spinoza. Joy, sorrow and the feeling brain, London: Vintage 2004, S. 84 [Hervorhebung E.E.].

330 Wils, Jean-Pierre: »Sartre: Emotionen als Urteile«, in: Klassische Emotionstheorien. Von Platon bis Wittgenstein, Landweer, Hilge / Renz, Ursula [Hrsg.], Berlin: De Gruyter 2012, S. 666.

331 Vgl. Damasio, Antonio R.: Descartes' error. Emotion, reason and the human brain, London: Vintage [zuerst 1994]/2006, S. 16o.

332 (Wie erwähnt verwendet Damasio in diesem Zusammenhang den Begriff »feelings«. Mit diesem Begriff bezeichnet er das bewusste Erleben von Emotionen.) 
comes first constitutes a frame of reference for what comes after, feelings have a say on how the rest of the brain and cognition go about their business. Their influence is immense ${ }^{3} .33$

Wirklichkeit verwandelt sich, wenn wir emotional gestimmt sind, in eine »Emotions-Wirklichkeit«. ${ }^{334}$ Emotionales Erleben und Stimmungen wirken sich auf unsere Kognition aus. Die ganze Welt kann sich durch sie in einer »Trauerfärbung «,, 35 aber auch im omnipräsenten Glanz einer munteren Melodie zeigen: »[...] selbst die >strahlende Sonne< scheint >trüber< zu scheinen, oder, obwohl vielleicht draussen graues Herbstwetter ist, >hängt uns doch der Himmel voller Geigen « ${ }^{336}$ Emotionen sind daher, mit Wils formuliert, eine $»$ Bewusstseins- und Weltqualität«. ${ }^{337}$ Sie bewirken diese »Einfärbung der Welt«,338 weil sie »eine Art Hermeneutik der Dinge «339 darstellen und deshalb nicht einfach als von der Kognition isolierte Emotionen, sondern als »[...] Zustand des Bewusstseins, als bedeutungsvolle Bezogenheit auf die Welt $[. ..] \ll^{340}$ aufgefasst werden müssen. So können wir mit Wils feststellen, dass das Erleben von Emotionen und emotiven Denkformen »[...] zu den qualitativen Äusserungen des Bewusstseins und der Wirklichkeitserfassung «341 und insofern zu den zentralen phänomenalen Bewusstseinszuständen unseres Daseins gehören.

Angesichts der Ubiquität emotionaler Formen des Erlebens, die sich in einer kontinuierlichen, affektiven Färbung unserer Erfahrung auszeichnen, ${ }^{342}$ dürfen diese bei keiner Konzeption des Mentalen ausgelassen werden. Eine Philosophie des Geistes, die diesem Namen gerecht werden soll, muss deshalb emotives Erleben berücksichtigen: »It does not seem sensible to leave

333 Ebd.

334 Wils, Jean-Pierre: »Sartre: Emotionen als Urteile«, in: Klassische Emotionstheorien. Von Platon bis Wittgenstein, Landweer, Hilge / Renz, Ursula [Hrsg.], Berlin: De Gruyter 2012, S. 666.

335 Landgrebe, Ludwig / [Hrsg.: Novotný, Karel]:Der Begriff des Erlebens. Ein Beitrag zur Kritik unseres Selbstverständnisses und zum Problem der seelischen Ganzheit, Würzburg: Königshausen \& Neumann [zuerst 1932]/2010, S. 75 .

336 Ebd.

337 Wils, Jean-Pierre: »Sartre: Emotionen als Urteile«, in: Klassische Emotionstheorien. Von Platon bis Wittgenstein, Landweer, Hilge / Renz, Ursula [Hrsg.], Berlin: De Gruyter 2012, S. 667 .

338 A.a.O., S. 671 .

339 Ebd.

340 A.a.O., S. 666.

341 A.a.O., S. 666-667.

342 Vgl. dazu auch Slaby, Jan: »James: Von der Physiologie zur Phänomenologie«, in: Klassische Emotionstheorien. Von Platon bis Wittgenstein, Landweer, Hilge / Renz, Ursula [Hrsg.], Berlin: De Gruyter [zuerst 2008]/2012, S. 562-563. 
emotions and feelings out of any overall concept of mind «.343 Vor dem Hintergrund der Interdependenz von emotiven Zuständen und Gedanken und insbesondere wegen des fundamentalen Einflusses von Emotionen auf das Denken, lässt sich aufgrund des oben dargestellten phänomenalen Gehaltes emotiver Denk-Modi bzw. emotionaler Färbungen unserer Kognition aufzeigen, dass emotiv-kognitive Zustände ebenfalls bei einem phänomenal adäquaten Qualia-Begriff mitberücksichtigt werden müssen. Die für emotionales Erleben wichtigen Aspekte, wie »emotive ${ }^{344}$ resp. »emotionale $\ll^{345} \mathrm{Ge}-$ danken, Affektlogiken, ${ }^{346}$ emotiven Modi des Denkens ${ }^{347}$ und emotionalen Färbungen ${ }^{348}$ der Kognition können aufgrund des Desiderates phänomenaler Adäquatheit bzw. der Beachtung des Explanandums des hard problem nicht einfach aussen vorgelassen werden, nur um phänomenales Erleben sisoliert< und vereinfacht thematisieren zu können. ${ }^{349}$

Wenn wir das hard problem angemessen verstehen möchten, dann ist die Voraussetzung dafür ein phänomenal adäquater Qualia-Begriff. Deshalb müssen wir auch die Phänomenalität dieser für Emotionen grundlegenden emotiv-kognitiven Erlebnisformen als Explanandum beachten, selbst wenn dies auf Kosten der Einfachheit eines traditionellen, homogenen und monosensualen Qualia-Verständnisses geschieht. Die in diesem Abschnitt beschriebenen emotiven Aspekte des Denkens und der Kognition werden im Rahmen dieser Arbeit als >emotiv-kognitives Erleben< bzw. als EK-Qualia bezeichnet. Diese Erlebnisformen emotiver Aspekte der Kognition können im

343 Damasio, Antonio R.: Descartes' error. Emotion, reason and the human brain, London: Vintage [zuerst 1994]/2006, S. 158. In diesem Zusammenhang kritisiert Damasio, dass gerade in einschlägigen kognitionswissenschaftlichen Ansätzen Emotionen bzw. Gefühle ausgelassen werden: »[...] respectable scientific accounts of cognition do precisely that, by failing to include emotions and feelings in their treatment of cognitive systems«. Ebd.

344 Pugmire, David: Rediscovering emotion, Edinburgh: Edinburgh University Press 1998, S. 63. Vgl. dazu auch a.a.O., S. 29 und 30-32.

345 Vgl. Stocker, Michael: »Emotional Thoughts«, American Philosophical Quarterly, [1987] Vol. 24, No. 1. Vgl. auch Stocker, Michael / Hegeman, Elizabeth: Valuing emotions, Cambridge, Massachusetts: Cambridge University Press 2001. Vgl. ebenfalls Stocker, Michael: »Einige Betrachtungen zu intellektuellen Wünschen und Emotionen«, in: Philosophie der Gefühle, Döring, Sabine [Hrsg.], Frankfurt am Main: Suhrkamp [zuerst 2004, engl.]/20o9.

346 Ciompi, Luc: Die emotionalen Grundlagen des Denkens. Entwurf einer fraktalen Affektlogik, Göttingen: Vandenhoeck \& Ruprecht 1997, S. 187-19o.

347 Vgl. Damasio, Antonio R.: Looking for Spinoza. Joy, sorrow and the feeling brain, London: Vintage 2004, S. 84.

348 Vgl. dazu z.B. Chalmers, David J.: The conscious mind. In search of a fundamental theory, New York: Oxford University Press 1996, S. 10.

349 Vgl. dazu auch Slaby, Jan: Gefühl und Weltbezug. Die menschliche Affektivität im Kontext einer neo-existentialistischen Konzeption von Personalität, Paderborn: Mentis 2008, S. 316. 


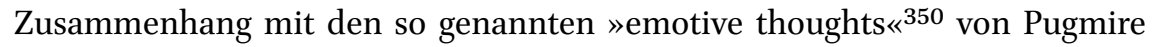
bzw. den »emotional thoughts « ${ }^{351}$ von Stocker auch als emotiv-gedankliche, phänomenale Bewusstseinszustände und somit als >emotive GedankenQualia verstanden werden. Der Einfachheit halber verwende ich jedoch im Hinblick auf diese Kategorie phänomenalen Erlebens ebenfalls die oben erwähnte Begrifflichkeit des >emotiv-kognitiven Erlebensく.

Die Frage, ob Gedanken auch phänomenale Aspekte aufweisen, wenn sie keine emotiven Aspekte besitzen, wird nun zwangsläufig auftauchen. Auf die Frage, ob sich in Abgrenzung zu den EK-Qualia auch nicht-emotive phänomenale Aspekte des Denkens ausweisen lassen, gehe ich im übernächsten Kapitel (Abschnitt 8.2.6) ein.

\subsubsection{I-Qualia: Phänomenale Aspekte des Intentionalen}

Sowohl reduktionistische wie auch nicht-reduktionistische Lösungsversuche des hard problem gehen typischerweise davon aus, dass mentale Zustände grundsätzlich in zwei verschiedenen Varianten auftreten: ${ }^{352}$

1. Als nicht-phänomenale Zustände, in Form begrifflicher Gehalte wie Gedanken und propositionaler Einstellungen

2. Als phänomenale Zustände wie z.B. Sinneswahrnehmungen und Körperempfindungen

Demnach gelten nicht-phänomenale Zustände (1) als Standardfälle intentionaler Gehalte. Phänomenale Zustände (2) gelten demgegenüber gewöhnlich als intrinsische, nicht-relationale ${ }^{353}$ und somit nicht-intentionale ${ }^{354}$ Gehalte:

$35^{\circ}$ Pugmire, David: Rediscovering emotion, Edinburgh: Edinburgh University Press 1998, S. 63.

351 Stocker, Michael: »Emotional Thoughts«, American Philosophical Quarterly, [1987] Vol. 24, No. 1, S. 6o.

$35^{2}$ Vgl. Lycan, William: »Representational Theories of Consciousness«, in: The Stanford Encyclopedia of Philosophy, 2008, Online: http://plato.stanford.edu/archives/fall20o8/ entries/consciousness-representational/ [zuletzt geprüft am 28.o9.2018]. Vgl. auch Pitt, David: »Mental Representation «, in: The Stanford Encyclopedia of Philosophy, 2008, Online: http://plato.stanford.edu/archives/fall20o8/entries/mental-representation [zuletzt geprüft am 25.09.2018].

353 Vgl. z.B. Metzinger, Thomas: Subjekt und Selbstmodell. Die Perspektivität phänomenalen Bewusstseins vor dem Hintergrund einer naturalistischen Theorie mentaler Repräsentation, Paderborn: Mentis 1999, S. 39.

354 Vgl. z.B. Frank, Manfred: »Gibt es eine >innere Wahrnehmung«?«, in: Den eigenen Geist kennen. Selbstwissen, privilegierter Zugang und Autorität der ersten Person, Newen, Albert / Vosgerau, Gottfried [Hrsg.], Paderborn: Mentis 2005, S. 51. Oder: Staudacher, Alexander: Phänomenales Bewusstsein als Problem für den Materialismus, Berlin: De Gruyter 2002. Oder: Willaschek, Marcus: »Wahrnehmung VII (Analytische Philosophie)«, in: Historisches Wörterbuch der Philosophie. Band 12: W-Z, Ritter, Joachim / Kranz, Margarita [Hrsg.], Darmstadt: Wissenschaftliche Buchgesellschaft 1971-2007, 
$»[\ldots]$ nonintentional properties that determine the phenomenal character of experiences are exactly what we have defined as qualia«. ${ }^{355}$ Phänomenales Bewusstsein ist nach dieser Ansicht ein Bewusstseinszustand, der nicht auf einen Gegenstand gerichtet ist. ${ }^{356}$

Entgegen diesem Verständnis gelten für repräsentationalistische Theorien des Geistes Qualia als identisch mit einem mentalen Gehalt, der eine Wahrnehmung repräsentiert. Dieser Gehalt wird als intentionaler Gehalt gesehen, weil er das Objekt einer Wahrnehmung repräsentiert. ${ }^{357}$ Das Vorgehen, Qualia auf intentionale Gehalte zu reduzieren, wird also damit begründet, dass der phänomenale Gehalt bestimmte Eigenschaften des Wahrgenommenen repräsentiere und deshalb eigentlich ein intentionaler Gehalt sei. ${ }^{358}$ Bei Repräsentationalisten gibt es demzufolge zwei Ebenen von intentionalen Gehalten, nämlich eine kognitive Ebene der Intentionalität begrifflicher Gehalte

S. 231. Oder: Block, Ned J.: »Inverted Earth«, in: Philosophy of mind. Critical concepts in philosophy. Volume 4: Consciousness, Crawford, Sean [Hrsg.], London: Routledge \& Kegan Paul [zuerst 199o]/2011, S. 122 und 129. Oder: Metzinger, Thomas: Subjekt und Selbstmodell. Die Perspektivität phänomenalen Bewusstseins vor dem Hintergrund einer naturalistischen Theorie mentaler Repräsentation, Paderborn: Mentis 1999, S. 211.

355 Langsam, Harold L.: »Experiences, Thoughts, and Qualia«, Philosophical Studies, [2000] Vol. 99, No. 3, S. 286.

356 So werden bspw. körperliche Empfindungen und Stimmungen als phänomenale, nicht-intentionale Zustände betrachtet, weil sie angeblich nichts (bzw. keine Objekte) repräsentieren. Vgl. Lycan, William: »Representational Theories of Consciousness«, in: The Stanford Encyclopedia of Philosophy, 2008, Online: http://plato.stanford.edu/archives/ fall2008/entries/consciousness-representational/ [zuletzt geprüft am 28.09.2018], Abschnitt 4.4.1. Lycan wendet sich aber gegen diese Auffassung, weil es im Rahmen des Repräsentationalismus bedeuten würde, dass phänomenale Zustände, die nicht mit Wahrnehmungserlebnissen zu tun haben, von repräsentationalistischen Theorien nicht erfasst würden bzw. vollständig unerklärt blieben: »The representationalist has several options here. First, $\mathrm{s} /$ he could restrict the thesis to perceptual experiences, or to states that are admitted to be intentional. But that would be ad hoc, and would leave the phenomenal character of the excluded mental states entirely unexplained «. Ebd.

357 Vgl. dazu Tye, Michael: »Representationalist Theories of Consciousness«, in: The Oxford Handbook of Philosophy of Mind, McLaughlin, Brian P. / Beckermann, Ansgar / Walter, Sven [Hrsg.], Oxford: Oxford University Press 20o9, S. 262. Vgl. auch Pitt, David: »Mental Representation«, in: The Stanford Encyclopedia of Philosophy, 2008, Online: http://plato. stanford.edu/archives/fall20o8/entries/mental-representation [zuletzt geprüft am 25.09.2018], Abschnitt 3.o.

358 Nicht-reduktionistische Repräsentationalisten wie Block, Loar, Chalmers und Peacocke gehen zwar ebenfalls davon aus, dass Qualia (funktional) repräsentiert sein können; deren irreduzible phänomenale Eigenschaften bestimmen jedoch den intentionalen Gehalt einer Repräsentation. Vgl. a.a.O., Abschnitt 4.o. 
wie Gedanken und propositionale Einstellungen und eine sensorische Ebene der nicht-begrifflichen Intentionalität eines Wahrnehmungsgehalts. ${ }^{359}$

Durch das Zurückführen eines Wahrnehmungsgehalts auf den intentionalen Gehalt einer Repräsentation ${ }^{360}$ wird dann versucht, Phänomenales mit Intentionalem zu identifizieren; ${ }^{361}$ denn Intentionalität gilt bei reduktionistischen Varianten repräsentationalistischer Theorien (Tye, Dretske, Lycan) der Phänomenalität gegenüber als leichter naturalisierbar. ${ }^{362}$ Dem Identifikationsversuch von Qualia mit intentionalen Gehalten, liegt folglich die Motivation zugrunde, naturalistische Erklärungsmodelle des Intentionalen auf das Problem phänomenaler Zustände anzuwenden und auf diese Weise Qualia zu naturalisieren bzw. sie auf Dritte-Person-Eigenschaften wie Repräsentationen zu reduzieren:

The idea is to treat intentionality >objectively<, to treat it as if the subjective features of consciousness did not really matter to it. For example, many functionalists will concede that functionalism can't `handle< consciousness (this is called the problem of qualia), but they think that this issue doesn't matter to their accounts of belief, desire, etc., since these intentional states have no quale, no special conscious qualities. ${ }^{363}$

Es ist jedoch nicht nur phänomenal inadäquat, Qualia auf Repräsentationen >objektiver Eigenschaften bzw. intentionaler Gehalte zu reduzieren, sondern das noch schwerer wiegende Problem dieses Reduktionsversuches liegt darin, dass intentionale Zustände selbst qualitativ-phänomenale Aspekte aufweisen. ${ }^{364}$ Dem Versuch, Qualia auf intentionale Gehalte, die angeblich keine

359 Vgl. Tye, Michael: »Representationalist Theories of Consciousness«, in: The Oxford Handbook of Philosophy of Mind, McLaughlin, Brian P. / Beckermann, Ansgar / Walter, Sven [Hrsg.], Oxford: Oxford University Press 2009, S. 257 und 259. Vgl. ebenfalls Tye, Michael: Ten problems of consciousness. A representational theory of the phenomenal mind, Cambridge, Massachusetts: MIT Press 1995, S. 180.

36o Tye, Michael: »Qualia«, in: The Stanford Encyclopedia of Philosophy, 2007, Online: http:// plato.stanford.edu/archives/fall2007/entries/qualia/ [zuletzt geprüft am 25.09.2018], Abschnitt 7.o.

361 Vgl. dazu auch Schildknecht, Christiane:»Phänomenales Erleben in Kunst und Literatur«, in: Kunst denken, Burri, Alex [Hrsg.], Paderborn: Mentis 2007, S. 97.

362 Vgl. Lycan, William: »Representational Theories of Consciousness «, in: The Stanford Encyclopedia of Philosophy, 2008, Online: http://plato.stanford.edu/archives/fall20o8/ entries/consciousness-representational/ [zuletzt geprüft am 28.o9.2018], Abschnitt 2.4.

363 Searle, John R.: »Consciousness, Unconsciousness and Intentionality«, Philosophical Issues, [1991] Vol. 1: Consciousness, S. 49.

364 So sind nach Graham intentionale Gehalte vom phänomenalen Charakter eines Bewusstseinszustandes nicht isolierbar und vice versa. Vgl. Graham, George / Horgan, Terence / Tienson, John: »Phenomenology, Intentionality, and the Unity of the Mind«, in: 
phänomenalen Eigenschaften haben sollen, zu reduzieren, liegt Searle zufolge ein Irrtum zugrunde. Intentionale Zustände lassen sich nicht vollständig isoliert von Bewusstsein konzipieren: » [...] they think that $[\ldots]$ belief, desire etc. [...] intentional states have no qualia [...] as if they were completely independent of consciousness «. .365 Intentionalität beschränkt sich weder auf repräsentationale Gehalte noch auf Zustände mit begrifflichen Gehalten wie >Überzeugungen $<$ und $>$ Wünsche $<$.

Folgerichtig zeichnet sich nach Janet Levin in der Forschung auch eine zunehmende Übereinstimmung ab, dass intentionale Zustände ebenfalls qualitativ-phänomenale Aspekte aufweisen. ${ }^{366}$ Sogar Standardfälle von Qualia können einen intentionalen Gehalt haben; ${ }^{367}$ so kann sich eine Schmerzempfindung bspw. auf eine Verletzung am Finger beziehen: »[...] a pain is felt as being in a certain part of one's body, as if that part is disordered in a certain way $[\ldots] \ll .368$ Diesbezüglich stellt Lycan fest, dass er und Tye (im Rahmen ihrer repräsentationalistischen Theorien) Brentanos These, dass letztlich alle mentalen Zustände intentional seien, verteidigen würden:

The Oxford Handbook of Philosophy of Mind, McLaughlin, Brian P. / Beckermann, Ansgar / Walter, Sven [Hrsg.], Oxford: Oxford University Press 20o9, S. 523. Auch nach Goldie sind Phänomenalität und Intentionalität unentwirrbar verbunden: »Intentionality and phenomenology are inextricably linked«. Goldie, Peter: »Emotions, feelings and intentionality«, Phenomenology and the Cognitive Sciences, [2002] Vol. 1, No. 3, S. 242.

365 Searle, John R.: »Consciousness, Unconsciousness and Intentionality«, Philosophical Issues, [1991] Vol. 1: Consciousness, S. 49. So stellt Searle fest, dass die Verbindung zwischen Bewusstsein und Intentionalität in Philosophie und Kognitionswissenschaften verloren gegangen sei. Vgl. a.a.O., S. 48. Dem Intentionalitäts-Konzept von Tye fehlt also gewissermassen die geistige Bezugnahme. Vgl. Engelen, Eva-Maria: »Zur Bedeutung von Sprache, Intentionalität und Erleben für das Verständnis von Emotionen«, in: Funktionen des Erlebens. Neue Perspektiven des qualitativen Bewusstseins. Band 5: Humanprojekt, Jung, Matthias / Heilinger, Jan-Christoph [Hrsg.], Berlin: De Gruyter 20o9, S. 39 o.

366 Vgl. Levin, Janet: »Functionalism«, in: The Stanford Encyclopedia of Philosophy, 2013, Online: http://plato.stanford.edu/archives/fall2013/entries/functionalism/ [zuletzt geprüft am 28.09.2018], Abschnitt 4.o. Vgl. dazu auch Pitt, David: »The Phenomenology of Cognition or what is it Like to Think that $\mathrm{P}$ ?«, Philosophy and Phenomenological Research, [2004] Vol. 69, No. 1, S. 1.

367 Vgl. dazu auch Walter, Sven: »Allgemeine Einleitung: Phänomenales Bewusstsein: Unlösbares Mysterium oder seriöses wissenschaftliches Problem?«, in: Qualia. Ausgewählte Beiträge, Heckmann, Heinz-Dieter / Walter, Sven [Hrsg.], Paderborn: Mentis 2001, S. 13-14. Oder: Engelen, Eva-Maria: »Zur Bedeutung von Sprache, Intentionalität und Erleben für das Verständnis von Emotionen«, in: Funktionen des Erlebens. Neue Perspektiven des qualitativen Bewusstseins. Band 5: Humanprojekt, Jung, Matthias / Heilinger, Jan-Christoph [Hrsg.], Berlin: De Gruyter 2009, S. 387 und 394.

368 Lycan, William: »Representational Theories of Consciousness«, in: The Stanford Encyclopedia of Philosophy, 2008, Online: http://plato.stanford.edu/archives/fall2008/ entries/consciousness-representational/ [zuletzt geprüft am 28.o9.2018], Abschnitt 4.4.1. 
[...] representationalists such as Lycan $(1996,2001)$ and Tye $(1995,2003 \mathrm{~b})$ have, in some detail, defended Brentano's thesis that in fact every mental state is intentional, including bodily sensations and moods. It is easy enough to argue that pains and tickles and even orgasms have some representational features $[\ldots] .^{369}$

Goldie wiederum spricht von einem »Wahrnehmungsgehalt körperlicher Empfindungen « (»perceptual account of bodily feelings «), ${ }^{370}$ denn sie beziehen sich auf eine Lokalität. ${ }^{371}$ Körperliche Empfindungen sind insofern auf einen Zustand $^{372}$ oder auf ein Objekt, wie z.B. auf einen Zahn mit Karies, gerichtet, ${ }^{373}$ und können deshalb auch als etwas Intentionales verstanden werden:

When intentionality is thus understood, in terms of directedness towards an object rather than in terms of saboutness<, bodily feelings are unproblematically intentional, being directed towards a part of one's body in a certain location. ${ }^{374}$

Auch die Abneigung, die zusammen mit der Empfindung eines Schmerzes oder eines Ekels gewöhnlich auftritt, kann als Intentionalität verstanden werden; und ohne diese Abneigung ist Schmerzhaftigkeit oder Ekel (im üblichen Sinne) $)^{375}$ kaum vorstellbar. ${ }^{376}$ Ungeachtet der hier aufgezeigten

369 Ebd.

370 Goldie, Peter: »Emotions, feelings and intentionality«, Phenomenology and the Cognitive Sciences, [2002] Vol. 1, No. 3, S. 236.

371 Vgl. dazu auch Perry, John:»Subjectivity«, in: The Oxford Handbook of Philosophy of Mind, McLaughlin, Brian P. / Beckermann, Ansgar / Walter, Sven [Hrsg.], Oxford: Oxford University Press 2009, S. 224 und 23 o.

372 Vgl. dazu Searle, John R.: »What Is an Intentional State?«, Mind (New Series), [1979] Vol. 88, No. 349, S. 75 .

373 Vgl. dazu Goldie, Peter: The emotions. A philosophical exploration, New York: Oxford University Press 2002, S. 37. Schmerz hat Intentionalität, weil er gewöhnlich einen Schaden repräsentiert. Auf diesen Schaden, die Karies, ist Schmerzerleben intentional und gleichsam in einer >widerwillig-abgeneigten Weise gerichtet. Vgl. dazu auch Engelen, EvaMaria: »Zur Bedeutung von Sprache, Intentionalität und Erleben für das Verständnis von Emotionen «, in: Funktionen des Erlebens. Neue Perspektiven des qualitativen Bewusstseins. Band 5: Humanprojekt, Jung, Matthias / Heilinger, Jan-Christoph [Hrsg.], Berlin: De Gruyter 2009, S. 388-389.

374 Goldie, Peter: »Emotions, feelings and intentionality«, Phenomenology and the Cognitive Sciences, [2002] Vol. 1, No. 3, S. 236 [Hervorhebung von E. E.].

375 Davon ausgenommen sind freilich die in Abschnitt 7.1 erwähnten, seltenen, durch Morphineinnahmen hervorgerufenen Erlebnisweisen von \Schmerzen<, die nicht mehr als unangenehm empfunden werden - weshalb sich diesbezüglich auch die Frage stellt, ob der Begriff `Schmerz« auf dieses Phänomen überhaupt noch anwendbar ist. Vgl. dazu Dennett, Daniel C.: »Why You Can't Make a Computer That Feels Pain«, Synthese, [1978] Vol. 38 , No. 3, S. 431.

376 Vgl. dazu auch Staudacher, Alexander: Phänomenales Bewusstsein als Problem für den Materialismus, Berlin: De Gruyter 2002, S. 342. 
Intentionalität körperlicher Empfindungen besteht eine Gemeinsamkeit kognitivistischer Emotionstheorien ${ }^{377}$ darin, dass auch emotives Erleben intrinsisch mit kognitiven Elementen wie Gedanken, Überzeugungen und Urteilen verbunden ist. ${ }^{378}$ Gedanken fungieren demzufolge nicht nur als mögliche Auslöser, sondern als Gehalte von Gefühlen; nach Damasio sogar als deren eigentlicher Gehalt:»[...] I am not inquiring about the cause of the feeling [...] or about what thoughts came into your mind in the wake of the feeling. I really mean the mental contents, the ingredients, the stuff that makes a feeling «.379 Für Pugmire können Gedanken Gehalte von Emotionen sein, selbst wenn uns erstere nicht bewusst sind. ${ }^{380}$ Kognitive Gehalte gewährleisten demnach den Bezug von Emotionen auf die Welt und bestimmen, auf welche Objekte unsere Emotionen gerichtet sind. Emotionstheorien mit kognitivistischem Ansatz zeichnen sich in dieser Hinsicht durch einen Rekurs auf denjenigen Aspekt der Intentionalität aus, anhand dessen Emotionen von >blossen< körperlichen Empfindungen unterschieden werden können. Denn Emotionen sind, im Gegensatz zu vornehmlich ungerichteten, diffusen Stimmungen, ${ }^{381}$ gewöhnlich auf etwas bzw. jemanden gerichtet. 382 Wenn wir Ärger empfinden, so ist damit nicht nur ein bestimmter, angeregter somatischer Zustand verbunden,

377 Vgl. z.B. Helm, Bennett W.: »Felt Evaluations: A Theory of Pleasure and Pain«, American Philosophical Quarterly, [2002] Vol. 39, No. 1. Vgl. ebenfalls Sousa, Ronald de / [Übers.: Pape, Helmut]: Die Rationalität des Gefühls, Frankfurt am Main: Suhrkamp [zuerst 1987, engl.]/2009, S. 24-29.

378 Vgl. auch Slaby, Jan: »James: Von der Physiologie zur Phänomenologie«, in: Klassische Emotionstheorien. Von Platon bis Wittgenstein, Landweer, Hilge / Renz, Ursula [Hrsg.], Berlin: De Gruyter [zuerst 2008]/2012, S. 550 und 556.

379 Damasio, Antonio R.: Looking for Spinoza. Joy, sorrow and the feeling brain, London: Vintage 2004, S. 83 .

380 So stellt Pugmire fest: [...] emotion can be a way of involuntarily and unwittingly laying bare what one really thinks«. Pugmire, David: Rediscovering emotion, Edinburgh: Edinburgh University Press 1998, S. 21.

381 Vgl. dazu Sousa, Ronald de / [Übers.: Pape, Helmut]: Die Rationalität des Gefühls, Frankfurt am Main: Suhrkamp [zuerst 1987, engl.]/20o9, S. 31. Oder: Ciompi, Luc: Die emotionalen Grundlagen des Denkens. Entwurf einer fraktalen Affektlogik, Göttingen: Vandenhoeck \& Ruprecht 1997, S. 63. Oder: Deigh, John: »Cognitivism in the Theory of Emotions«, Ethics, [1994] Vol. 104, No. 4, S. 826. Oder: Searle, John R. / [Übers.: Gavagai, Harvey]: Die Wiederentdeckung des Geistes, München: Artemis-Verlag \& Winkler [zuerst 1992, engl.]/1993, S. 151 und 160-161. Allerdings könnte man mit De Sousa auch behaupten, dass eine Stimmungen in gewissem Sinne auch >alles< als sein intentionales Objekt hat. Vgl. Sousa, Ronald de: »Emotion «, in: A companion to the philosophy of mind, Guttenplan, Samuel [Hrsg.], Malden, Massachusetts: Blackwell 2005, S. 274.

382 Vgl. dazu auch Hartmann, Martin: Gefühle. Wie die Wissenschaften sie erklären, Frankfurt am Main: Campus 2010, S. 54. 
sondern der Ärger hat einen spezifischen, intentionalen Gehalt. ${ }^{383}$ So sind wir bspw. auf eine Person wütend, weil wir glauben, dass sie unfair zu uns war oder wir fürchten uns vor einem bestimmten Tier oder vor der nächsten Volksabstimmung. Auch positive Emotionen weisen diesen Aspekt der Gerichtetheit auf: wir freuen uns auf ein Glas Wein oder darüber, dass eine enge Freundin die Liebe ihres Lebens gefunden hat.

Diese Aufzählung macht klar, dass Emotionen zumeist durch Intentionalität, im Sinne einer Gerichtetheit ${ }^{384}$ auf Objekte, Situationen oder auf Personen charakterisiert sind. So stellt auch Goldie, obschon sein Ansatz kein kognitivistischer ist, fest, dass blossen körperlichen Gefühlen die emotionale Gerichtetheit ${ }^{385}$ auf etwas fehlt: »Kein noch so starkes körperliches Gefühl kann einem allein offenbaren, wovon die Emotion, die man hat, handelt $[\ldots] \ll^{386}$ Auch nach den Ansätzen der Objektbeziehungspsychologie wirken lustbetonte Emotionen wie Freude oder Vergnügen als Attraktoren, die einen positiven Bezug aufweisen und deshalb Bindungen schaffen. ${ }^{387}$ Ciompi gemäss entwickeln sich durch solche positiv-intentionalen Emotionsbezüge aber auch $»$ Bindungen $\ll^{388}$ zu unbelebten Objekten (Spielzeuge, Automobile), Örtlichkeiten (Städte, Räume, Häuser) und sogar zu »geliebten kognitiven Gebilden« «89 mit einer psychischen Dynamik des »Hin-zu« ${ }^{390}$ - das bedeutet für ihn ein »Suchen von grösserer Nähe « ${ }^{391} \mathrm{zu}$ Theorien, Religionen oder Ideologien. Emotionen begründen dementsprechend gerichtete und affektgeleitete Denkund Aufmerksamkeitsformen, die bestimmte Kanalisierungen, Filterungen, Hierarchisierungen, Priorisierungen, Selektierungen, Verknüpfungen und Trennungen von kognitiven Inhalten nach sich ziehen, ${ }^{392}$ was weiter für deren Intentionalität spricht.

383 Vgl. Staudacher, Alexander: Phänomenales Bewusstsein als Problem für den Materialismus, Berlin: De Gruyter 2002, S. 349.

384 Vgl. Goldie, Peter: »Emotions, feelings and intentionality«, Phenomenology and the Cognitive Sciences, [2002] Vol. 1, No. 3, S. 236.

385 Das heisst nach Goldie jedoch nicht, dass reine körperliche Empfindungen insofern, dass sie auch ein auf Objekt, wie bspw. heisse Füsse, gerichtet sein können, nicht als etwas Intentionales gelten können. Vgl. dazu Goldie, Peter: „Emotionen und Gefühle«, in:Philosophie der Gefühle, Döring, Sabine [Hrsg.], Frankfurt am Main: Suhrkamp [zuerst 2002, engl.]/20o9, S. 379 .

386 Ebd. [Hervorhebung von E. E.].

387 Mahler, Margaret S.: Symbiose und Individuation, Stuttgart: Ernst Klett 1972.

388 Ciompi, Luc: Die emotionalen Grundlagen des Denkens. Entwurf einer fraktalen Affektlogik, Göttingen: Vandenhoeck \& Ruprecht 1997, S. 102.

389 Ebd.

390 Ebd. Vgl. auch a.a.O., S. 193 .

391 A.a.O., S. 102.

392 Vgl. a.a.O., S. 96-99. 
Das bedeutet, dass wir je nach Grundstimmung bzw. emotionalem Zustand anders denken und andere »Denkverbindungen « ${ }^{393}$ auswählen, was auch unsere Wahrnehmung in affektspezifischer Weise »färbt «, ${ }^{394}$ das heisst, dass sich unsere Aufmerksamkeit je nach Emotion anders ausrichtet. ${ }^{395}$ Diesbezüglich spricht Ciompi auch von »affektspezifischen Fühl- und Denkbahnen «, 396 was wiederum auf die Gerichtetheit emotiv-kognitiver Zustände hinweist. Auch Stocker konstatiert, dass die Interdependenz von Emotionen und Denken sehr viel mit Aufmerksamkeit und der intentionalen Ausrichtung unsers Fokus zu tun hat:»[...] the significant relations between emotions and thoughts are better understood as forms of attention and focus than as involving evidence and truth « ${ }^{397}$ Auch Damasio bemerkt, dass das Feld assoziierter Gedanken entsprechend einer von uns erlebten emotionalen Qualität limitiert ist:

$[\ldots]$ the mind represents more than well-being. The mind also represents wellthinking. [...] Likewise, feeling sad is not just about a sickness in the body or about a lack of energy to continue. It is often about an inefficient mode of thought stalling around a limited number of ideas of loss. ${ }^{398}$

Analog dazu geht auch Goldie davon aus, dass unsere intentionale Ausrichtung emotionsspezifisch eingeengt werden kann. ${ }^{399}$ Wenn wir Angst haben, so fixiert sich unsere Intentionalität auf diese Furcht, d.h. unser Denken ssucht< gleichsam furchterregenden Sachen oder Umstände und bewirkt so, dass Dinge, die ansonsten recht harmlos sind, in gefährliche transformiert werden. ${ }^{400}$ Wenn uns bspw. während eines Fluges plötzlich eine Furcht vor einem Absturz überwältigt, dann ist unsere Kognition auf gefährliche Signale ausgerichtet, ja sie >sucht< gleichsam das Bedrohliche: »[...] a wave of fear overwhelms you: you grasp the sound as the sound of an engine in trouble [...].

\footnotetext{
393 A.a.O., S. 96.

394 Ebd.

395 So stellt Ciompi fest: »Laufend wählen wir aus dem Feld der möglichen kognitiven Wahrnehmungen und Denkverbindungen vorwiegend diejenigen aus, die zu unserer Grundstimmung passen«. Ebd. Vgl. dazu auch a.a.O., S. 102.

396 A.a.O., S. 182.

397 Stocker, Michael: Emotional Thoughts«, American Philosophical Quarterly, [1987] Vol. 24, No. 1, S. 61.

398 Damasio, Antonio R.: Looking for Spinoza. Joy, sorrow and the feeling brain, London: Vintage 2004, S. 89.

399 Vgl. Goldie, Peter: The emotions. A philosophical exploration, New York: Oxford University Press 2002, S. 15-18.

400 Vgl. a.a.O., S. 58.
} 
You look for signs of worry in the faces of the flight attendants $[. ..] \ll{ }^{401}$ Die emotionsspezifische Gerichtetheit unserer Kognition vergleicht Sartre mit dem Suchen nach einer versteckten Form in einem Bild. ${ }^{402}$ Emotionen >schärfen< also gewissermassen das Bewusstsein und richten es auf Inhalte aus, die mit ihrer Qualität korrelieren. ${ }^{403}$ Diese von Emotionen und Bedeutungen getragene Form unserer $»[\ldots]$ Bezogenheit auf die Welt $[\ldots] \ll^{404}$ weist ebenfalls auf intentionale Aspekte emotiven Erlebens hin, weil Emotionen mit einer mit Semantisierung einhergehenden Intentionalität verbunden sind. Denn emotionales Erleben ist wie sinnliches Erleben durch Sprache und deren Semantisierung geformt. ${ }^{405}$ Trotz dieser Zusammenhänge zwischen der Intentionalität und der Semantisierung von Emotionen, ist emotionales Erleben nicht auf urteilende bzw. begriffliche Formen intentionaler Gehalte und insofern auch nicht auf propositionale Einstellungen reduzierbar. Denn propositionale Einstellungen weisen selbst eine phänomenale Färbung, oder wie Chalmers es bezeichnet, einen »phänomenalen Geschmack « ${ }^{406}$ auf.

Deshalb beschränkt sich auch die mit emotionalem Erleben verbundene Intentionalität nicht auf begriffliche bzw. propositionale Formen der Gerichtetheit. ${ }^{407}$ Die mit der Beschränkung von Intentionalität auf Überzeugungen und Wünsche verbundene Identifikation von Emotionen mit propositionalen Einstellungen (>ich bin überzeugt, dass Q, deshalb fürchte

401 A.a.O., S. 77 [Hervorhebung im Original].

402 Vgl. Sartre, Jean-Paul: Sketch for a theory of the emotions, London: Methuen \& Co 1962, S. 64 .

403 Vgl. dazu auch Engelen, Eva-Maria: Gefühle, Stuttgart: Reclam 2007, S. 44.. Vgl. auch Stocker, Michael / Hegeman, Elizabeth: Valuing emotions, Cambridge, Massachusetts: Cambridge University Press 2001, S. 93 .

404 Wils, Jean-Pierre: »Sartre: Emotionen als Urteile«, in: Klassische Emotionstheorien. Von Platon bis Wittgenstein, Landweer, Hilge / Renz, Ursula [Hrsg.], Berlin: De Gruyter 2012, S. 666.

405 Vgl. dazu auch Engelen, Eva-Maria: »Zur Bedeutung von Sprache, Intentionalität und Erleben für das Verständnis von Emotionen «, in: Funktionen des Erlebens. Neue Perspektiven des qualitativen Bewusstseins. Band 5: Humanprojekt, Jung, Matthias / Heilinger, JanChristoph [Hrsg.], Berlin: De Gruyter 2009, S. 413. Vgl. dazu auch Graham nach dem phänomenale Wahrnehmung auch durch semantische Kompetenz geformt wird: Graham, George / Horgan, Terence / Tienson, John: »Phenomenology, Intentionality, and the Unity of the Mind «, in: The Oxford Handbook of Philosophy of Mind, McLaughlin, Brian P. / Beckermann, Ansgar / Walter, Sven [Hrsg.], Oxford: Oxford University Press 2009, S. 520-521.

406 Chalmers, David J.: The conscious mind. In search of a fundamental theory, New York: Oxford University Press 1996, S. 10.

407 Vgl. dazu auch Evans, Gareth / McDowell, John: The varieties of reference, Oxford: Clarendon Press 1982, S. 143-258. 
mich vor $\mathrm{P}<$ ) durch kognitivistische Emotionstheorien ${ }^{408}$ kommt Goldie zufolge einer »Überintellektualisierung« (»over-intellectualization« $)^{409}$ von Emotionen gleich. Denn die Intentionalität von Emotionen kann bspw. bei Kleinkindern auch vorsprachliche Arten ${ }^{410}$ der Gerichtetheit aufweisen, die nicht von kognitiven Schemata abhängen: »Um sich gefühlsmüssig auf Welt und Mitwelt zu beziehen, muss das Kleinkind nicht wissen, dass es sich auf Welt und Mitwelt bezieht [...] «.411 Emotionales Erleben muss deshalb auch nicht notwendigerweise auf Überzeugungen gründen. Wir können auch grosse oder sogar eine phobische Angst vor einer harmlosen Spinne haben, ohne im Geringsten davon überzeugt zu sein, dass diese Spinne tatsächlich gefährlich ist - dennoch ist unsere Furcht auf den unheimlichen Anblick dieser Spinne gerichtet. ${ }^{412}$

Die kognitivistische These, dass Emotionen auf Überzeugungen oder propositionale Einstellungen reduziert werden können, ignoriert folglich die oft mit körperlichen Empfindungen zusammenhängende und nicht immer von Propositionen abhängige Intentionalität von Emotionen, welche Goldie in seiner Konzeption des »feeling towards « 413 thematisiert. Die Intentionalität von Emotionen, genauer: von körperlich-emotionalen Empfindungen, die

408 Vgl. z.B. Solomon, Robert C.: The passions, Notre Dame, Indiana: University of Notre Dame Press [zuerst 1976]/1983, S. 185. Oder: Deigh, John: »Cognitivism in the Theory of Emotions«, Ethics, [1994] Vol. 104, No. 4, S. 847.

409 Goldie, Peter: The emotions. A philosophical exploration, New York: Oxford University Press 2002, S. 22.

410 Vgl. Hartmann, Martin: Gefühle. Wie die Wissenschaften sie erklären, Frankfurt am Main: Campus 2010, S. 95. Vgl. dazu auch Vogel, Matthias: Medien der Vernunft. Eine Theorie des Geistes und der Rationalität auf Grundlage einer Theorie der Medien, Frankfurt am Main: Suhrkamp 2001, S. 177 .

411 Hartmann, Martin: Gefühle. Wie die Wissenschaften sie erklären, Frankfurt am Main: Campus 2010, S. 95 .

412 Vgl. dazu auch Pugmire, David: Rediscovering emotion, Edinburgh: Edinburgh University Press 1998, S. 3o. Ein anderes Beispiel dafür, dass Emotionen nicht auf Urteilen oder propositionalen Einstellungen basieren, sind Patienten mit einer bilateralen Schädigung des Hippocampus, bei welchen zwar das emotionale Gedächtnis und das Erleben von Emotionen funktioniert, ihr deklaratives Gedächtnis jedoch nicht mehr intakt ist (anterograde Amnesie). Solche Patienten ohne funktionstüchtigen Hippocampus aber mit intakter Amygdala erleben Angst und Schrecken, ohne zu wissen, warum. Allerdings ist eine solche, im Zusammenhang mit einer Funktionsstörung auftretende Angst ohne bewusstes Objekt, analog zu einer diffusen Stimmung, auch ungerichtet bzw. ohne Intentionalität. Vgl. dazu Roth, Gerhard: Das Gehirn und seine Wirklichkeit. Kognitive Neurobiologie und ihre philosophischen Konsequenzen, Frankfurt am Main: Suhrkamp 1994, S. 211.

413 Goldie, Peter: The emotions. A philosophical exploration, New York: Oxford University Press 2002, S. 4. 
mit emotiven Gedanken einhergehen, ist von zentraler Bedeutung in seiner Theorie emotionalen Erlebens. So besteht eine Emotion nach Goldie darin, auf eine ganz bestimmte, emotive Art zu denken, die meistens ${ }^{414}$ mit einer »geliehenen Intentionalität« (»borrowed intentionality«) ${ }^{415}$ der mit dieser Emotion zusammenhängenden körperlicher Empfindung auftritt: »[...] our whole being aches in grief for the one we have lost «. ${ }^{416}$ Mit der Wendung »borrowed intentionality « ${ }^{417}$ will Goldie einerseits darauf hinweisen, dass das mit Kummer verbundene >Stechen in der Brust<, kein »rein körperliches Empfinden ${ }^{418}$ darstellt und dass dieses Stechen in Bezug zu jemandem steht, der oder die »bekümmert « 419 wird. Sowohl das Ganze unseres Geistes als auch des Körpers sind demnach intentional beteiligt am emotionalen Erleben:

[...] our entire mind and body is engaged in the emotional experience, and all the feelings are sunited in consciousness $<$ in being directed towards its object: united sbody and souk, , heart and mindk. For example, sexual desire is felt with the whole being - body and soul - for the one we desire. ${ }^{420}$

Da es nun aber körperliche Empfindungen gibt, die »just bodily«421 seien und überhaupt keine Intentionalität aufweisen, ausser ihrer Gerichtetheit auf den eigenen Körper (bzw. die betroffene Körperstelle), haben diejenigen Körperempfindungen, die auf ein Objekt einer Emotion gerichtet sind, gleichsam eine >geliehene< Intentionalität: »So the bodily feeling is thoroughly infused with the intentionality of the emotion; and, in turn, the feeling towards is infused with a bodily characterization $\ll .422$

Ein derartiges, mit Emotionen in unentwirrbarer Verschränkung stehendes somatisches Erleben, ist Goldie zufolge ein »[...] ineliminable part of the intentionality of emotional experience $\ll .{ }^{423}$ Emotionen bestehen deshalb nicht

414 Goldie schliesst dabei nicht aus, dass es auch Emotionen ohne körperliche Empfindungen (und die mit ihnen verbundene »geliehene Intentionalität«) gibt, vgl. a.a.O., S. 56. Dennoch sind für ihn körperliche Empfindungen ein überaus wichtiger, nicht zu ignorierender Bestandteil des emotionalen Erlebens: »[...] it is glaringly obvious that feelings are, to say the least, relevant to emotional experience and cannot be left out of the picture $[\ldots] \ll$. A.a.O., S. 4.

415 A.a.O., S. 54 .

416 A.a.O., S. 55 [Hervorhebung im Original].

417 A.a.O., S. 54.

418 Ebd.

419 A.a.O., S. 55 .

420 Ebd. [Hervorhebung im Original].

421 A.a.O., S. 56.

422 A.a.O., S. 57 [Hervorhebung E. E.].

423 A.a.O., S. 4. 
lediglich aus (empfindungslosen) propositionalen Einstellungen, sondern auch aus >emotionsgeladenen< Gehalten (»emotionally laden content $[\mathrm{s}] \ll)^{424}$, die zumeist mit körperlichen Empfindungen zusammenhängen. Intentionale Zustände können, im Zusammenhang mit der vorherigen Ausweisung der Phänomenalität emotionalen Erlebens, deshalb ebenfalls körperlich-emotive Qualia aufweisen. Auch im Rahmen von Goldies Konzeption des »feeling towards « ${ }^{425}$ und den damit verbundenen qualitativen Aspekten der Intentionalität von Emotionen muss ein phänomenal adäquater Qualia-Begriff um diesen Bereich somatisch-emotional-intentionaler Erlebniszustände erweitert werden, die ich im Rahmen dieser Arbeit als phänomenale Intentionalität bzw. als I-Qualia bezeichnen werde.

Die in diesem Abschnitt dargelegten Gründe, warum Intentionales selbst phänomenale Aspekte aufweisen kann, lassen folgendes Fazit zu: Qualia lassen sich nicht auf intentionale Zustände reduzieren, die angeblich keinerlei phänomenale Gehalte bzw. Aspekte des Erlebens besitzen, weil sich Formen von Intentionalität ausweisen lassen, die nicht von Phänomenalität getrennt werden können:

[...] there is a type of intentionality that is entirely constituted phenomenally, and it pervades our mental lives. This is phenomenal intentionality. [...] Phenomenal intentional content is individuated phenomenologically. 426

\subsubsection{D-Qualia: Phänomenale Aspekte des Denkens}

In der analytischen Philosophie des Geistes werden Gedanken (auch deren emotive Aspekte) fast nie mit Qualia in Verbindung gebracht, so werden Gedanken beispielsweise in repräsentationalistischen Ansätzen als grundsätzlich andere Kategorie mentaler Zustände im Vergleich zu Qualia betrachtet:

It is a traditional assumption among realists about mental representations that representational states come in two basic varieties [...]. There are those, such as thoughts, which are composed of concepts and have no phenomenal (>what-it'slike $<$ ) features (>qualia<), and those, such as sensations, which have phenomenal features but no conceptual constituents. ${ }^{427}$

\section{Ebd.}

425 A.a.O., S. 57 .

426 Graham, George / Horgan, Terence / Tienson, John: »Phenomenology, Intentionality, and the Unity of the Mind«, in: The Oxford Handbook of Philosophy of Mind, McLaughlin, Brian P. / Beckermann, Ansgar / Walter, Sven [Hrsg.], Oxford: Oxford University Press 2009, S. 53 o [Hervorhebung von E. E.].

427 Pitt, David: »Mental Representation«, in: The Stanford Encyclopedia of Philosophy, 2008, Online: http://plato.stanford.edu/archives/fall2oo8/entries/mental-representation [zuletzt geprüft am 25.09.2018], Abschnitt 3.0 [Hervorhebung von E. E.]. 
Ned Block räumt jedoch an prominenter Stelle - im Oxford Companion to the Philosophy of Mind - ein: »Qualia are experiential properties of sensations, feelings, perceptions and, in my view, thoughts and desires as well «.428 Doch inwiefern können denn nüchterne, >gewöhnlicheく Gedanken, d.h. solche, die keine emotiven Aspekte besitzen, überhaupt mit phänomenalem Erleben und Qualia in Verbindung gebracht werden? Hier lässt sich mit Landgrebe entgegnen, dass wir, sofern wir nicht bewusstlos sind, in jedem Moment unseres Lebens immer irgendetwas erlebt haben - bzw. erleben werden. ${ }^{429}$ Gedanken und bewusstes Erleben müssen mindestens insoweit zusammenhängen, als es unmöglich ist, einen Gedanken zu denken, ohne dass uns dieser bewusst ist. Deshalb kann eine Konzeption >unbewusster Gedanken $^{430}$ nicht dem entsprechen, was wir unter >Denken< verstehen: Denken und das damit zusammenhängende Wissen-um-Gedanken, sind nur möglich, wenn sie sich im >Aufmerksamkeitshorizont $<$ des Bewusstseins befinden und nicht im Dunkeln des Unbewussten ein epistemisch unsichtbares Dasein fristen. ${ }^{431}$ So stellt McGinn die berechtigte Frage: »Could our thoughts have the very content they now have if we were not to be conscious beings at all? «. ${ }^{432}$ Dass ich bewusst bin, ist konstitutiv ${ }^{433}$ für mein Denken »[...] wäre ich es nicht, so wäre dies

428 Block, Ned J.: »Qualia«, in: A companion to the philosophy of mind, Guttenplan, Samuel [Hrsg.], Malden, Massachusetts: Blackwell 2005, S. 514 [Hervorhebung E.E.].

429 Vgl. Landgrebe, Ludwig / [Hrsg.: Novotný, Karel]: Der Begriff des Erlebens. Ein Beitrag zur Kritik unseres Selbstverständnisses und zum Problem der seelischen Ganzheit, Würzburg: Königshausen \& Neumann [zuerst 1932]/2010, S. 5 o.

430 Vgl. dazu auch Searle, John R.: »Consciousness, Unconsciousness and Intentionality«, Philosophical Issues, [1991] Vol. 1: Consciousness, S. 57-58.

431 Vgl. ebenfalls Chalmers, David J.: The conscious mind. In search of a fundamental theory, New York: Oxford University Press 1996, S. 13.

432 McGinn, Colin:»Thought «, in: A companion to the philosophy of mind, Guttenplan, Samuel [Hrsg.], Malden, Massachusetts: Blackwell 2005, S. 587 .

433 Das heisst freilich nicht, wie Metzinger zu Recht bemerkt, dass wir nur selten klar und bewusst unser Denken erleben und dass der allergrösste Teil unseres Gedanken, die in frei assoziierender Weise auftauchen, eher nur halbbewusst ist: »[...] das ständige Geplapper eines automatisch ablaufenden inneren Monologs, das Hintergrundgeräusch aus Erinnerungen, Bewertungen und kleinen Geschichten, welches wie ein Schleier die Wahrnehmung des aktuellen Moments trübt. Nur manchmal haben wir das Erlebnis, beim Denken wirklich innerlich Handelnde zu sein. Meist ist es jedoch so, dass Gedanken einfach vorbeiziehen wie Wolken am Himmel«. Metzinger, Thomas: Der Ego Tunnel. Eine neue Philosophie des Selbst: Von der Hirnforschung zur Bewusstseinsethik, Berlin: Berliner Taschenbuch Verlag [zuerst 2009, engl.]/2011, S. 177. Ein >Denken<, welches nur halbbewusst ist, umschreibt man, erstens jedoch treffender als ein mehr oder weniger >automatisches Assoziieren<. Zweitens heisst das nicht, dass dieses Assoziieren, bei welchem wir uns nicht als »innerlich Handelnde« erleben, nicht trotzdem von Bewusstsein begleitet sein sollte. Drittens bedeutet das auch nicht, dass es nicht irgendwie ist, wenn wir 
Denken niemandes Denken, ging unbemerkt vorüber und wäre somit gar kein Denken [...]«.434 Mit Landgrebe ausgedrückt, ist der Boden, auf dem wir alle Erlebnisse - auch das Erleben von unserem Denken - haben, das »Sein bei Bewusstsein ${ }^{435}$ Aus diesem Grund lassen sich auch nach Lanz nicht nur emotionales Erleben oder die gewöhnlich aufgeführten Kategorien sinnlichphänomenalen Erlebens als Formen von bewusstem Erleben sehen. Analog zur von ihm festgestellten Phänomenalität von emotionalem Erleben ${ }^{436}$ stellt er fest: »Bewusstsein von Gedanken ist das Bewusstsein, das man hat, wenn man bestimmte Gedanken denkt « ${ }^{437}$ Diese Aussage von Lanz ist nicht einfach nur eine Tautologie, sondern sie soll den Zusammenhang zwischen dem bewussten Erleben eines mentalen Zustandes und dessen Phänomenalität klar machen: ein bewusster Gedanke weist, wie ein bewusstes Schmerzerlebnis oder eine bewusste Emotion, seine spezifische Phänomenalität auf.438 Denn wenn es nicht irgendwie wäre, davon überzeugt zu sein, dass 5 eine Primzahl ist, wäre es fragwürdig, ob propositionale Einstellungen überhaupt als bewusste mentale Zustände gelten könnten. ${ }^{439}$ Mit Pitt lässt sich diesbezüglich ein relativ triviales, auf dem modus ponens aufbauendes Argument aufführen, warum Gedanken, wenn sie bewusst sind (wie andere bewusste mentale $\mathrm{Zu}$ stände), Phänomenalität aufweisen:

uns beim Denken als »innerlich Handelnde« erleben; und viertens hat dies nicht zur Folge, dass es nicht irgendwie ist, wenn wir halbbewusst assoziieren und dabei Gedanken »wie Wolken am Himmel« vorbeiziehen.

434 Bermes, Christian: Maurice Merleau-Ponty zur Einführung, Hamburg: Junius 2004, S. 114.

435 Landgrebe, Ludwig / [Hrsg.: Novotný, Karel]: Der Begriff des Erlebens. Ein Beitrag zur Kritik unseres Selbstverständnisses und zum Problem der seelischen Ganzheit, Würzburg: Königshausen \& Neumann [zuerst 1932]/2010, S. 5 o.

436 Zu emotionalem Erleben stellt er fest: »Bewusstsein von Gefühlen, Emotionen, Affekten, Stimmungen ist das Bewusstsein, das man hat, wenn man die Stimmung etc. spürt, in der man sich befindet [...]«. Lanz, Peter: Das phänomenale Bewusstsein. Eine Verteidigung, Frankfurt am Main: Klostermann 1996, S. 41.

437 Ebd. Zur Wahrnehmung von Gedanken vgl. auch Damasio, Antonio R.: Looking for Spinoza. Joy, sorrow and the feeling brain, London: Vintage 2004, S. 86.

438 Vgl. Lanz, Peter: Das phänomenale Bewusstsein. Eine Verteidigung, Frankfurt am Main: Klostermann 1996, S. 41. Vgl. dazu auch Pitt, David: »The Phenomenology of Cognition or what is it Like to Think that P?«, Philosophy and Phenomenological Research, [2004] Vol. 69, No. 1, S. 2.

439 Vgl. Jacob, Pierre: »Intentionality«, in: The Stanford Encyclopedia of Philosophy, 2010, Online: http://plato.stanford.edu/archives/fall2o1o/entries/intentionality/ [zuletzt geprüft am 28.09.2018], Abschnitt 10.o. Zum Erleben propositionaler Gehalte, wie dem >Erleben von Urteilen<, vgl. auch Landgrebe, Ludwig / [Hrsg.: Novotný, Karel]: Der Begriff des Erlebens. Ein Beitrag zur Kritik unseres Selbstverständnisses und zum Problem der seelischen Ganzheit, Würzburg: Königshausen \& Neumann [zuerst 1932]/2010, S. 5 o. 
Prämisse 1: If a mental state is conscious, then it has phenomenal properties.

Prämisse 2: Conscious thoughts are conscious mental states; therefore,

Konklusion: Conscious thoughts have phenomenal properties. ${ }^{440}$

Das Gemeinsame von sinnlich-phänomenalem Erleben und erlebten Gedanken besteht demnach darin, dass beide Formen mentaler Zustände bewusst sein müssen, damit es irgendwie ist, sie zu erleben: ${ }^{441}$

And just as what it is like for a subject to have a perceptual experience is constituted by what it is like for him to have conscious access to certain features of that experience, similarly, what it is like for a subject to have a thought is constituted by what it is like for him to have conscious access to certain features of that thought. ${ }^{442}$

440 Pitt, David: »The Phenomenology of Cognition or what is it Like to Think that P?«, Philosophy and Phenomenological Research, [2004] Vol. 69, No. 1, S. 2.

441 Deshalb wäre es auch unsinnig von >unbewusstem phänomenalen Erleben< oder >unbewussten Qualia< im Zusammenhang mit dem Phänomen der Blindsicht zu sprechen. Damit wird freilich nicht das Phänomen der Blindsicht geleugnet. Bei Phänomen der Blindsicht geben Patienten an, dass sie nichts sehen, bei der Aufforderung zu erraten, wo sich bspw. etwas bewegt, erzielen sie aber immerhin bessere Ergebnisse, als wenn sie nur zufällig raten würden. Jemand mit Blindsicht hat also keine visuellen Erlebnisse, doch bestimmte funktionale Leistungen, die mit Sehen in Verbindung stehen, scheinen noch reduziert vorhanden zu sein. Auch wenn bei der Blindsicht eine funktionale Restleistung, die mit Sehen zu tun hat, zwar noch feststellbar ist, so ist es für einen Patienten mit Blindsicht aber nicht irgendwie zu sehen, sondern er oder sie erlebt sich als Blind und hat insofern auch keine visuellen phänomenalen Erlebnisse. Aus diesem Grund ist es auch im Zusammenhang mit Blindsicht unsinnig, von >unbewusstem phänomenalen Erleben zu sprechen. Vgl. dazu auch Stubenberg für den die Konzeption >unbewusster Qualia absurd ist: Stubenberg, Leopold: Consciousness and qualia, Amsterdam: John Benjamins 1998, S. 71. Meine Verwendungsweisen von phänomenalen Erleben bzw. von Qualia unterscheiden sich diesbezüglich von derjenigen von Ned Block, nach dem es phänomenales Bewusstsein ohne Zugangsbewusstsein gibt. Vgl. dazu Block, Ned J.: »Das neuronale Korrelat des Bewusstseins«, in: Bewusstsein und Repräsentation, Esken, Frank / Heckmann, Heinz-Dieter [Hrsg.], Paderborn: Mentis [zuerst 1996, engl.]/1999, S. 157. Vgl. auch Block, Ned J.: »On a confusion about a function of consciousness «, Behavioral and Brain Sciences, [1995] Vol. 18, No. 2, S. 231-233. Zur Blindsicht vgl. auch Chalmers, David J.: The conscious mind. In search of a fundamental theory, New York: Oxford University Press 1996, S. 226.

442 Langsam, Harold L.: »Experiences, Thoughts, and Qualia«, Philosophical Studies, [200o] Vol. 99, No. 3, S. 272 [Hervorhebung von E. E.]. 
Nach Strawson können Gedanken insofern Qualia haben, als das plötzliche Denken oder Erinnern an etwas einen Erlebnischarakter hat. ${ }^{443}$ Chalmers spricht diesbezüglich von der »qualitativen Empfindung«, die mit dem Auftreten eines Gedankens einhergeht (»the qualitative feel of an occurrent thought «). ${ }^{444}$ Infolgedessen können wir mit Chalmers, ${ }^{445}$ Langsam, ${ }^{446}$ Perry, ${ }^{447}$ Searle, ${ }^{448}$ Jacob, ${ }^{449}$ Graham et al., ${ }^{450}$ Kobes $^{451}$ und Pitt ${ }^{452}$ behaupten, dass es irgendwie ist, Gedanken mit einem ganz bestimmten Gehalt zu haben:

When I think of a lion, for instance, there seems to be a whiff of leonine quality to my phenomenology: what it is like to think of a lion is subtly different from what it is like to think of the Eiffel tower. ${ }^{453}$

443 Strawson, Galen: Mental reality, Cambridge, Massachusetts: MIT Press 1994, S. 196. Vgl. dazu auch Tye, Michael: »Qualia«, in: The Stanford Encyclopedia of Philosophy, 2007, Online: http://plato.stanford.edu/archives/fall2007/entries/qualia/ [zuletzt geprüft am 25.09.2018], Abschnitt 2.0.

444 Chalmers, David J.: The conscious mind. In search of a fundamental theory, New York: Oxford University Press 1996, S. 10.

445 Vgl. a.a.O., S. 6 und 10.

446 Langsam, Harold L.: »Experiences, Thoughts, and Qualia«, Philosophical Studies, [200o] Vol. 99, No. 3, S. 272.

447 »There is something it is like to think a thought«. Perry, John: »Subjectivity«, in: The Oxford Handbook of Philosophy of Mind, McLaughlin, Brian P. / Beckermann, Ansgar / Walter, Sven [Hrsg.], Oxford: Oxford University Press 2009, S. 224.

$448 \mathrm{Vgl}$ dazu auch Searle nach dem es für uns irgendwie ist, wenn wir etwas bewusst denken: »Is there something it is like, or feels like, just to sit and consciously think that $2+3=5$ ? [...] There is indeed something that it is like, or feels like, to think these things [...] Searle, John R.: The mystery of consciousness, New York: New York Review Books 1997, S. 201.

449 Jacob, Pierre: »Intentionality«, in: The Stanford Encyclopedia of Philosophy, 2010, Online: http://plato.stanford.edu/archives/fall2010/entries/intentionality/ [zuletzt geprüft am 28.09.2018], Abschnitt 10.0.

450 Zitat Graham et al.: »There is something it is like to see a vivid red [...] and to entertain the thought that it is sunny in Arizona. Each of these mental states has a conscious character, with phenomenal or phenomenological properties [...] «. Graham, George / Horgan, Terence / Tienson, John: »Phenomenology, Intentionality, and the Unity of the Mind «, in: The Oxford Handbook of Philosophy of Mind, McLaughlin, Brian P. / Beckermann, Ansgar / Walter, Sven [Hrsg.], Oxford: Oxford University Press 20o9, S. 513. Vgl. auch a.a.O., S. 516.

$45^{1}$ Zitat Kobes: »For any A-conscious [access-conscious] thought token (e. g., that there are infinitely many twin primes, that God is eternal, that there was once an RNA world), there is something it is like to think it, even if there is no particular image or sensory quality or feeling tone intrinsic to the thought «. Kobes, Bernard W.: »Access and what it is like«, Behavioral and Brain Sciences, [1995] Vol. 18, No. 2, S. 26o [Ergänzung in Klammern von E.E.].

$45^{2}$ Vgl. Pitt, David: »The Phenomenology of Cognition or what is it Like to Think that P? «, Philosophy and Phenomenological Research, [2004] Vol. 69, No. 1, S. 2.

453 Chalmers, David J.: The conscious mind. In search of a fundamental theory, New York: Oxford University Press 1996, S. 10 [Hervorhebung von E. E.]. An anderer Stelle drückt 
Denken weist deshalb, neben seinen propositionalen Gehalten, auch qualitative Aspekte auf, weil ein Unterschied im repräsentationalen Gehalt eine phänomenale Differenz nach sich ziehen kann. Ned Block stellt diesbezüglich fest: »I would also add, that thoughts, wants and emotions often have characteristic conscious aspects, and that a difference in representational content can make a phenomenal difference $\ll .454$ So hat beispielsweise hat der Gedankengehalt >meine Partnerin ist eine wunderbare Rose< eine andere phänomenale Qualität als der Gehalt >mein Leben gleicht zunehmend einem Jauchebad ${ }^{455}$ Treffend veranschaulicht dies Graham:»[...] an Arizona thought $[\ldots]$ is distinctively different than what is it like to think about, say, Alaska «. ${ }^{456}$ Im Hinblick darauf, dass es irgendwie ist, Gedanken mit einem bestimmten Gehalt zu haben, spricht Pitt von der »phenomenology of cognition «. $457 \mathrm{Ihm}$ zufolge konstituiert Phänomenales sogar den Gehalt eines Gedankens:

In addition to arguing that there is something it is like to think a conscious thought, I shall also argue that what it is like to think a conscious thought is distinct from what it is like to be in any other kind of conscious mental state, that what it is like to think the conscious thought that $p$ is distinct from what it is like to think any other conscious thought, and that the phenomenology of a conscious thought is constitutive of its content. ${ }^{458}$

Im Zusammenhang mit den, in diesem Zitat von Pitt erwähnten phänomenalen Eigenschaften sui generis der Kognition lässt sich dennoch eine Parallele zwischen Gedanken- und Sinnes-Qualia feststellen. Gedanken haben ihre spezifische Phänomenalität, weil sie - analog zu exterozeptiven phänomenalen Wahrnehmungserlebnissen - gleichsam durch eine >kognitive Wahrnehmung bewusst werden:

dies Chalmers auch so aus, dass es irgendwie ist, jemand zu sein, die oder der kognitive Fähigkeiten hat »I am talking about the subjective quality of experience: what it is like to be a cognitive agent«. A.a.O., S. 6 .

454 Block, Ned J.: »Consciousness «, in: A companion to the philosophy of mind, Guttenplan, Samuel [Hrsg.], Malden, Massachusetts: Blackwell 2005, S. 211.

455 Vgl. dazu ebenfalls Walter, Sven: AAllgemeine Einleitung: Phänomenales Bewusstsein: Unlösbares Mysterium oder seriöses wissenschaftliches Problem?«, in: Qualia. Ausgewählte Beiträge, Heckmann, Heinz-Dieter / Walter, Sven [Hrsg.], Paderborn: Mentis 2001, S. 13.

456 Graham, George / Horgan, Terence / Tienson, John: »Phenomenology, Intentionality, and the Unity of the Mind «, in: The Oxford Handbook of Philosophy of Mind, McLaughlin, Brian P. / Beckermann, Ansgar / Walter, Sven [Hrsg.], Oxford: Oxford University Press 2009, S. 517 .

457 Vgl. Pitt, David: »The Phenomenology of Cognition or what is it Like to Think that P? Philosophy and Phenomenological Research, [2004] Vol. 69, No. 1, S. 2.

458 Vgl. ebd. [Hervorhebung im Original]. 
$[\ldots]$ just as I can close my eyes and have an occurrent, conscious thought that there is a red flag in front of me [...] I can open my eyes and have a visual experience $[. .$.$] Conscious thoughts are conscious in the same sense that percep-$ tual experiences are conscious: they have phenomenal character. Just as there is something it is like for a subject to have a perceptual experience, so, too, there is something it is like for a subject to have a (conscious) thought. ${ }^{459}$

Die These, dass Gedanken, analog zum herkömmlichen Verständnis sinnlichexternem phänomenalen Erleben, gewissermassen >innerlich wahrgenommen< werden können, stimmt mit der Konzeption von HOP-Theorien ${ }^{460}$ insoweit überein, als bewusstes Erleben und Introspektion als zutiefst verwoben betrachtet werden. ${ }^{461}$ Von Lycan ist diese Form der Introspektion als Form eines >inneren Sinns« in jeder Hinsicht vergleichbar mit sinnlichem, nach >Aussen gerichtetem phänomenalem Erleben. ${ }^{462}$

459 Langsam, Harold L.: »Experiences, Thoughts, and Qualia«, Philosophical Studies, [2000] Vol. 99, No. 3, S. 272. Langsam referiert diese Ansicht zwar als eine von Tye wobei er nicht damit einverstanden ist, dass sowohl Gedanken als auch Wahrnehmungserlebnisse bzw. Qualia einen intentionalen Gehalt haben; und auch nicht damit, dass ein Subjekt nur zu intentionalen Gehalten einen bewussten Zugang haben soll. Er geht demgegenüber davon aus, dass wir bei Sinnesqualia nicht-intentionale Gehalte erleben. Vgl. dazu a.a.O., S. 271-272. Langsam gesteht dennoch ein, dass es irgendwie ist, Gedanken zu haben, ihm ist jedoch die phänomenale Differenz beider Arten von Erleben wichtig:»[...] what it is like to have a visual experience of a red object is very different from what it is like to have a thought of a red object«. A.a.O., S. 273.

46o (HOP bedeutet »higher-order perception «). Mehr zu HOP-Theorien vgl. Abschnitt 7.2.3. Vgl. ebenfalls Lycan, William: »Representational Theories of Consciousness «, in: The Stanford Encyclopedia of Philosophy, 2008, Online: http://plato.stanford.edu/archives/ fall2008/entries/consciousness-representational/ [zuletzt geprüft am 28.09.2018], Abschnitt einleitender Erläuterungen.

461 Demzufolge gibt es ohne Introspektion kein qualitatives Erleben, keine Phänomenologie. Vgl. dazu Armstrong, David M.: The nature of mind, Brighton, Sussex: The Harvester Press 1980, S. 63 .

462 Lycan spricht diesbezüglich von einem »internal monitoring«. Vgl. Lycan, William G.: »Consciousness as Internal Monitoring, I: The Third Philosophical Perspectives Lecture«, Philosophical Perspectives, [1995] Vol. 9: AI, Connectionism and Philosophical Psychology, S. 1-14. Vgl. dazu auch Stubenberg, Leopold: Consciousness and qualia, Amsterdam: John Benjamins 1998, S. 79-80. Diese These wird jedoch vor allem von Seiten der HOT-Theorien (»higher-order thought«-Theorien) kritisiert. So gibt es nach Rosenthal diesen >inneren Sinn< nicht und dessen Vergleich mit Sinneswahrnehmung sei müssig. Rosenthal, David M.: »The Independence of Consciousness and Sensory Quality«, in: Consciousness, Villanueva, Enrique [Hrsg.], Boston, Massachusetts: Blackwell 1991, S. 31. Weiter wird kritisiert, dass selbst wenn es einen solchen >inneren Sinn ₹ geben würde, an dieser Konzeption das Problem bestünde, dass dieser eine eigene Phänomenologie haben müsste. Weil es diese spezifische Phänomenologie eines >inneren Sinnes< aber nicht gäbe, könne es auch nicht sein, dass Introspektion eine Form der >inneren< 
Für eine solche Form einer >kognitiven Wahrnehmung`spricht auch Roths Beobachtung, dass es bei einem Erlebnis mit bspw. visueller Modalität keine Rolle spielt, woher der neuronale Reiz kommt. Das heisst, um eine Vorstellung, eine Erinnerung oder einen Gedanken in visueller oder auditiver Sinnesmodalität erleben zu können, ist es nicht notwendig, dass ein visueller Reiz (wie bei einer exterozeptiven Farbwahrnehmung) seinen Ursprung in unserer physischen Umwelt hat. Bei einem Gedanken der Vorstellung oder Erinnerung werden Roth zufolge denn auch die für eine bestimmte Erlebnis-Modalität zuständigen Areale $»$ rein cortexintern ${ }^{463}$ aktiviert:

Alles was im Hinterhauptslappen und unteren Temporallappen passiert, wird vom Gehirn als `Sehen< interpretiert und deshalb von uns in einer bestimmten Weise erlebt, und alles was im oberen Temporallappen passiert, wird als >Hören< erlebt, gleichgültig, woher >eigentlich ‘diese Erregung kommt, ob von einem >natürlichen Input $<[\ldots]$ oder von einer direkten elektrischen Stimulation der Hirnrinde. [...] Wir haben bei Reizung des visuellen Areals $\mathrm{V}_{4}$ Farbempfindungen und bei Reizung des visuellen Areals $\mathrm{V}_{5}$ /MT Bewegungsempfindungen, unabhängig von der >Quelle< dieser Erregung. [...] In der Tat werden ja bei Akten der Vorstellung bzw. Erinnerung von Farbigem oder Bewegtem diese Areale ohne sensorischen Input, d.h. rein cortexintern aktiviert. 464

Die phänomenale Vergleichbarkeit des Erlebens von gedanklichen Vorstellungen und exterozeptiven phänomenalen Wahrnehmungserlebnissen lässt sich des Weiteren dadurch stützen, weil uns auch neuropsychologisch betrachtet $»[\ldots]$ keine absolut verlässlichen Unterscheidungen zwischen [...] $>$ Tatsächlichem< einerseits und $>$ Vorgestelltem $<$ oder $>$ Halluziniertem $<$ andererseits zur Verfügung $[\ldots] \ll^{465}$ stehen..$^{466}$

Wahrnehmung sei. Diese Argumentation ist jedoch wieder insofern angreifbar, dass es fragwürdig ist, ob die Introspektion bzw. der >innere Sinn< für sich genommen zwingend eine eigene Phänomenologie haben muss. Andererseits stellt sich die Frage, ob der, der Introspektion zu Grunde liegende >innere Sinn<, nicht sogar eine eigene Phänomenologie haben könnte. Vgl. dazu auch Stubenberg, Leopold: Consciousness and qualia, Amsterdam: John Benjamins 1998, S. 79-92.

463 Roth, Gerhard: Das Gehirn und seine Wirklichkeit. Kognitive Neurobiologie und ihre philosophischen Konsequenzen, Frankfurt am Main: Suhrkamp 1994, S. 296.

464 Ebd. [Hervorhebung von E. E.].

465 A.a.O., S. 321.

466 Die Unterscheidung zwischen Tatsächlichem und Vorgestelltem ist uns laut Roth nur möglich aufgrund eines »Ausschlussverfahrens«, welches sich in der ontogenetischen Entwicklung sehr spät herausbildet, aber auch bei Erwachsenen nicht absolut verlässlich funktioniert. A.a.O., S. 320. Mit diesem »Ausschlussverfahren« verweist Roth auf die Tatsache, dass alles »[...] in sensorischen corticalen Zentren vom Gehirn als Vorstellung, 
Unter der Berücksichtigung der oben erwähnten These von >kognitiven Wahrnehmungen ${ }^{467}$ und Konzeptionen von Gedanken als Kompositionen aus sinnlichen sowie begrifflichen Anteilen ist es deshalb naheliegend, dass bei nicht-emotiven Gedanken phänomenale Aspekte, bspw. im Sinne phonologisch-verbaler und visueller Vorstellungen, auszuweisen sind. ${ }^{468}$ Wenn wir denken, haben wir nach Tye oft verbale Vorstellungen, die neben den syntaktischen auch phonologische Strukturen unserer Muttersprache aufweisen: »Es ist, als ob wir zu uns selber sprächen. Wir >hören< eine innere Stimme ${ }^{469}$ Bewusste begriffliche Formen des Denkens können nach solchen Konzeptionen demnach mit einem »innere[n] [...] Wort« 470 bzw. inneren Sprechen (»inner speech $\ll),{ }^{471}$ welches wir erleben, verglichen werden. ${ }^{472}$ So besitzen wir nach Merleau-Ponty auch eine »Erfahrung des Denkens«, weil

Erinnerung oder Denken angesehen wird, was nicht aktuelle Wahrnehmung darstellt $[\ldots] \ll$. Ebd.

467 Vgl. z.B. Lycan, William G.: »Consciousness as Internal Monitoring, I: The Third Philosophical Perspectives Lecture«, Philosophical Perspectives, [1995] Vol. 9: AI, Connectionism and Philosophical Psychology, S. 1-14.

468 Vgl. z.B. Chalmers, David J.: »Facing Up to the Problem of Consciousness«, Journal of Consciousness Studies, [1995] Vol. 2, No. 3, S. 202. Vgl. ebenfalls Walter, Sven: »Allgemeine Einleitung: Phänomenales Bewusstsein: Unlösbares Mysterium oder seriöses wissenschaftliches Problem?«, in: Qualia. Ausgewählte Beiträge, Heckmann, Heinz-Dieter / Walter, Sven [Hrsg.], Paderborn: Mentis 20o1, S. 13. Zur Konzeption von Gedanken als eine Form von Vorstellung vgl. auch Damasio, Antonio R.: Descartes' error. Emotion, reason and the human brain, London: Vintage [zuerst 1994]/2006, S. 97, 106, 170 und 180. Über die Ubiquität von Vorstellungen in unserer Kognition vgl. McGinn, Colin: Mindsight. Image, dream, meaning, Cambridge, Massachusetts: Harvard University Press 2006, S. 163.

469 Tye, Michael: »Das Problem primitiver Bewusstseinsformen: Haben Bienen Empfindungen?«, in: Bewusstsein und Repräsentation, Esken, Frank / Heckmann, HeinzDieter [Hrsg.], Paderborn: Mentis [zuerst 1997, engl.]/1999, S. 94. Zur Konzeption von Denken als eine Art >innere Stimmeく vgl. auch Perry, John: »Subjectivity«, in: The Oxford Handbook of Philosophy of Mind, McLaughlin, Brian P. / Beckermann, Ansgar / Walter, Sven [Hrsg.], Oxford: Oxford University Press 2009, S. 226. Vgl. ebenfalls Levine, Joseph: »On Leaving Out What It's Like«, in: The nature of consciousness. Philosophical debates, Block, Ned J. [Hrsg.], Cambridge, Massachusetts: MIT Press 1997, S. 554.

470 Merleau-Ponty, Maurice: Phänomenologie der Wahrnehmung, Berlin (West): De Gruyter [zuerst 1966, franz.]/1974, S. 211.

471 Vocate, Donna R.: »Self-Talk and inner Speech: Understanding the Uniquely Human Aspects of Intrapersonal Communication «, in: Intrapersonal communication. Different voices, different minds, Vocate, Donna R. [Hrsg.], Hillsdale, New Jersey: Erlbaum 20o9, S. 17 .

472 Vocate geht jedoch davon aus, dass es auch (nonverbale) Gedanken gibt, ohne dass mit ihnen gleichzeitig ein »inner speech « vorliegt. Insofern müssen nach ihm Gedanken und »inner speech« nicht zwingend identisch sein. Vgl. a.a.O., S. 17-18. Vocate beschreibt diesbezüglich drei Arten von nonverbalen Gedanken: 1. Das Begehren, etwas zu fragen/verlangen; 2.) den Wunsch, etwas zu übermitteln; 3.) den Wunsch, etwas zu Verstehen. Vgl. a.a.O., S. 17-21. 
wir uns des Denkens nicht nur aufgrund unseres Sprechens bewusst sind, sondern $»[\ldots]$ unser Denken uns geben durch das innere oder das äussere Wort « ${ }^{473}$ Analog zu einem auditiven Wahrnehmungserlebnis in einem Gespräch, weisen phonologisch-verbale Vorstellungen, die das Denken begleiten, ebenfalls phänomenale Gehalte auf. Gedanken bestehen daher nicht nur aus begrifflichen Gehalten im Sinne von Propositionen, ${ }^{474}$ sondern beinhalten auch sinnlich-phänomenale, auditive Anteile. Zudem beschränken sich diese auditiven Vorstellungs-Anteile von Gedanken nicht auf phonologisch-verbale Gehalte, im Sinne eines >inneren Sprechens $\measuredangle{ }^{475}$ Ein Beispiel für einen auditivphänomenalen Anteil eines Gedankens, der jedoch nicht phonologischverbaler Art ist, wäre die Erinnerung an eine bestimmte Melodie (z.B. im Zusammenhang mit dem Gedanken an eine Komponistin). ${ }^{476}$

Für die These, dass Gedanken nicht nur eine auditive Phänomenalität, sondern auch visuelle Vorstellungsgehalte aufweisen können, spricht, dass wir uns bspw. einen Pin-Code nicht zwingend phonologisch-verbal als Zahlbegriff, sondern auch visuell als Ziffer vorstellen und merken können. So berichten Metzinger zufolge Savants, dass sie eine 300-stellige Zahl gewissermassen innerlich »sehen « können. ${ }^{477}$ Auch Damasio betont, dass Gedanken, neben Symbolen und Begriffen, noch auditive und visuelle Vorstellungen aufweisen. Ohne diese sinnlichen Vorstellungs-Anteile wäre es ihm zufolge gar nicht möglich, um einen Gedanken zu wissen:

[...] nobody will deny that thought includes words and arbitrary symbols. But what that statement misses is the fact that both words and arbitrary symbols are based on topographically organized representations and can become images. Most of the words we use in our inner speech, before speaking or writing a sentence, exist as auditory or visual images in our consciousness. If they did not become images $[. .$.$] they would not be anything we could know. { }^{478}$

473 Merleau-Ponty, Maurice: Phänomenologie der Wahrnehmung, Berlin (West): De Gruyter [zuerst 1966, franz.]/1974, S. 211.

474 Vgl. dazu Guttenplan, Samuel [Hrsg.]: A companion to the philosophy of mind, Malden, Massachusetts: Blackwell 2005, S. 487. Vgl. ebenfalls Oppy, Graham: »Propositional attitudes«, 1998, Online:https://www.rep.routledge.com/articles/thematic/propositionalattitudes/v-1 [zuletzt geprüft am 25.09.2018].

475 Vgl. Perry, John: "Subjectivity«, in: The Oxford Handbook of Philosophy of Mind, McLaughlin, Brian P. / Beckermann, Ansgar / Walter, Sven [Hrsg.], Oxford: Oxford University Press 2009, S. 226.

476 Vgl. dazu auch Stubenberg, Leopold: Consciousness and qualia, Amsterdam: John Benjamins 1998, S. 293.

477 Vgl. Metzinger, Thomas: Subjekt und Selbstmodell. Die Perspektivität phänomenalen Bewusstseins vor dem Hintergrund einer naturalistischen Theorie mentaler Repräsentation, Paderborn: Mentis 1999, S. 85 .

478 Damasio, Antonio R.: Descartes' error. Emotion, reason and the human brain, London: Vintage [zuerst 1994]/2006, S. 106. 
Tye unterscheidet demgegenüber Gedanken, die in Verbindung mit phonologisch-verbalen Vorstellungen stehen, von solchen, die mit visuellem und vorsprachlichem Wiedererkennen zusammenhängen. Auch wenn sich dies von Damasios Gedankenkonzeption unterscheidet, so weist dies dennoch darauf hin, dass der phänomenale Gehalt von Gedanken aus verschiedenen Komponenten besteht. ${ }^{479}$ Auch die somatosensorische Modalität phänomenalen Erlebens kann Teil eines Gedankens sein: Wenn wir bspw. an den Charakter einer Schmerzempfindung beim Zahnarzt denken und deshalb überzeugt sind, dass Zahnarztbesuche mit Schmerzen verbunden sind, ist es plausibel, dass mit diesem Gedanken bzw. Urteil phänomenales Bewusstsein einhergeht. ${ }^{480}$ McGinn spricht ebenfalls von der Kompositionalität von Vorstellungen, die er als aus sinnlichen und begrifflichen Anteilen zusammengesetzt versteht, während Chalmers auf die reichhaltige Phänomenologie (»rich phenomenology «) ${ }^{481}$ von visuellen, auditiven, taktilen, olfaktorischen und gustatorischen Vorstellungen verweist. ${ }^{482}$

Die Konzeption, dass Gedanken, weil sie auch sinnliche (Vorstellungs-) Anteile haben, nur schon deshalb phänomenale Aspekte aufweisen müssen, ist vom herkömmlichen Qualia-Verständnis, genau betrachtet noch gar nicht so weit entfernt. Es ist aber nicht nur irgendwie, beim Denken gleichsam eine »innere Stimme « ${ }^{483} \mathrm{zu} \gg$ hören

479 Vgl. dazu Tye, Michael: »Das Problem primitiver Bewusstseinsformen: Haben Bienen Empfindungen?«, in: Bewusstsein und Repräsentation, Esken, Frank / Heckmann, HeinzDieter [Hrsg.], Paderborn: Mentis [zuerst 1997, engl.]/1999, S. 94. Über visuelle bzw. phonologische Arten des Denkens, vgl. auch Baars, Bernard J.: Das Schauspiel des Denkens. Neurowissenschaftliche Erkundungen, Stuttgart: Klett-Cotta 1998, S. 74 und 110.

48 o Vgl. dazu auch Soldati, Gianfranco: »Begriffliche Qualia. Zur Phänomenologie der Bedeutung «, in: Anatomie der Subjektivität. Bewusstsein, Selbstbewusstsein und Selbstgefühl, Grundmann, Thomas / Hofmann, Frank / Misselhorn, Catrin et al. [Hrsg.], Frankfurt am Main: Suhrkamp 2005, S. 159.

481 Chalmers, David J.: The conscious mind. In search of a fundamental theory, New York: Oxford University Press 1996, S. 9.

482 Vgl. auch McGinn, Colin: Mindsight. Image, dream, meaning, Cambridge, Massachusetts: Harvard University Press 2006, S. 163. Über imaginative Sinnes-Qualia vgl. auch Graham, George / Horgan, Terence / Tienson, John: »Phenomenology, Intentionality, and the Unity of the Mind«, in: The Oxford Handbook of Philosophy of Mind, McLaughlin, Brian P. I Beckermann, Ansgar / Walter, Sven [Hrsg.], Oxford: Oxford University Press 2009, S. 516. Zum Zusammenhang von Vorstellungen und Gedankenprozessen vgl. auch Damasio, Antonio R.: The feeling of what happens. Body, emotion and the making of consciousness, London: Vintage 2000, S. 318.

483 Tye, Michael: »Das Problem primitiver Bewusstseinsformen: Haben Bienen Empfindungen?«, in: Bewusstsein und Repräsentation, Esken, Frank / Heckmann, HeinzDieter [Hrsg.], Paderborn: Mentis [zuerst 1997, engl.]/1999, S. 94. 
visuelle oder auditive Vorstellungen zu erleben, ${ }^{484}$ sondern es ist auch irgendwie, einen Gedanken zu verstehen. Soldati untermauert dies mit einem Gedankenexperiment von Strawson: Jack, der nur Englisch versteht und Jacques, der nur Französisch versteht, hören zusammen französische Nachrichten im Radio. Lässt sich nun nicht feststellen, dass Jacques, der freilich die Nachrichten nicht nur hört, sondern auch versteht, ein anderes Gesamterlebnis hat, als Jack, der zwar aus dem Radio dieselben Laute hört, diese aber nicht versteht? Das Erleben von Jack beschränkt sich, auf ein Hören von für ihn sinnlosen Wortfolgen, von denen er aus der Erfahrung vielleicht sagen kann, dass sie nach Französisch klingen. Jacques dagegen erlebt nicht einfach nur sinnlose Wortfolgen, sondern zusammen mit dem Hörerlebnis, erlebt er diese Wortfolgen nicht nur als akustische Laute, sondern als sinnvoll bzw. als gehaltvoll. Wenn dem so ist, dann müsste es aber eine Art >Erlebnis des Verstehens< geben, welches sich vom Erleben des Hörens von unverstandenen und deshalb sinnfreien Wortfolgen unterscheidet. ${ }^{485}$ Das bedeutet schliesslich, dass es sowohl irgendwie ist, wenn wir einen Satz verstehen als auch, wenn wir einen Satz nicht verstehen. Insofern kann man, neben den phänomenalen Aspekten gedanklich-sinnlicher Vorstellungsgehalte, auch dem Verstehen von Gedanken eine phänomenale Qualität zusprechen. Aus solchen Überlegungen folgert schliesslich Soldati, dass Verstehen eine phänomenale Qualität besitzt, weshalb er »begriffliche Qualia «486 postuliert. Diese »begrifflichen Qualia« seien jedoch von sensorischen Vorstellungsgehalten, die Soldati zufolge Gedanken begleiten, zu unterscheiden. ${ }^{487}$

Im Zusammenhang mit seiner Konzeption »begrifflicher Qualia « ${ }^{488}$ geht Soldati davon aus, dass auch (motivierte) Überzeugungen wie Urteile von einer »bewusst erlebte[n] phänomenale[n] Komponente « 489 begleitet werden, denn das Erfassen von spezifischen Bedeutungen wird bewusst erlebt. In diesem Fall handelt es sich also um das bewusste Erfassen der Bedeutung einer Überzeugung. ${ }^{490}$ Für Strawson hat sowohl bewusstes Denken als auch

$484 \mathrm{Zu}$ auditiv strukturierten Vorstellungen im Sinne von Qualia vgl. auch Stubenberg, Leopold: Consciousness and qualia, Amsterdam: John Benjamins 1998, S. 293.

485 Vgl. Soldati, Gianfranco: »Begriffliche Qualia. Zur Phänomenologie der Bedeutung «, in: Anatomie der Subjektivität. Bewusstsein, Selbstbewusstsein und Selbstgefühl, Grundmann, Thomas / Hofmann, Frank / Misselhorn, Catrin et al. [Hrsg.], Frankfurt am Main: Suhrkamp 2005, S. 145. Vgl. dazu auch a.a.O., S. 146-147 und 149.

486 A.a.O., S. 148.

487 Vgl. a.a.O., S. 145 und 161.

488 A.a.O., S. 148.

489 A.a.O., S. 147.

49 S Soldati meint jedoch, dass nur einige propositionale Einstellungen einen spezifischen phänomenalen Charakter haben. Vgl. ebd. Zur Konzeption der Phänomenalität von 
Verstehen den Charakter qualitativen Erlebens. Die Phänomenalität des Verstehens ist für ihn ebenfalls unabhängig von denjenigen vorgestellten Sinnesmodalitäten, die einen begleitenden Teil von Gedanken ausmachen können:

When one thinks consciously [...] apprehension of conceptual content occurs and is part of the course and content of one's experience [...]. This may be clearer to insomniacs, who spend long hours thinking in the dark, than to instant sleepers. [...] My central claim is that the apprehension and understanding of cognitive content, considered just as such and independently of any accompaniments in any of the sensory-modality based modes of imagination or mental representation, is part of experience, part of the flesh or content of experience, and hence, trivially, part of the qualitative character of experience. ${ }^{491}$

So können wir mit Strawson und Soldati feststellen, dass es auch irgendwie ist, zu verstehen. ${ }^{492}$ Verstehen, auch wenn es keine ostentative oder dramatische phänomenale Qualität besitzt, kann als eine Form des Erlebens, als >VerstehensErlebnis $(»$ understanding-experience $\ll),{ }^{493}$ verstanden werden, auch wenn dies natürlich nicht dem herkömmlichen, engen Verständnis von Qualia entspricht:

$[\ldots]$ there is understanding-experience, understanding-experience whose very existence is sometimes doubted, perhaps because it has no obvious experiential character that it can call its own. It has no striking experiential feel in the way experience in any of the sensory modalities usually does. But this does not show that there is nothing that can be correctly called sunderstanding-experiences. Rather, it shows that in certain contexts of discussion, we may still be inclined to appeal to an excessively restricted notion of what experience is. ${ }^{494}$

Fazit: Die hier dargelegten Beispiele und Überlegungen, zeigen auf, dass Gedanken, selbst wenn sie nicht-emotiver Art sind, ebenfalls phänomenale Aspekte aufweisen, ja dass es sogar von einem »extrem engen « ${ }^{495}$ QualiaVerständnis herrührt, wenn wir nur nicht-begrifflichen, sensorischen mentalen Zuständen Phänomenalität zusprechen.

Überzeugungen und propositionalen Einstellungen vgl. auch Flanagan, Owen J.: Consciousness reconsidered, Cambridge, Massachusetts: MIT Press 1992, S. 69.

491 Strawson, Galen: Mental reality, Cambridge, Massachusetts: MIT Press 1994, S. 12.

492 Zur Konzeption, dass Verstehen eine phänomenale Qualität hat, vgl. auch Jung, Matthias / Heilinger, Jan-Christoph [Hrsg.]: Funktionen des Erlebens. Neue Perspektiven des qualitativen Bewusstseins. Band 5: Humanprojekt, Berlin: De Gruyter 2009, S. 2.

493 Strawson, Galen: Mental reality, Cambridge, Massachusetts: MIT Press 1994, S. 8.

494 Ebd. [Hervorhebung von E. E.].

495 Ebd. 
Grundsätzlich kann man festhalten, dass sich, grob vereinfacht, zwei Kategorien von phänomenalen Eigenschaften bei nicht-emotiven Formen des Denkens ausweisen lassen: a.) phänomenale Eigenschaften von Vorstellungserlebnissen im Sinne herkömmlicher, aber imaginierter Sinnes-Qualia; ${ }^{496}$ b.) eine phänomenale Eigenschaft sui generis, ${ }^{497}$ die ganz allgemein darin besteht, dass es irgendwie ist, sich als denkend, verstehend, urteilend zu erleben. Ein phänomenal adäquater Qualia-Begriff ist deshalb, im Zusammenhang mit dem was wir >Denken $<$ nennen, auch um den Bereich phonologisch-verbaler bzw. generell auditiver, visueller, taktiler, olfaktorischer, gustatorischer und somatosensorischer ${ }^{498}$ Vorstellungserlebnisse sowie um Erlebniszustände des Verstehens, des Erfassens von Überzeugungen oder, ganz allgemein, des >sichals-denkend-Erlebens $<$ zu erweitern. Die hier aufgezählten Formen von Qualia bei nicht-emotiven Gedanken werde ich als D-Qualia bezeichnen. ${ }^{499}$

496 Vgl. dazu auch Pitt, David: »The Phenomenology of Cognition or what is it Like to Think that P?«, Philosophy and Phenomenological Research, [2004] Vol. 69, No. 1, S. 3-4.

497 Vgl. dazu a.a.O., S. 4, 10 und 24.

498 Z.B. Schmerz bei der oben genannten Vorstellung eines Zahnarztbesuches.

499 Weitere Hinweise zu gedanklichen Qualia finden sich bei Horgan, Terence / Tienson, John: »The Intentionality of Phenomenology and the Phenomenology of Intentionality«, in: Philosophy of mind. Classical and contemporary readings, Chalmers, David J. [Hrsg.], New York: Oxford University Press 2002, S. 520-533. Oder: Flanagan, Owen J.: Consciousness reconsidered, Cambridge, Massachusetts: MIT Press 1992, S. 62-68. Oder: Stich, Stephen: »Beliefs and Subdoxastic States«, Philosophy of Science, [1978] Vol. 45, No. 4, S. 504. Oder: Block, Ned J.: »Consciousness «, in: A companion to the philosophy of mind, Guttenplan, Samuel [Hrsg.], Malden, Massachusetts: Blackwell 2005, S. 211. 